\title{
Organic Single-Crystal Field-Effect Transistors
}





\section{Organic Single-Crystal Field-Effect Transistors}

\section{Proefschrift}

ter verkrijging van de graad van doctor aan de Technische Universiteit Delft,

op gezag van de Rector Magnificus Prof. dr. ir. J. T. Fokkema, voorzitter van het College voor Promoties,

in het openbaar te verdedigen op vrijdag 28 oktober 2005 om 10.30 uur

door

\section{Ruth Wilhelmine Ireen DE BOER}

natuurkundig ingenieur

geboren te Roermond. 
Dit proefschrift is goedgekeurd door de promotor:

Prof. dr. ir. T. M. Klapwijk

Toegevoegd promotor:

Dr. A. F. Morpurgo

Samenstelling promotiecommissie:

Rector Magnificus voorzitter

Prof. dr. ir. T. M. Klapwijk Technische Universiteit Delft, promotor

Dr. A. F. Morpurgo

Technische Universiteit Delft, toegevoegd promotor

Prof. dr. M. E. Gershenson Rutgers University

Prof. dr. D. van der Marel University of Geneva

Prof. dr. M. Orrit Universiteit Leiden

Prof. dr. T. T. M. Palstra Rijksuniversiteit Groningen

Prof. dr. L. D. A. Siebbeles Technische Universiteit Delft

Prof. dr. A. Schmidt-Ott Technische Universiteit Delft, reservelid

\section{T̛UDelft \&OM}

Cover design: R. W. I. de Boer

Front: Chemical model of a rubrene molecule. The background is an optical microscope picture of a rubrene single-crystal field-effect transistor. In reality, the area shown in the microscope picture is approximately $1.2 \times 0.8 \mathrm{~mm}^{2}$. The rubrene crystal (red rectangular area) was manually aligned with the underlying prefabricated substrate.

Back: Optical microscope picture (using crossed polarizers) of tetracene polycrystalline structure grown from chlorobenzene solution.

Printed by: Ponsen \& Looijen

Copyright (C) 2005 by R. W. I. de Boer

Casimir PhD Series, Delft-Leiden 2005-05

ISBN 90-8593-005-7 
Why seek to scale Mount Everest Queen of the Air

Why strive to crown that cruel crest And deathward dare?

Said Mallory of dauntless quest 'Because it's there.'

\section{Robert William Service}




\section{Contents}

1 Introduction $\quad 1$

1.1 Organic electronics . . . . . . . . . . . . . . . 2

1.2 Organic single-crystal FETs for charge transport studies . . . . . 4

1.3 Outline of this Thesis . . . . . . . . . . . . . 8

References ........................ 9

2 Physics of organic semiconductors 13

2.1 Introduction . . . . . . . . . . . . . . . . . . . 14

2.2 Organic molecules . . . . . . . . . . . . . . . . . . . . 15

2.3 Intermolecular organization . . . . . . . . . . . . 18

2.3.1 Intermolecular interactions . . . . . . . . . . . . . . 18

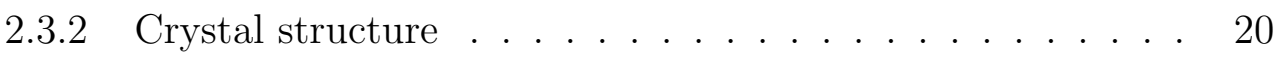

2.4 Charge transport mechanism in $\pi$ systems . . . . . . . . . . . . . 23

2.4.1 Intrinsic mobility . . . . . . . . . . . . . . . . . . . . . . . . . . . . . .

2.4.2 Polaron transport . . . . . . . . . . . . . . . . . . 26

2.4.3 Localization by defects . . . . . . . . . . . . . . . . 29

2.5 Principles of charge transport experiments . . . . . . . . . . 30

2.5.1 Charge injection into organic semiconductors . . . . . . . 30

2.5.2 Field-effect transistors . . . . . . . . . . . . . . . . . 33

References .................. . . 37

3 From organic molecule to single-crystal transistor 41

3.1 Single-crystal growth and characterization . . . . . . . . . . . 42

3.1.1 Single-crystal growth . . . . . . . . . . . . . . . . . . . . . . . . . . . . . . . . 42

3.1.2 Crystal characterization . . . . . . . . . . . . . 43

3.2 Single-crystal FET fabrication techniques . . . . . . . . . . . . . . . . . 45

3.2.1 Electrostatic bonding technique . . . . . . . . . . 46

3.2.2 "Direct" FET fabrication on the crystal surface . . . . . . 49

References . . . . . . . . . . . . . . 51 
4 Space-charge limited transport and time-of-flight measurements in tetracene single crystals $\quad 53$

4.1 Introduction . . . . . . . . . . . . . . . . . . 54

4.2 Crystal growth and sample preparation . . . . . . . . . . 55

4.3 DC transport through tetracene single crystals . . . . . . . . . . 56

4.3.1 Estimate of the carrier mobility . . . . . . . . . . 56

4.3.2 Measurements of $I-V$ characteristics . . . . . . . . . 57

4.3.3 Deep traps in the bulk and at the contacts . . . . . . . . 60

4.4 TOF experiments on tetracene single crystals . . . . . . . . . 62

4.4.1 Technical aspects of TOF experiments . . . . . . . . . 62

4.4.2 Measurement of TOF transients . . . . . . . . . . . . 63

4.5 Summary . . . . . . . . . . . . . . . . . 65

References ...................... 67

5 Influence of surface traps on space-charge limited current $\quad 69$

5.1 Introduction . . . . . . . . . . . . . . . . . 70

5.2 Modifications to the conventional SCLC theory . . . . . . . . 71

5.3 Manifestations of surface traps in calculated $I-V$ curves . . . . . 73

5.3.1 Asymmetric $I-V$ characteristics . . . . . . . . . . . . 73

5.3.2 Additional manifestations of surface traps . . . . . . . . 75

5.3.3 Perturbed electrostatic profile . . . . . . . . . . . 77

5.4 Conclusion . . . . . . . . . . . . . . . . . . 78

References ....................... 78

6 Field-effect transistors on tetracene single crystals 81

6.1 Introduction . . . . . . . . . . . . . . . . . . . 82

6.2 Electrostatic adhesion of thin crystals . . . . . . . . . . . 82

6.3 Characterization of FET performance . . . . . . . . . . . . 84

6.4 Non-monotonous temperature dependence of mobility . . . . . . . 86

6.5 Comparison with TOF experiments . . . . . . . . . . . . . 87

6.6 Conclusion . . . . . . . . . . . . . . . . . . . 87

References . . . . . . . . . . . . . . . 87

7 Ambipolar $\mathrm{Cu}$ - and Fe-phthalocyanine single-crystal field-effect $\begin{array}{lr}\text { transistors } & 91\end{array}$

7.1 Introduction . . . . . . . . . . . . . . . . . . . 92

7.2 Processing-related specifications of $\mathrm{CuPc}$ and $\mathrm{FePc}$ crystals . . . . 92

7.3 Observations of ambipolar transport . . . . . . . . . . . . . 93

7.4 Intrinsic and extrinsic effects of high work-function Au contacts . 94 
7.5 Opportunities for further research . . . . . . . . . . . . . . 97

References . . . . . . . . . . . . . . . 97

8 Influence of the gate leakage current on the stability of organic single-crystal field-effect transistors 101

8.1 Introduction . . . . . . . . . . . . . . . . . . . . . 102

8.2 Characterization of sputtered $\mathrm{Ta}_{2} \mathrm{O}_{5}$ and $\mathrm{ZrO}_{2}$ films . . . . . . 102

8.3 Anomalous electrical behavior of $\mathrm{Ta}_{2} \mathrm{O}_{5}$ and $\mathrm{ZrO}_{2}$ FETs . . . . . 103

8.4 Performance degradation due to small gate-leakage currents . . . 104

8.5 Conclusion . . . . . . . . . . . . . . . . . 107

References . . . . . . . . . . . . . . . . 108

9 Influence of the gate dielectric and charge density on the performance of rubrene single-crystal field-effect transistors 109

9.1 Introduction . . . . . . . . . . . . . . . . . . 110

9.2 Influence of the gate dielectric on rubrene FET mobility . . . . 110

9.2.1 Technical aspects . . . . . . . . . . . . . . 111

9.2.2 Experimentally observed influence of gate dielectric . . . . 112

9.2.3 Proposed mechanism of polaronic dressing . . . . . . . . . 115

9.3 High charge density study on rubrene FETs . . . . . . . . . . . . 116

9.3.1 Unconventional FET characteristics . . . . . . . . . . . . 117

9.3.2 Discussion of explanatory scenarios . . . . . . . . . . . 119

References . . . . . . . . . . . . . . . . . 123

$\begin{array}{ll}\text { Summary } & 125\end{array}$

$\begin{array}{lr}\text { Samenvatting } & 129\end{array}$

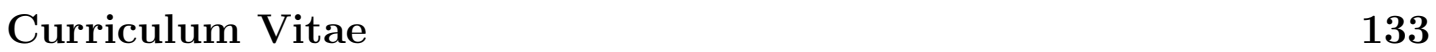

$\begin{array}{ll}\text { List of publications } & 135\end{array}$

$\begin{array}{lr}\text { Acknowledgements } & 137\end{array}$ 
Chapter 1

\section{Introduction}




\subsection{Organic electronics}

It is hard to imagine our modern world without plastic. Even more amazing is the fact that the first completely synthetic plastic, bakelite, was developed only 100 years ago. Since the 1950s, plastics have grown into a major industry that affects all of our lives - from providing improved packaging to giving us new textiles, to permitting the production of wondrous new products and cutting edge technologies in such things as televisions, cars and computers. Plastics even allow doctors to replace worn-out body parts, enabling people to live more productive and longer lives. In fact, since 1976, plastic has been the most used material in the world and was voted one of the greatest innovations of the millennium [1].

Plastics possess a number of profitable qualities that make them very appealing for applications. Their ease of low-temperature processing is of crucial interest for large-scale commercialization. Most plastics are polymers that belong to the class of organic materials [2], which entails a large variety of compounds that can be chemically designed and synthesized. Compared to wood, steel, glass, and many other substances, organic materials are typically light-weighted and cheap. Moreover, they link these features with beneficial mechanical properties, like strength and flexibility.

Since 1977, when for the first time electrical conduction in organic polymers was reported by Heeger, MacDiarmid and Shirakawa [3, 4], the electrical properties of organic materials have received a growing amount of attention. Before that time, polymers were known as insulators only. Heeger, MacDiarmid and Shirakawa found that, upon chemical doping with halogens, the electrical conductivity of polyacetylene could be increased over many orders of magnitude, from an insulating state to a metallic state. Conducting polymers offered the promise of achieving a new generation of polymers: materials that exhibit the electrical and optical properties of metals or semiconductors and which retain the attractive mechanical properties and processing advantages of polymers [5].

This combination of properties has aroused the idea of developing organic electronics: organic semiconductors as the basis for electronic components, like diodes, transistors, rectifiers, solar cells, photo-detectors, electro-optic switches and sensors. Electronics based on organic semiconductors is by no means expected to replace silicon in high performance, but instead is aimed at complementary applications where ease of fabrication, mechanical flexibility, low-cost and/or large-area applicability are of particular importance [6, 7]. For example, plastic circuitry is anticipated for use as memory elements in transaction cards and identification tags, and as display drivers, in portable computers and electronic paper. Already a few years ago, in 2002, the first commercial application 


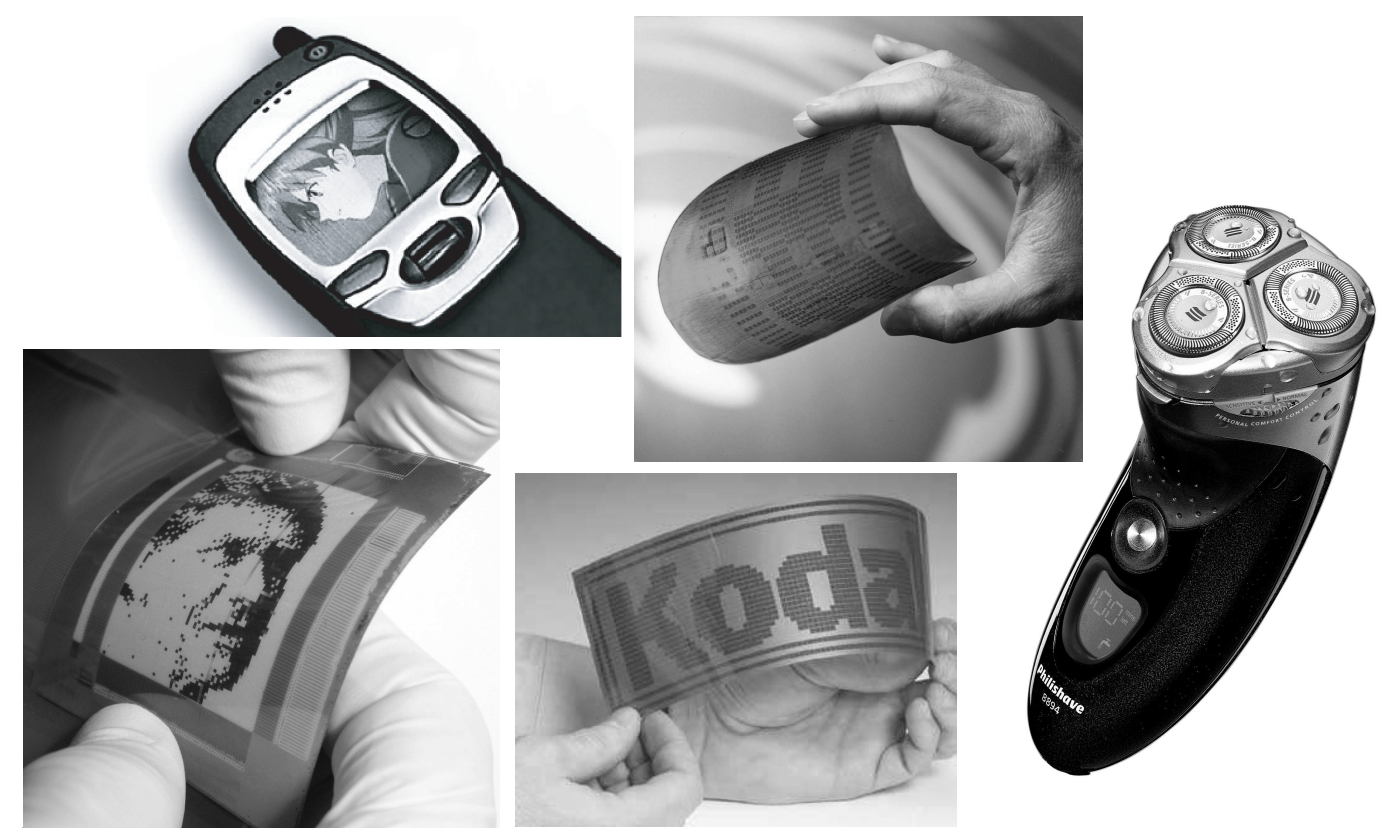

Figure 1.1: Prototypes and commercial applications of organic electronics as (flexible) displays. Acknowledgement of source, going clockwise from left upper corner: Cambridge Display Technologies, Philips Research $(2 \times)$, Kodak R\&D and Plastic Logic.

of polymer electronics was brought on the consumer market: Philips Research employed a polymer LED segment display in the Philishave 8894, better known as the "James Bond" shaver.

At present, the selection of organic semiconductors is much broader than polymers only. The use of small organic molecules (e.g. $\mathrm{C}_{60}$, pentacene and thiophenes [8]) offers the advantage of a higher degree of structural ordering and, related to that, a better device performance, measured by the value of the charge carrier mobility. Mobilities comparable to that of amorphous silicon are now within reach, but the commercial applicability of organic semiconductors has stimulated the quest for the ultimate electrical performance limits of organic compounds, and the urge to identify the aspects that influence this performance. This requires a fundamental understanding of charge injection and transport, that can only be achieved with innovative experimental methods and theoretical investigations. Therefore, organic materials are subject to not only industrial development, but also scientific research.

For the fundamental understanding of organic semiconductor physics, knowledge of the thoroughly studied inorganic semiconductors does not suffice. The electronic properties of van der Waals bonded organic semiconductors are pro- 
foundly different from those of covalently/ionically-bonded inorganic semiconductors $[9,10]$. In particular, the single-electron approach that is generally used for describing charge carrier transport in inorganic materials, is not successful here. Organic semiconductors have a highly-polarizable crystal lattice, which gives rise to a strong coupling of the charge carrier to its crystal environment. Each charge carrier is dressed with a cloud of induced polarizations, resulting in a self-trapped electronic state, i.e. the polaron. The polaronic effects play a key role in determining transport in organic materials, as they "shape" both the DC transport and optical properties of these materials.

Thus, additional to the stimulus originating from applications, research on organic matter broadens the fundamental understanding of solid-state physics. However, because of a very complicated character of the many-particle interactions involved in polaron formation, this problem has been treated mainly at the phenomenological level (see Chapter 7 in Ref. [9]). Many basic aspects of this problem have not been addressed yet, and a well-developed microscopic description of the charge transport in organic materials is still lacking. For that purpose, experiments are necessary that explore further, and in more detail, the aspects of intrinsic transport behavior of organic semiconductors.

\subsection{Organic single-crystal FETs for charge trans- port studies}

Until recently, the experimental study of the low-frequency intrinsic electronic properties of organic semiconductors have been performed only on bulk ultra-pure crystals $[11,12]$. In the Time-of-Flight (TOF) experiments, shown in Fig. 1.2, by the group of Norbert Karl at Stuttgart University [13, 14], it has been found that the mobility of non-equilibrium carriers generated by light absorption in ultrahigh-purity oligomeric crystals can be as high as $400 \mathrm{~cm}^{2} / \mathrm{Vs}$ at low temperatures (the latter mobility value is comparable to the mobility of electrons in silicon MOSFETs at room temperature). This behavior suggests that coherent, bandlike polaronic transport is possible in crystals of small organic molecules.

To further investigate the electronic properties of organic materials, it is important to go beyond the TOF measurements. One of the alternative techniques to probe the charge transport on a semiconductor surface is based on the electric field effect [15]. Continuous tuning of the charge density induced by the transverse electric field enables the systematic study of charge transport, in particular the regime of large carrier density that cannot be accessed in the TOF experiments. The field effect forms the basis for operation of silicon field-effect transistors 


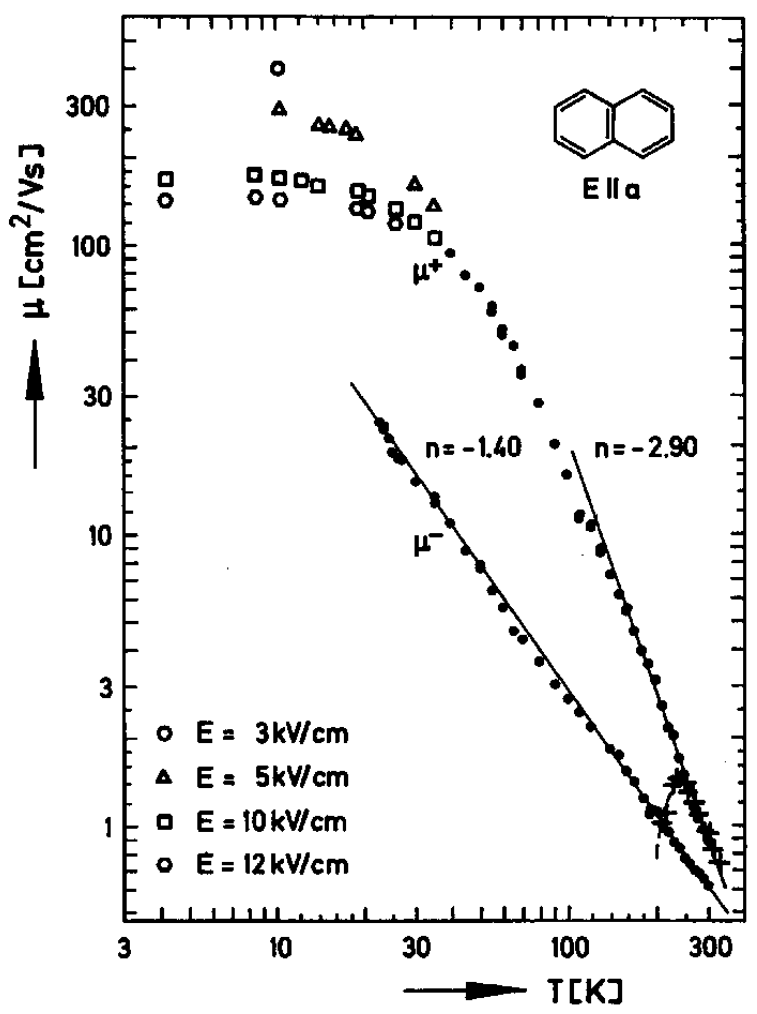

Figure 1.2: Electron and hole mobility $\mu$ versus temperature $T$ in ultra-pure single crystals of naphthalene, as measured in Time-of-Flight (TOF) experiments. The solid lines indicate a $T^{n}$ power-law temperature dependence with exponents $n$ as indicated in the figure. For holes, mobility values as high as $400 \mathrm{~cm}^{2} / \mathrm{Vs}$ are observed at low temperature [13].

(FETs), the workhorses of modern inorganic electronics. The field-effect technique is also used in fundamental studies as a method to control the behavior of strongly correlated electron systems such as high-temperature superconductors (see, e.g. [16]) and colossal magnetoresistance manganites [17]. Other recent examples of applications of this remarkably simple and very successful principle are the electric-field tuning of the metal-insulator transition in cuprates [18] and vanadium oxides [19], the electrostatic control of ferromagnetism in Mn-doped GaAs [20].

Organic semiconductors are, in principle, well suited for the field-effect experiments. Owing to the weak van der Waals bonding, the surface of organic semiconductors (e.g. polyacenes [21, 22] and conjugated polymers [23]) is characterized by an intrinsically low density of dangling bonds that can act as the 
charge traps, and, hence, by a low threshold for the field effect. This fact is at the origin of the rapid progress of organic field-effect transistors based on thin-film technology, i.e. organic Thin-Film Transistors (TFTs) [7, 24, 25].

Unfortunately, thin-film transistors are not suitable for the study of intrinsic electronic properties of organic conductors, because their characteristics are often strongly affected by imperfections of the film structure and by insufficient purity of organic materials (see, e.g., Fig. 1.3 and [7, 26, 27]). As a consequence, these devices commonly exhibit an exponential decrease of the mobility of field-induced charge carriers with lowering temperature [28]. This behavior contrasts sharply to a rapid increase of the mobility with decreasing temperature, observed in the TOF experiments with bulk ultra-pure organic crystals $[13,14]$. Because of a very strong dependence of the organic TFT parameters on fabrication conditions, some researchers came to a pessimistic conclusion that even the best organic TFTs "may not be appropriate vehicles for illuminating basic transport mechanisms in organic materials" [29].

To explore the intrinsic electronic properties of organic materials and the physical limitations on the performance of Organic FETs (OFETs), devices based on single crystals of organic semiconductors are needed, similar to the singlecrystal structures of inorganic electronics. One of the major impediments to realization of the single-crystal OFETs is the lack of hetero-epitaxial growth technique for the van der Waals bonded organic films. In this situation, the only viable option to study the intrinsic charge transport on the surface of organic semiconductors is to fabricate the field-effect structures on the surface of free-standing organic molecular crystals (OMCs). However, fabrication of singlecrystal OFETs poses a technological challenge. Because the surface of OMCs can be damaged much more easily than that of their inorganic counterparts, organic materials are by and large incompatible with conventional microelectronic processing techniques such as sputtering, photolithography, etc. This is why the systematic investigation of single-crystal OFETs has started only very recently $[30,31,32,33,34,35]$, after the successful development of a number of novel fabrication schemes (for earlier work see [36, 37]).

Realization of the single-crystal OFETs opens a new avenue for the study of charge transport in highly ordered molecular systems. The use of single-crystal OFETs as an experimental tool enables the investigation of aspects of charge transport in organic materials that could not be addressed in the TOF experiments. One of the important distinctions between these two types of experiments is the magnitude of carrier densities. Very low densities of charge carriers in the TOF experiments make interactions between them insignificant. At the same time, in the field-effect experiments with organic materials, where accumulation 


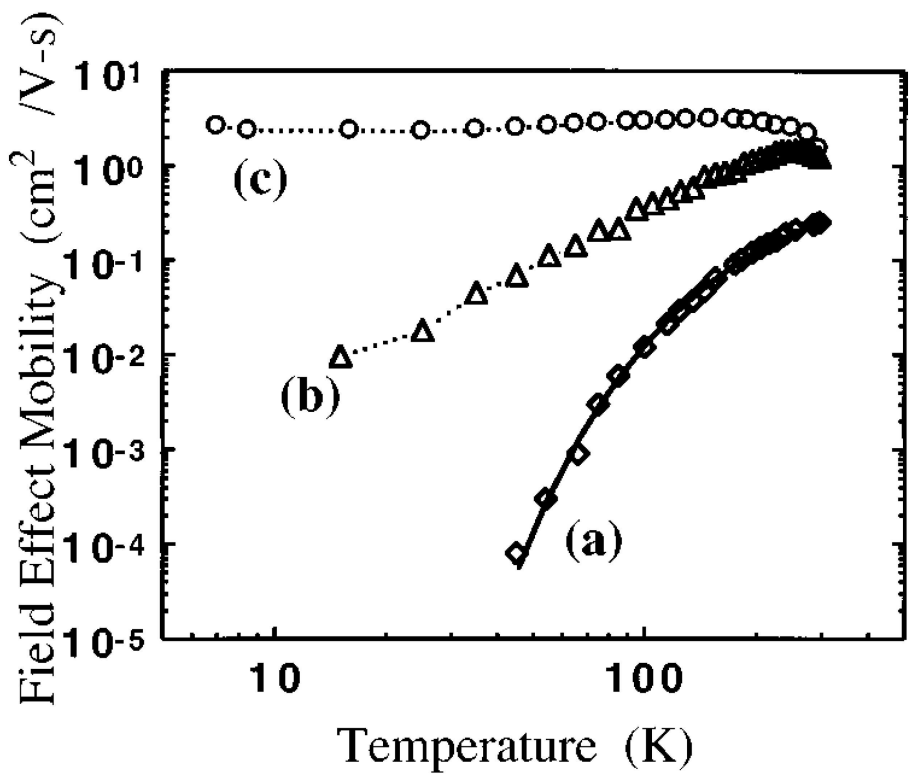

Figure 1.3: Field-effect mobility versus temperature for three different pentacene thin-film transistors grown under similar conditions [29]. These measurements are among the best results that have been obtained for organic thin-film transistors, which commonly exhibit an exponential decrease of the mobility with lowering temperature (in contrast with the power-law dependence shown in Fig. 1.2). Moreover, the three diverse curves ( $\mathrm{a}, \mathrm{b}$ and $\mathrm{c}$ ) illustrate the irreproducibility that is typical for organic thin-film-transistors.

of $\sim 1$ carrier per molecule is feasible with the use of high- $\epsilon$ dielectrics, these interactions could play a major role. Indeed, it is well-known that at a sufficiently high density of chemically-induced carriers, the potassium-doped fullerene $\mathrm{K}_{\mathrm{x}} \mathrm{C}_{60}$ exhibits superconductivity $(\mathrm{x}=3)$ and a Mott-Hubbard insulating state $(\mathrm{x}=4)$ [38]. This example illustrates a great potential of experiments with the singlecrystal OFETs.

The first working FET on the surface of a free-standing organic molecular crystal has been fabricated three years ago [30]. Though this field is in its infancy, the progress has been remarkably rapid, with new record values of carrier mobility for OFETs being achieved, new promising organic materials being introduced, and new device processing techniques being developed. In this Thesis, we present our contribution to this progress.

When this work started four years ago, organic single-crystal FETs were not yet developed. We started with the growth and characterization of high-quality organic single crystals that are needed for the fabrication of a high-quality organic FET. However, the vulnerable crystals are easily damaged during handling 
and processing, and therefore, a crucial step in this work was the development of a non-damaging fabrication technique for organic single-crystals FETs. The success of our electrostatic bonding method is indicated by observed signatures of intrinsic transport, like high charge carrier mobilities, an increase of the mobility at lowering temperature and ambipolar transport. A high level of reproducibility characterizes the transport measurements, as well as good agreement with data obtained on single-crystal OFETs fabricated with different methods. Accordingly, the electrostatic bonding technique to a prefabricated silicon substrate is currently one of the three established high-quality OFET assembly techniques (see Chapter 3). An important advantage of the method is its compatibility with different types of gate insulators. In combination with the high-quality and the reproducibility of the experiments, it allows to identify aspects that influence charge transport and to investigate a new operation regime, i.e. the high charge density regime, of organic single-crystal FETs.

\subsection{Outline of this Thesis}

The outline of this Thesis is as follows. Chapter 2 discusses the electronic structure in organic semiconductors, from electrons and holes in single molecules to charge transport properties in organic single-crystal field-effect transistors. The last Section of this Chapter focuses on standard FET characteristics and theory, based on inorganic semiconductors, with which the organic FET experimental results in later Chapters are compared.

Chapter 3 also walks the road from organic molecule to single-crystal transistor, but then from the viewpoint of fabrication. It describes the Physical Vapor Transport (PVT) growth of high-quality organic single crystals, and their characterization. The existing techniques to process the crystals into single-crystal FETs are explained with their advantages and disadvantages.

The single-crystal characterization is further treated in Chapter 4 . Timeof-Flight (TOF) and Space-Charge Limited Current (SCLC) measurements on tetracene single crystals, grown in our PVT setup, show that signatures of intrinsic electronic transport are visible, which demonstrates the high quality of the crystals. Also, it is concluded that the fabrication of the electrical contacts on the organic crystals is a delicate process that can affect the SCLC results considerably. In relation to this, the following Chapter 5 deepens the understanding of SCLC theory, by studying the effect on SCLC transport of surface traps at the crystal/contact interface.

The technique applied more frequently in this Thesis for fabrication of organic 
single-crystal FETs is discussed in Chapter 6: electrostatic bonding of a crystal to a prefabricated silicon substrate. In a device fabricated with this technique on a tetracene single crystal, a FET mobility around $1 \mathrm{~cm}^{2} / \mathrm{Vs}$ is measured. This illustrates that the FET performance is comparable to the best existing organic TFTs, due to the fabrication process that does not substantially affect the crystal quality.

The high quality of the FETs is also indicated by the observation of ambipolar transport in copper- and iron-phthalocyanine single crystals. In Chapter 7 we investigate $\mathrm{CuPc}$ and $\mathrm{FePc}$ single-crystal FETs, which exhibit ambipolar operation, despite extrinsic device non-idealities and despite the use of gold as a high work-function metal for the fabrication of source and drain electrodes.

Making use of the compatibility of the electrostatic bonding technique with different types of gate insulators, we next investigate the integration of high$\epsilon$ dielectric materials, like $\mathrm{Ta}_{2} \mathrm{O}_{5}$ or $\mathrm{ZrO}_{2}$, in the organic single-crystal FETs. Chapter 8 expands on a significant technological problem of the application of high- $\epsilon$ dielectrics, i.e. small leakage current through the dielectric, resulting in irreversible degradation of the single-crystal FET performance. A stable FET operation is obtained by an improvement of the dielectric quality, meaning a decrease of the leakage current to values smaller than $10^{-9} \mathrm{~A} / \mathrm{cm}^{2}$.

The combination of the high quality and the high reproducibility of the FET measurements opens new opportunities for investigations. It allows to study how different aspects of the devices influence transistor operation, and additionally, it makes new operation regimes accessible for proceeding research. These topics are represented in the final Chapter 9. A comparative study of rubrene singlecrystal FETs, fabricated using different materials as gate insulator, shows that the mobility of the charge carriers systematically decreases with increasing $\epsilon$

of the dielectric gate insulator: $\mu \propto \epsilon^{-1}$. This finding illustrates that the mobility of carriers in organic single-crystal FETs is an intrinsic property of the crystal/dielectric interface and that it does not only depend on the specific molecule. The second part of the final Chapter discusses experimental FET results at charge densities up to 0.2 holes per molecule, a regime that has so far not been investigated in organic single-crystal FETs. We argue that in this high charge density regime, Coulomb interactions between the charge carriers are significant.

\section{References}

[1] The Power of Invention: Plastics, Newsweek Magazine, 12-1-1998 
[2] Some examples of inorganic polymers are silicones, polysilanes, polygermanes, and polyphosphazenes. Whereas these compounds contain non-carbon elements as the principle backbone, they may have carbon in the side groups.

[3] C. K. Chiang, C. R. Fincher Jr., Y. W. Park, A. J. Heeger, H. Shirakawa, E. J. Louis, S. C. Gau, and A. G. MacDiarmid, Phys. Rev. Lett. 39, 1098 (1977)

[4] In 2000, Alan J. Heeger, Alan G. MacDiarmid and Hideki Shirakawa received the Nobel prize in chemistry for the discovery and development of electrically conductive polymers.

[5] A. J. Heeger, Nobel Lecture, December 8, 2000 (see http://nobelprize.org/chemistry)

[6] J. M. Shaw, and P. F. Seidler, IBM J. Res. \& Dev. 45, 3 (2001)

[7] C. D. Dimitrakopoulos, and P. R. L. Malenfant, Adv. Mater. 14, 99 (2002)

[8] R. C. Haddon, A. S. Perel, R. C. Morris, T. T. M. Palstra, A. F. Hebard, and R. M. Fleming, Appl. Phys. Lett. 67, 121 (1995); J. G. Laquindanum, H. E. Katz, A. J. Lovinger, and A. Dodabalapur, Chem. Mater. 8, 2542 (1996); F. Garnier, A. Yassar, R. Hajlaoui, G. Horowitz, F. Deloffre, B. Servet, S. Ries, and P. Alnot, J. Am. Chem. Soc. 115, 8716 (1993)

[9] E. A. Silinsh, and V. Čápek, Organic Molecular Crystals: Interaction, Localization, and Transport Phenomena (AIP Press, Woodbury, 1994)

[10] M. Pope, and C. E. Swenberg, Electronic Processes in Organic Crystals and Polymers, 2nd ed. (Oxford University Press, New York, 1999)

[11] R. Farchioni, and G. Grosso, Organic electronic Materials (Springer-Verlag, Berlin, 2001)

[12] N. Karl, In: Crystals, Growth, Properties and Applications, Vol. 4, ed. by H. C. Freyhardt (Springer-Verlag, Berlin 1980) pp. 1-100

[13] W. Warta, and N. Karl, Phys. Rev. B 32, 1172 (1985)

[14] N. Karl, K. -H. Kraft, J. Marktanner, M. Münch, F. Schatz, R. Stehle, and H. -M. Uhde, J. Vac. Sci. Technol. A 17, 2318 (1999)

[15] S. M. Sze, Physics of Semiconductor Devices (Wiley, New York, 1981)

[16] A. T. Fiory, A. F. Hebard, R. H. Eick, P. M. Mankiewich, R. E. Howard, and M. L. O’Malley, Phys. Rev. Lett. 65, 3441 (1990); J. Mannhart, J. Bednorz, K. A. Muller, and D. G. Schlom, Z. Phys. B 83, 307 (1991); J. Mannhart, Supercon. Sci. Technol. 9, 49 (1996); N. Chandrasekhar, O. T. Valls, and A. M. Goldman, Phys. Rev. B 54, 10218 (1996); P. Konsin, and B. Sorkin, Phys. Rev. B 58, 5795 (1998); C. H. Ahn, S. Gariglio, P. Paruch, T. Tybell, 
L. Antognazza, and J. -M. Triscone, Science 284, 1152 (1999); C. H. Ahn, K. M. Rabe, and J. -M. Triscone, Science 303, 488 (2004)

[17] S. Mathews, R. Ramesh, T. Vankatesan, and J. Benedetto, Science 276, 238 (1997); H. Ohno, D. Chiba, F. Matsukura, T. Omiyra, E. Abe, T. Dietl, Y. Ohno, and K. Ohtani, Nature 408, 944 (2000); H. Tanaka, J. Zhang, and T. Kawai, Phys. Rev. Lett. 88, 027204 (2002)

[18] D. M. Newns, J. A. Misewich, C. C. Tsuei, A. Gupta, B. A. Scott, and A. Schrott, Appl. Phys. Lett. 73, 780 (1998)

[19] H. -T. Kim, B. G. Chae, D. H. Youn, S. L. Maeng, and K. Y. Kang, New J. Phys. 6, 052 (2004)

[20] H. Ohno, D. Chiba, F. Matsukura, T. Omiyra, E. Abe, T. Dietl, Y. Ohno, and K. Ohtani, Nature 408, 944 (2000)

[21] G. Horowitz, Adv. Mater. 10, 365 (1998)

[22] H. E. Katz, and Z. Bao, J. Phys. Chem. B 104, 671 (2000)

[23] Z. Bao, A. Dodabalapur, and A. J. Lovinger, Appl. Phys. Lett. 69, 4108 (1996); H. Sirringhaus, P. J. Brown, R. H. Friend, M. M. Nielsen, K. Bechgaard, B. M. W. Langeveld-Voss, A. J. H. Spiering, R. A. J. Janssen, E. W. Meijer, P. Herwig, D. M. de Leeuw, Nature 401, 685 (1999)

[24] W. A. Schoonveld, J. Vrijmoeth, and T. M. Klapwijk, Appl. Phys. Lett. 73, 3884 (1998)

[25] G. H. Gelinck, T. C. T. Geuns, and D. M. de Leeuw, Appl. Phys. Lett. 77, $1487(2000)$

[26] G. Horowitz, Adv. Funct. Mater. 13, 53 (2003)

[27] I. H. Campbell, and D. L. Smith, Solid State Phys. 55,1 (2001)

[28] M. C. J. M. Vissenberg, and M. Matters, Phys. Rev. B 57, 12964 (1998)

[29] S. F. Nelson, Y. -Y. Lin, D. J. Gundlach, and T. N. Jackson, Appl. Phys. Lett. 72, 1854 (1998)

[30] V. Podzorov, V. M. Pudalov, and M. E. Gershenson, Appl. Phys. Lett. 82, 1739 (2003)

[31] V. Podzorov, S. E. Sysoev, E. Loginova, V. M. Pudalov, and M. E. Gershenson, Appl. Phys. Lett. 83, 3504 (2003)

[32] R. W. I. de Boer, T. M. Klapwijk, and A. F. Morpurgo, Appl. Phys. Lett. 83, 4345 (2003) 
[33] J. Takeya, C. Goldmann, S. Haas, K. P. Pernstich, B. Ketterer, and B. Batlogg, J. Appl. Phys. 94, 5800 (2003)

[34] V. V. Butko, X. Chi, D. V. Lang, and A. P. Ramirez, Appl. Phys. Lett. 83, $4773(2003)$

[35] V. V. Butko, X. Chi, and A. P. Ramirez, Solid State Commun 128, 431 (2003)

[36] G. Horowitz, F. Garnier, A. Yassar, R. Hajlaoui, and F. Kouki, Adv. Mater. 8, $52(1996)$

[37] M. Ichikawa, H. Yanagi, Y. Shimizu, S. Hotta, N. Suganuma, T. Koyama, and Y. Taniguchi, Adv. Mater. 14, 1272 (2002)

[38] A. F. Hebard, M. J. Rosseinsky, R. C. Haddon, D. W. Murphy, S. H. Glarum, T. T. M. Palstra, A. P. Ramirez, and A. R. Kortan, Nature 350, 600 (1991); O. Gunnarsson, E. Koch, and R. M. Martin, Phys. Rev. B 54, 11026 (1996) 
Chapter 2

\section{Physics of organic semiconductors}

We summarize the theoretical concepts of organic semiconductors needed for a good understanding of the charge transport properties. In addition, we present the standard semiconductor device characteristics, with an emphasis on the interpretation of organic semiconductor experiments. 


\section{$2.1 \quad$ Introduction}

The name "organic" is a historical name, dating back to 19th century, when it was believed that organic compounds could only be synthesized in living organisms through vis vitalis - the "force of life" [1]. In our modern society, though, organic molecules appear in our daily surroundings also in the form of non-living matter, of which the most obvious example is plastic. The commonality in all organic molecules, either natural or synthetic, is that they consist of a configuration of carbon atoms in combination with other atoms, typically hydrogen, nitrogen, and/or oxygen. Within this definition there is an endless variation in molecular structures, and linked to that also a broad range of chemical and physical properties of these materials.

In this Thesis, we mainly focus on the electrical transport properties of organic semiconductor crystals. A comparison with their well-studied inorganic counterparts seems natural. The inorganic semiconductors have covalent or ionic bonds that keep atoms together in the lattice and charge transport is typically described using the single-electron approach. However, organic crystals have a more complicated structural organization, exhibiting two levels. The first level is the intramolecular structure of individual molecules, consisting of covalently bound atoms, and the second level is the intermolecular structure of molecules in the lattice. The complicated structural organization is strongly correlated with the electronic organization, which determines the charge transport properties of organic semiconductors. In particular, the weakly interacting molecular systems are characterized by very strong interactions between the charge carriers and their lattice environment, resulting in polaronic charge carriers: quasi-particles that consist of an electron or hole surrounded by a cloud of electronic polarizations, often also accompanied by molecular and structural deformations $[2,3]$. The polaronic effects "shape" the DC transport properties of organic semiconductors.

In this Chapter we provide the theoretical basis for the analysis of charge transport in Organic Molecular Crystals (OMCs). Following the structural organizational levels in OMCs, we first review the molecules that are used in singlecrystal research (Section 2.2), then we discuss their intermolecular interactions and geometry (Section 2.3). These are related to the charge transport processes in organic semiconductors, which are the focus of Section 2.4, with the main message being the importance of charge carrier interactions with their environment in these weakly bound molecular systems. The final Section 2.5 discusses the principles and theoretical considerations related to charge transport measurements, for which in this Thesis we use a field-effect transistor (FET) geometry. 
<smiles>c1ccc2ccccc2c1</smiles>

naphthalene

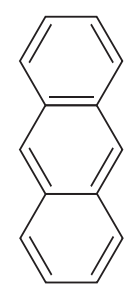

anthracene

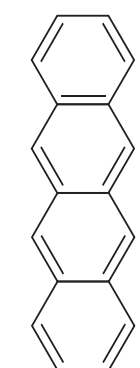

tetracene

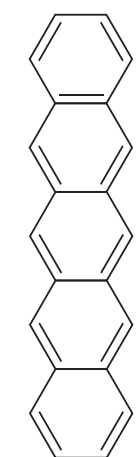

pentacene

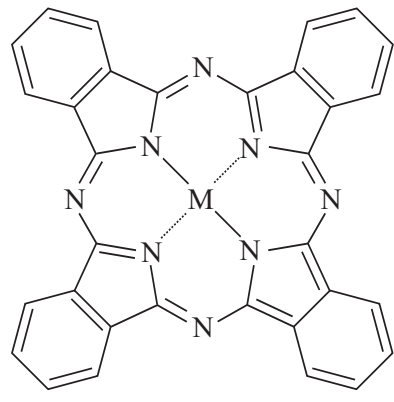

metal-phthalocyanine<smiles>c1ccc(-c2c3ccccc3c(-c3ccccc3)c3c(-c4ccccc4)c4ccccc4c(-c4ccccc4)c23)cc1</smiles>

rubrene

Figure 2.1: Structure of small molecules that are frequently used in investigations on organic semiconductors. The upper row of aromatic molecules (naphthalene, anthracene, tetracene, pentacene) belong to the linear oligo-acenes. Rubrene is a derivative of tetracene, with four H-atoms substituted for phenyl-rings. The heterocyclic metal-phthalocyanine molecule contains, besides $\mathrm{C}$ and $\mathrm{H}$, also nitrogen atoms $(\mathrm{N})$ and a metal element (M) in the center of the molecule.

\subsection{Organic molecules}

Organic single-crystal research mainly focuses on aromatic and heterocyclic molecules, of which Fig. 2.1 shows examples. The aromatic hydrocarbons are planar molecules, consisting of aromatic rings of carbon atoms. Linearly coupled rings form the most studied group, the polyacenes: naphtalene, anthracene, tetracene, pentacene. Derivatives of polyacenes have one or more hydrogen atoms replaced by a side-group, which, in case of rubrene, is a phenyl-group. A selection of well-known heterocyclic molecules are the phthalocyanines, which are planar macrocycles of carbon and nitrogen atoms. In metal-phthalocyanine the organic macrocycle serves as a ligand and has a metal atom in the center. Of the many metal derivatives that are known $[4,5]$, in this Thesis, we will investigate copperphthalocyanine and iron-phthalocyanine. 


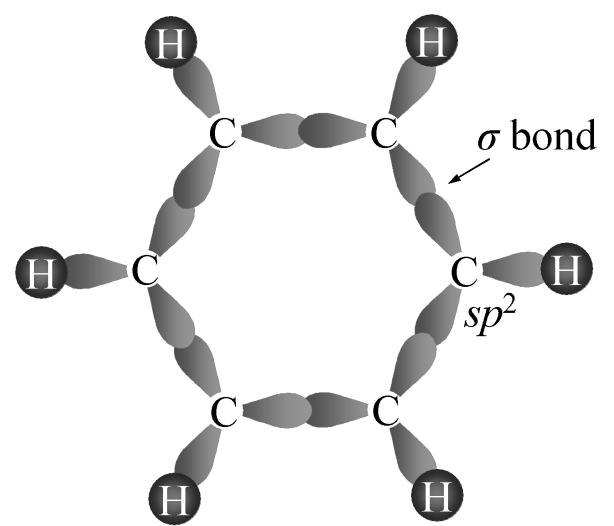

(a)

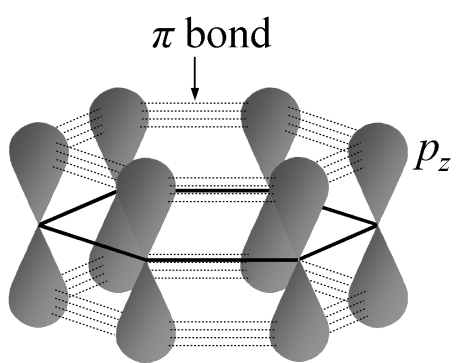

(b)

Figure 2.2: Illustration of the $\sigma$ and $\pi$ bonding in the aromatic benzene molecule. (a) Top view: overlapping $s p$ orbitals form $\sigma$ bonds in the plane of the molecule (for clarity carbon atoms $(\mathrm{C})$ and hydrogen atoms $(\mathrm{H})$ are indicated). (b) Side view: sideways overlapping $p_{z}$ orbitals constitute an extended $\pi$ system with a high electron density above and below the molecular plane.

The aromatic and heterocyclic molecules have large similarities in their electronic structure. In order to discuss these, let us first zoom in on the electronic organization of the building blocks of organic molecules: the carbon atoms. The electrons in each carbon atom are divided over states that are characterized by a wavefunction, described as an orbital, and a discrete energy. The core electrons in the $1 s$ orbital, strongly bound to the atom, are insignificant for the atomic and molecular interactions and, therefore, we further ignore them. The outermost carbon electrons, situated in the $2 s$ and the $2 p$ orbital, are only loosely bound to the atom. In aromatic and heterocyclic molecules, a mixing of these orbitals takes place, called an $s p^{2}$ hybridization, and results in three equivalent $s p$ orbitals and one $p_{z}$ orbital.

The covalent bonds between the atoms in their molecular structure are formed by overlapping $s p$ orbitals, resulting in $\sigma$ bonds, and overlapping $p_{z}$ orbitals, forming $\pi$ bonds (Fig. 2.2). The molecular structure of aromatic and heterocyclic molecules are characterized by an alternation of single $(\sigma)$ bonds and double ( $\sigma$ plus $\pi$ ) bonds, as visible in Fig. 2.1. Notice that Fig. 2.1 shows only one possible drawing of each molecule: interchanging the positions of the single and double bonds does not fundamentally change the molecule but merely represents a different resonance structure (see Fig. 2.3a). In reality, the molecular structure is a linear combination of all possible resonance structures, and as a consequence the $\pi$ bonds are spread out over the molecule. Molecules with such a system of delocalized $\pi$ bonds are called conjugated. 


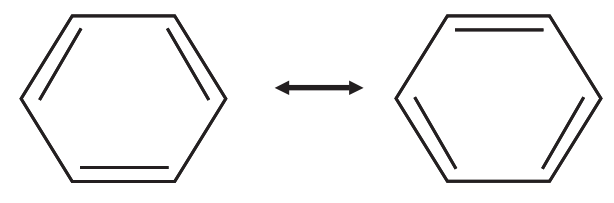

(a)

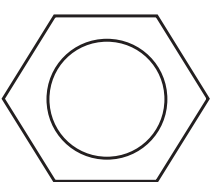

(b)

Figure 2.3: (a) Similar to other conjugated molecules, the molecular structure of a benzene molecule is often represented as a hexagonal ring of alternate single and double carbon-carbon bonds. This figure shows the two molecular resonance structures, or Kekulé structures, with interchanged positions of single and double bonds, indicating the delocalization of the $\pi$ bonds. (b) An alternative representation of the benzene molecule that indicates the delocalization of the $\pi$ bonds, by placing a circle inside the hexagonal ring.

The $\pi$ electrons that are involved the $\pi$ bonds of conjugated systems are responsible for many chemical and physical properties of the molecules. The lowest energy reactions and excitations are determined by the Highest Occupied Molecular Orbital (HOMO) and the Lowest Unoccupied Molecular Orbital (LUMO). In aromatic and many heterocyclic molecules the HOMO and LUMO have $\pi$ character, whereas the stronger bonding $\sigma$ bonds are lying deeper in energy. Therefore, knowledge of the $\pi$ system is important for understanding properties of the molecule and also, as we will see later, of the crystal.

Interesting characteristics can be derived from the shape of the $\pi$ electronic wavefunction. In the planar aromatic and heterocyclic molecules, the atomic $p_{z}$ wavefunctions have a density profile that is shaped as two lobes oriented perpendicular to the $\sigma$ bonds, thus out of the molecular plane. The sideways overlap of the $p_{z}$ orbitals results in a $\pi$ system that is most dense above and below the plane of the molecule, with nodes in the molecular plane. Moreover, the extensive sideways overlap of each $p_{z}$ orbital with all neighboring $p_{z}$ orbitals produces a system of $\pi$ electrons that are delocalized over the whole molecule. In short, the conjugated molecules possess clouds of delocalized $\pi$ electrons situated above and below the molecular plane, as visible in Fig. 2.2b.

The extension of the $\pi$ system over the molecule improves the conducting properties of the individual molecule. Notice that the larger the molecule, the more delocalized the $\pi$ electrons are. For this reason, the fabrication of polymeric Thin-Film Transistors (TFTs) focuses on the use of conjugated polymers $[6,7,8]$. Unfortunately, conduction in these polymer TFTs is limited by disorder and by the inter-chain hopping of charge carriers from one polymer chain to the neighboring chain. It illustrates that besides the extension of the $\pi$ system, also 
other aspects affect the conductivity, such as the intermolecular organization.

Interestingly, the delocalization of the conducting $\pi$ electrons is unaffected by substitution of specific side-groups. A good example are the substituted phenylgroups in rubrene, which do not electronically interact with the $\pi$ system of the tetracene backbone. Such a substitution can, without affecting the molecular conductivity, result in a changes in the OMC crystal structure and thus influence the crystal conductivity (see Section 2.3.2). However, not all side-groups leave the $\pi$ system intact. The conjugation of the molecule can be distorted by substitution of double bonded side-groups, like the O-atoms in pentacenequinone [9]. Also, single bonded highly electronegative side-groups attract $\pi$ electrons and localize them on the side-group.

Despite the fact that, in this Chapter, we focus mainly on the $\pi$ electronic system and its relation to the crystalline charge transport properties, it is worth noting that in the metal-phthalocyanines, additional to the $\pi$ system, also the metal atomic orbitals play an important role [5]. Depending on the type of metal, the metal valence orbitals are often lying close in energy to the highest occupied and lowest unoccupied $\pi$ orbitals of the organic ligand. The metal orbitals and the ligand orbitals partly hybridize, but typically they retain most of their original character. Thus the HOMO and LUMO are generally characterized as mostly metallic or mostly $\pi$, i.e. localized on the metal atom or extended over the ligand.

\subsection{Intermolecular organization}

We look separately at two aspects of the intermolecular organization: the intermolecular interactions and the crystal structure. Note that these two items are strongly intertwined. The strength of the force between molecules in a crystal lattice is expressed in the interaction energy. The orientation of the molecules in the lattice is such that the summed interaction energy of all molecular interactions in the lattice is optimized. Thus, the intermolecular interactions play an important role in the formation of the crystal structure.

\subsubsection{Intermolecular interactions}

The attractive force that binds molecules in a lattice is weak, much weaker than the intramolecular covalent bonds, and therefore, molecular properties are hardly affected by the intermolecular interactions. Also, the forces holding molecules together in a crystal are much weaker than the covalent and ionic bonds found in inorganic crystals. This manifests itself in the mechanical and elastic properties of molecular crystal, e.g. low melting and sublimation temperatures, a large thermal 


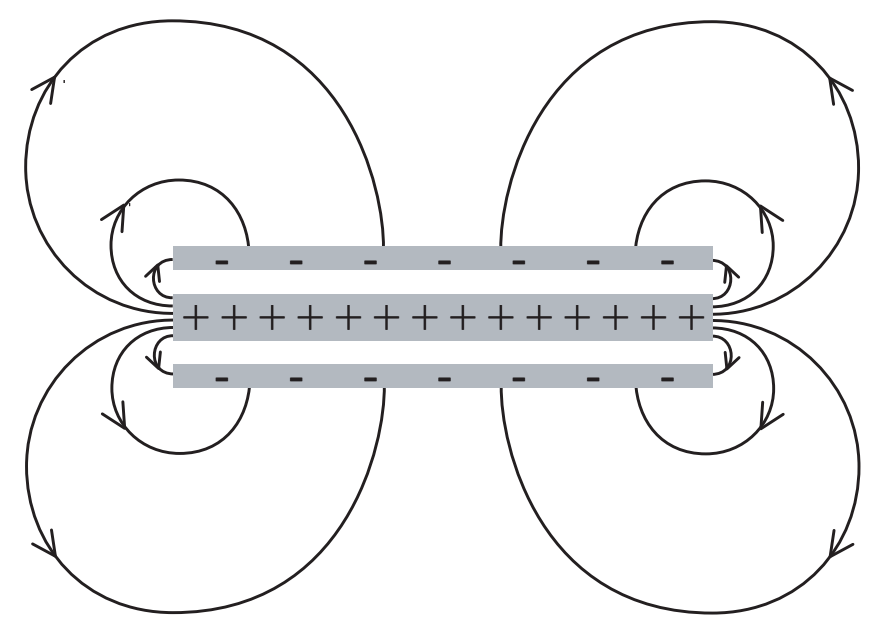

Figure 2.4: Schematic picture of a conjugated molecule and its quadrupole field. The plane of the molecule is indicated as an area (grey square) with a net positive charge, and the $\pi$ clouds above and below the plane are areas with a net negative charge. The quadrupolar field lines, shown as black lines with arrows, are most dense at the periphery of the molecule.

expansion and low mechanical strength (i.e. organic crystals are typically very brittle).

Intermolecular interactions in molecular crystals are often referred to as van der Waals interactions $[2,3]$. The van der Waals force is a nonvalent interaction between the electrically neutral molecules. The main contribution to this attractive force comes from the interaction of a fluctuating dipole moment on a molecule, induced by the electron motion within the molecule, with the induced dipole moment on a neighboring molecule. The strength of the dipole/induceddipole interaction depends on, among other things, the molecular polarizability. In aromatic and heterocyclic molecular crystals, the weakly bound and thus easily polarizable $\pi$ electrons provide relatively strong van der Waals interactions.

In aromatic and heterocyclic molecules, additional to the van der Waals interactions, the intermolecular interaction strength holds a significant contribution provided by static quadrupole-quadrupole interactions $[10,11]$. They arise from the permanent quadrupole moments that exist on molecules with an extended $\pi$ system. The net negative charge in the $\pi$ clouds above and below the plane of the molecule, combined with the net positive charge in the plane of the molecule, resembles two dipoles oriented head to head. This results in a quadrupolar field around the molecule, as schematically drawn in Fig. 2.4. Interactions via these static fields provide a considerable share of the total intermolecular interaction strength. Also higher order multipole moments are induced by the molecular 
orbitals, but they realize a less significant contribution to the intermolecular interaction strength.

The van der Waals force and the quadrupolar interaction promote two competing orientations of the molecules in the crystal lattice. The van der Waals interaction favors face-to-face orientation of the planar molecules in the lattice, as this maximizes the van der Waals interaction energy. The force between the static quadrupole fields, however, is strongest for an edge-to-face orientation of the molecules. The interplay between the two types of interactions is typically expressed in the crystal structure of conjugated systems [10, 11]. For example, in tetracene and pentacene, it results in a herringbone-arrangement of the molecular layers, which we will discuss in the next Section.

The intermolecular interactions play a dominant role for the charge transport properties. The strength of the forces between molecules determines how close the molecules will approach each other and this influences the extent of $\pi$ orbital overlap between neighboring molecules. Moreover, the $\pi$ overlap is affected by the orientation of the molecules $[12,13]$, which is a result of the ensemble of van der Waals and quadrupole interactions. The $\pi$ orbital mixing causes broadening of each discrete molecular energy level into an energy band. In general, the HOMO (resp. LUMO) of the molecule transforms into the valence band (resp. conduction band) of the molecular crystal. Thus, the intermolecular interactions affect the bandwidth of the valence and conduction band, i.e. the bands that are responsible for the crystal conductive properties.

\subsubsection{Crystal structure}

Despite the knowledge that large conjugated molecules have a more extended $\pi$ system, single-crystal research mainly focuses on small molecules. The reason is technological: in general, the smaller and the more symmetric the molecules are, the easier they arrange into a crystalline lattice.

But even the relatively small and symmetric molecules, like tetracene, have a crystal structure that is rather complicated and of low symmetry. It is simply expressed by the triclinic crystallographic structure of tetracene, i.e. the unit cell has three unequal dimensions $(a \neq b \neq c)$ and three unequal angles $(\alpha \neq \beta \neq$ $\gamma \neq 90^{\circ}$ ). Fig. 2.5 shows the crystal structure of tetracene, clearly visualizing a layered organization. That is, the molecules are organized in two-dimensional planes, each one molecule thick, which are separated by narrow planes of 'empty' space. Tetracene molecules are standing almost upright within each monolayer, making an angle of only $\sim 10^{\circ}$ with the normal. From top view, the tetracene molecules are oriented in a herringbone-structure. 


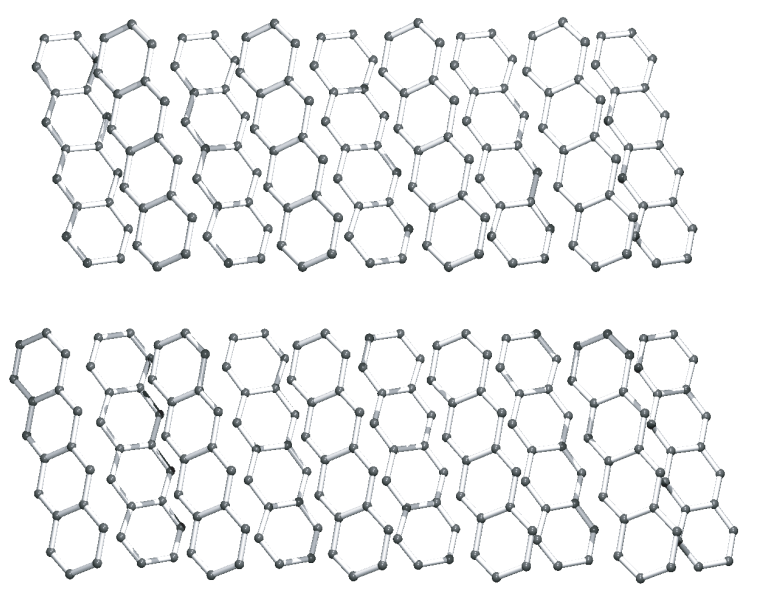

(a)

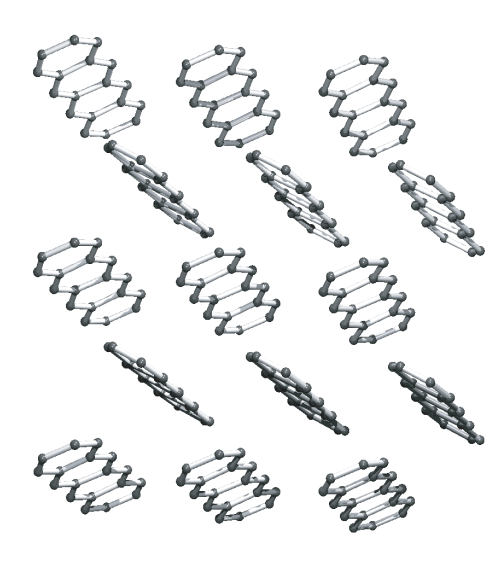

(b)

Figure 2.5: The crystal structure of tetracene: (a) viewed along the $a$-axis, which visualizes the layered organization, and (b) a top view of the $a b$-plane, showing the herringbone configuration.

The low symmetry of the crystal lattice contributes to an anisotropy of the crystal properties, like electrical conductivity [13]. As a rule of thumb, the distance between, and the orientation of, the molecules give a good indication of the strength of the $\pi$ orbital overlap. Accordingly, since the overlap of the molecular $\pi$ orbitals is larger within a molecular plane (the $a b$-plane) than perpendicular to that, in between two planes (the $c^{*}$-direction), the conductivity in the $a b$-plane is expected to be higher than in the $c^{*}$-direction. The conductivity anisotropy within the $a b$-plane is anticipated to be less pronounced. This is a simplistic picture, though, and for a quantitative determination of the overlap integral one should also include the relative phase of the wavefunctions of involved orbitals [14]. Anisotropy of charge carrier mobilities has in fact been experimentally observed in ultra-pure single crystals of, e.g., naphthalene and anthracene, in time-of-flight experiments by Karl et al. [15]. Additionally, in experiments on rubrene field-effect transistors, an anisotropy of the conductivity in the rubrene crystalline $a b$-plane has been observed, and is also attributed to the directiondependent orbital overlap [16].

A comparison of tetracene with rubrene (see Fig. 2.6) illustrates that the crystal structure can change remarkably as a result of the substitution of sidegroups. Also rubrene has a triclinic crystal structure, built up of molecular layers with a herringbone-pattern of molecules in each layer. However, the orientation of the molecules in the layer differs considerably from the case of tetracene, due 
to steric hindrance of the phenyl side-lobes [17]. Instead of standing upright, the tetracene-backbone in rubrene lies in the molecular plane. The phenyl side-groups are sticking out above and below the tetracene-backbone.

The different crystal structure of rubrene is expected to cause different conducting qualities of the rubrene OMC $[18,19]$. Especially in the crystalline $b$ direction, the orbital overlap of rubrene molecules is considerably larger than for tetracene: almost $50 \%$ of the $\pi$ clouds of nearest neighboring rubrene molecules are directly facing each other. The stronger orbital overlap is believed to account for the ( $\sim 20$ times) larger mobility that is measured in rubrene single-crystal FETs [20] than in tetracene single-crystal FETs [21, 22].

The herringbone crystal structure of metal-phthalocyanines, also shown in Fig. 2.6, is characterized by columnar stacks of the disc-like molecules, aligned along the crystalline $b$-direction. The molecules possess a remarkably large $\pi$ electronic system, containing 42 electrons, which overlaps strongly in the direction parallel to the stacks. Thus, the conductivity is expected to be especially large along the $b$-direction, and a strong anisotropy is foreseen.

Because of a weak interaction between the molecules, polymorphism is a common phenomenon in organic materials: the molecular packing and the shape of organic crystals can be easily affected by the growth conditions. For example, the thiophenes exhibit two different structures depending on the growth temperature [23]. For pentacene, no less than four polymorphs have been reported by Mattheus et al. [24], all of which are layered herringbone structures. In thin films, the type of pentacene polymorph(s) that is (are) grown depends on parameters like the substrate material, the substrate temperature and the thickness of the grown film. In pentacene single crystals that are grown by the PVT method (see Chapter 3), they find only one polymorph. The effect of polymorphism on the crystalline charge transport properties is reported in Ref. [12], where the difference in polymorphic pentacene structures are associated to disparities in the calculated bandwidths.

In many cases, organic molecular crystals exhibit one or more structural phase transitions upon lowering the temperature. For the study of single-crystal fieldeffect transistors at low temperature, the occurrence of a structural phase transition can be detrimental. In tetracene crystals, for instance, a structural phase transition occurs below $200 \mathrm{~K}$ (see, e.g., [25]). Co-existence of two crystallographic phases at lower temperatures causes the formation of grain boundaries and stress, which are responsible for the trapping of charge carriers. In tetracene, in addition, occurrence of the structural phase transition often results in cracking of the crystals with cooling and a consequent device failure. 


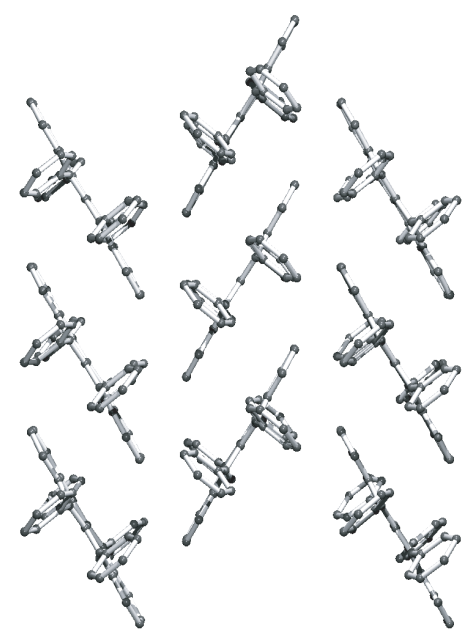

(a)

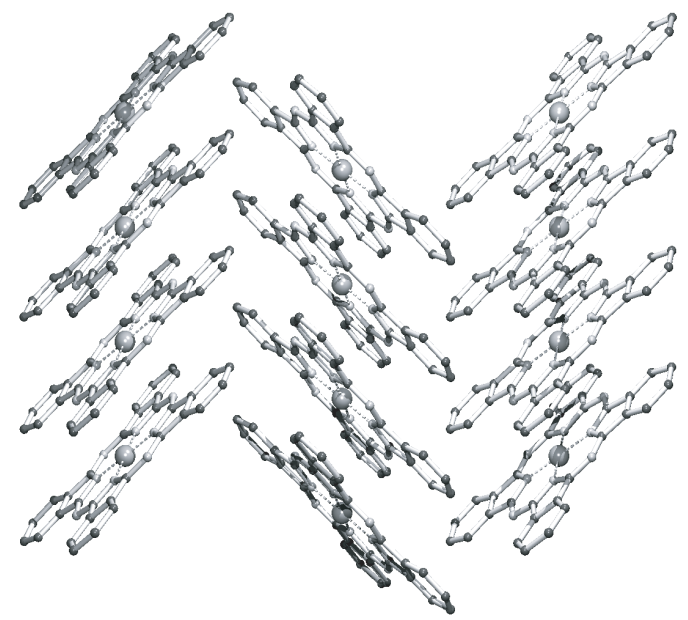

(b)

Figure 2.6: A top view of the $a b$-plane crystal structure of (a) rubrene and (b) copperphthalocyanine. The herringbone configuration is apparent in both.

\subsection{Charge transport mechanism in $\pi$ systems}

Crystals of small conjugated molecules belong to the class of organic semiconductors. Each molecular $\pi$ system in the crystal interacts with $\pi$ systems on neighboring molecules, and the mixing of these $\pi$ systems results in the formation of energy bands in the crystal. The crystal valence band originates from overlapping HOMO's of the individual molecules, whereas the conduction band is a mixing of LUMO's. Organic molecular HOMO levels are generally fully occupied, resulting in a completely filled valence band and an empty conduction band, which explains the semiconducting properties of the OMCs.

The organic semiconductors are characterized by wide bandgaps and small bandwidths. For most pure single-component crystals, the semiconducting energy gap is in the range of $1-4 \mathrm{eV}[3,15]$, approximately equal to the HOMO-LUMO gap of the individual molecules. Due to this wide energy gap, only a low density of electrons is thermally excited from the valence band to the conduction band. The density of mobile charge can be significantly enhanced by chemical or electrostatic doping, the latter of which is applied in a field-effect transistor geometry, as applied in this Thesis. The molecular crystal's bandwidth is related to the orbital overlap strength of the HOMO's and LUMO's. Typically, calculations show that the conduction and valence bands for pure and defect-free OMCs are narrow, $0.1-0.5 \mathrm{eV}[3,26,27]$.

The narrow bandwidths have important consequences for the charge trans- 
port in organic semiconductors, as they result in low charge carrier mobilities and strong interactions between the charge carriers and their local crystal environment. These two consequences are intertwined. The strong interaction between a carrier and its environment is induced by the low speed, resulting from the low mobility, of the carrier. But also, the mobility is further reduced by the localization of charge carriers that results from their interaction with the surrounding crystal. The following Subsections discuss these two aspects of charge transport in more detail - the charge carrier mobility and the carrier interaction with its environment.

Note that during the discussion in this Section we make a distinction between intrinsic and extrinsic aspects of charge transport. The subjects discussed in the fist two Subsections are of intrinsic origin, i.e. they do not stem from the presence of disorder and they play a role in the ultimate electrical performance limits of OMCs. Naturally, these are the aspects that we are interested in when studying fundamental charge transport processes in OMCs. However, the intrinsic properties of OMCs can be disguised by extrinsic effects: the presence of defects. The limiting effect of defects on the OMC transport properties are discussed at the end of this Section.

\subsubsection{Intrinsic mobility}

For the charge transport studies on organic single crystals in this Thesis, we measure the electrical conductivity of the crystals under varying circumstances. The conductivity in a (molecular) solid depends on two parameters, i.e. the charge carrier density $n$ and the charge carrier mobility $\mu$ :

$$
\sigma=n e \mu
$$

We control the charge carrier density by using the field-effect transistor geometry - the gate-field induced charge can be changed by simply turning a knob. The mobility, though, cannot be controlled: it is a material parameter. It is defined as the magnitude of the drift velocity of a charge carrier per unit electric field, $\mu=v_{d} / E$. In other words, the mobility is a measure of the ease with which the charge carriers migrate through the solid.

The charge carrier mobility in OMCs is generally low compared to most inorganic semiconductors. The mobility is strongly related to the width of the energy band in which the charge carrier is situated, which is small in organic semiconductors. For comparison: band structure calculations on OMCs have generated bandwidths of $0.1-0.5 \mathrm{eV}[3,26,27]$, whereas the bandwidth in silicon is $\sim 10$ 
$\mathrm{eV}$. Consequently, the mobility in crystalline $\mathrm{Si}$ is more than two orders of magnitude higher than the mobility in organic single crystals, which is of the order of $1-10 \mathrm{~cm}^{2} / \mathrm{Vs}[20,28,29,30,31]$.

The value of the mobility, and its temperature dependence, give an indication of the type of charge transport in a material: band-type or incoherent hopping. Coherent band-type transport is found in conductors of which the charge carriers can be described as delocalized wave packets, where typically the wavevector $k$ is a well-defined quantum number. The curvature of the energy band in $k$-space gives the charge carrier's effective mass $m^{*}$. The mobility is generally high, e.g. $\sim 500-5000 \mathrm{~cm}^{2} / \mathrm{Vs}$ for crystalline $\mathrm{Si}$, and it can be expressed in terms of $\mathrm{m}^{*}$ and the scattering time $\tau$ of the charge carriers:

$$
\mu=\frac{e}{m^{*}} \tau
$$

At increasing temperature, the scattering time $\tau$ decreases due to the thermal excitation of lattice vibrations (phonons), which act as scattering sites. As a result, the mobility has a power-law T-dependence:

$$
\mu \propto T^{-n}, n \geq 1.5
$$

In the case of localized charge carriers, transport is incoherent: an uncorrelated sequence of waiting times and jumps, so that the concept of quasi-continuous acceleration breaks down and Eq. 2.2 and 2.3 do not apply. The charge carriers are not very mobile, because energy needs to be applied to make a charge carrier hop from one site in the lattice to the next. This energy is provided by phonons, which obviously play a role here that is very different from the case of a band-type conductor. The T-dependence of the mobility follows an Arrhenius relation:

$$
\mu \propto \mu_{0} \exp \left(-\frac{E_{a c t}}{k_{B} T}\right)
$$

Both the power-law dependence and the thermally activated dependence of the mobility on temperature have been observed in experiments on organic semiconductors. A clear power-law dependence was found by Karl and coworkers in Time-of-Flight (TOF) experiments on several ultra-pure organic single crystals $[15,28]$, of which an example is shown in Fig. 1.2. An exponential decrease of the mobility of field-induced charge carriers with lowering temperature is typically reported for organic TFTs $[32,33]$. Moreover, several times, a behavior that is in between these two cases has also been observed, that is, a temperatureindependent mobility or a non-monotonous mobility as a function of temperature $[20,21,33,34,35]$. An experimentally determined rule of thumb is that signatures of non-activated transport are found in crystals with a room temperature 
mobility of the order of $\sim 1 \mathrm{~cm}^{2} / \mathrm{Vs}$ or higher, whereas devices with a lower mobility generally exhibit a purely thermally activated behavior.

The high-quality TOF data of Karl et al. [28] have played, and still play, a prominent role in the development of a microscopic description of the charge transport in organic materials $[2,15]$. The data seem to suggest that coherent transport characterizes the intrinsic charge transport in ultra-pure organic single crystals. However, the applicability of the coherent transport model is at odds with calculations of the charge carrier's mean free path $l$ from experimentally obtained mobilities, which generally is equal to or smaller than molecular distance $a[2,3]$ :

$$
l \leq a
$$

This means that an important criterion for the validity of the delocalized singleelectron model is not fullfilled. These seemingly contradicting observations indicate that charge transport in OMCs is in a grey area between obvious band-type behavior and purely incoherent hopping transport. Also other observations point out this conclusion. In several of the TOF measurements, the power-law dependence disappears at increased temperature (e.g. at $T>100 \mathrm{~K}$ for electrons in the $c^{\prime}$-direction of naphthalene [15]). Moreover, the fact that the power-law temperature dependence is not seen regularly in experiments indicates how close the charge carriers are to becoming predominantly localized, either by intrinsic or by extrinsic effects [36].

The conclusion that OMCs reside in a regime where band-type and incoherent hopping transport coexist is generally considered to be a manifestation of the strong interactions that are present in OMCs between charge carriers and their crystal environment. In the narrow-band molecular solids, polarization effects favor localization of charge carriers and narrow the bandwidth even further. The resulting self-trapped electronic states, the polarons, differ in their behavior from (nearly-)free electrons (causing band-type behavior) and they differ as well from strongly localized electrons (inducing incoherent hopping type of behavior). The polaron formation and transport is further discussed in the following Section.

\subsubsection{Polaron transport}

In the narrow-band molecular solids, the charge carriers bear a strong coupling to their crystal environment. Solids with a strong charge-lattice coupling are characterized by polaronic charge carriers, that is, the electrons and holes are dressed with a polarization cloud that follows the charge as it moves through the crystal. This Section treats the polaron formation in OMCs and discusses 
the intricacy of developing a well-defined microscopic description for polaron transport in OMCs.

A polaron is a charge carrying entity consisting of a charge plus the distortion it induces in the local crystal environment. Due to the complicated electronic and structural organization in OMCs, the polarization cloud around a charge concerns several types of processes: polarizations of the electronic structure, molecular distortions, and lattice deformations. The first is a polarization of the electronic orbitals on molecules surrounding the excess charge, which is mainly created by the interaction of the charge with induced dipole moments and with permanent quadrupole moments on surrounding molecules [2]. The molecular distortions are vibrations of the intramolecular covalent bonds, whereas lattice deformations are phonons, i.e. translational and librational motions of the molecules in the crystal lattice.

Following the argumentation by Silinsh and Čápek in Ref. [2], an indication for the strong coupling of carriers to the induced polarizations in their OMC environment comes from a simple comparison of energy scales. Each polarization process is associated with a characteristic time for relaxation of the environment to the distorted state, and a comparison with the charge carrier's mean hopping time $\tau_{h}$ from one molecule to its neighbor indicates whether the carrier resides long enough to induce the involved polarization. The comparison of relaxation time scales can be converted to a comparison of characteristic energy scales using the quantum mechanical uncertainty principle. For instance, the hopping time $\tau_{h}$ is related to the nearest neighbor transfer integral $J$ as:

$$
\tau_{h} \simeq \frac{\hbar}{J}
$$

and similarly, the relevant relaxation times for the different polarization processes are related to their characteristic energy scale. The characteristic energy for the electronic polarization of the orbitals of neighboring molecules corresponds, in first approximation, to the excitation energy $E_{\text {ex }}$ of an electronic exciton [2], and has a value in the order of the HOMO-LUMO gap, $1-4 \mathrm{eV}[3,15]$. Since $J \simeq 0.1-0.5 \mathrm{eV}[3,26,27]$, i.e. in the order of the bandwidth, a comparison leads to: $J \ll E_{\text {ex }}$, thus $\tau_{h} \gg \tau_{e}$ (with $\tau_{e}$ the relaxation time for electronic polarization), indicating a strong coupling of the charge carriers to the electronic polarizations. Similarly, drawing a parallel between $J \simeq 0.1-0.5 \mathrm{eV}$ and the characteristic energies of molecular vibrations, $E_{v} \simeq 0.05-0.3 \mathrm{eV}[2,3]$, and lattice distortions, $E_{l} \simeq 0.03-0.1 \mathrm{eV}[2,3]$, shows that they are very close in energy, which anticipates an additional coupling to molecular and lattice polarizations. The coupling between charge carriers and their environment in OMCs is much 
stronger than in silicon, which is due to the considerably larger Si bandwidth of $\sim 10 \mathrm{eV}$.

Besides the comparison of energy scales, indications for polaron-formation in organic molecular crystals also stem from photo-emission experiments [3]. Photoemission data from single crystals generally show lines that are broadened and shifted in energy relative to the corresponding gas phase spectral lines. The broadened solid-state photoemission lines are suggested to originate from fluctuations in the intermolecular electronic polarization. The spectral line shift is explained by the increased binding energy of a charge in the molecular crystal with respect to a charge on a gas-phase molecule, due to polarizations in the local crystal environment.

The microscopic description of polarons is complex. The polaron is in fact a self-trapped electronic state, as the charge carrier minimizes its energy by creating the local distortions. The charge and its induced polarization cloud form a many-body interaction phenomenon and therefore, single-electron approximation is not valid and inapplicable for the description of electronic states in OMCs. An alternative description requires the involvement of the many different interaction processes that play a role in the polaron formation.

Numerous investigations have been published on the description of polaron transport in OMCs. In many reports, the problem is attacked by solving a (lowelectron-density) Hamiltonian of the form:

$$
H=H_{\mathrm{e}}+H_{\mathrm{pol}}+H_{\mathrm{e}-\mathrm{pol}}
$$

where $H_{\mathrm{e}}$ describes the energy of the charge carriers in the unpolarized OMC, $H_{\text {pol }}$ represents the energy of the induced polarizations in the local environment and $H_{\mathrm{e}-\mathrm{pol}}$ describes the interaction energy between the charge carriers and their surrounding polarizations. Besides the different methods that can be used to solve this Hamiltonian [37, 38, 39], the main differences between the various reported research works concern the simplifications that are made in the second and third term of the Hamiltonian, which affect the transport behavior that is predicted by the model [2].

For simplicity, most authors choose to focus their calculations on only one of the three polarization processes, i.e. they study the electronic polaron (or Coulomb polaron) [40, 41], or the molecular polaron [42], or the lattice polaron $[39,43,44,45,46]$. But even studies that concern the same type of polarization can differ considerably, due to the various ways in which a charge and the induced polarization can interact. In terms of the tight-binding model, the effect of the polarization can be included either by coupling to the on-site energies, or by coupling to the off-diagonal elements, which correspond to the charge transfer 
integrals. A well-known study of the diagonal charge-phonon coupling is the work of Holstein [42], whereas the off-diagonal interaction is investigated for example in the work of Su, Schrieffer and Heeger [47]. Additionally, the published polaron models differ in the radius of action that is chosen, which specifies whether only on-site interactions are included, or also long-range interactions. Finally, other factors, like the anisotropy of the organic crystal lattice, complicate the polaron description even further $[2,48]$.

Despite the extended interest, the literature still lacks a unifying picture providing a clear physical description of the phenomena of polaron formation and polaron transport. This is mainly due to the very complicated character of the many-particle interactions involved in polaron formation. An additional cause is that hardly any experimental information is available on intrinsic electronic transport properties of organic semiconductors, beyond the high-quality data of Karl et al. [15, 28, 49] from low-charge-density TOF measurements on bulk organic crystals.

\subsubsection{Localization by defects}

So far in this Chapter, we have discussed intrinsic properties of OMCs. The intrinsic properties can be disguised by the influence of defects in the crystal, i.e. chemical impurities or structural defects. Every defect acts as a scattering site for charge carriers, because it causes a distortion in the, ideally, periodic lattice potential. Coherent band-type charge transport is impeded by the additional scattering processes, because it decreases the scattering time $\tau$, which is expressed in a reduced conductivity (Eq. 2.2). In an OMC, though, the mean free path of a charge carrier is of the order of the lattice constant, and an increase of the amount of scattering sites due to the presence of defects (for instance 1 defect in 100 sites) is then negligible. The effect of a defect on charge transport is much stronger if it additionally acts as a trapping site.

Trapping is relevant if the defect induces one, or more, energy levels in the bandgap of the organic crystal. A passing charge carrier prefers to occupy this lower energy state, and the trap localizes the charge carrier at its site. Considering the activation energy that is needed to free the charge carrier, traps can be split in shallow traps, when the activation energy is in the order of $k_{B} T$, and deep traps, when the activation energy is outside the range of thermal excitation. Deep traps are often caused by chemical impurities incorporated in the crystal, such as oxidized molecules or molecules that are side products of the main compound's synthesis process [15].

The common concept for deep and shallow traps is that they limit charge 
transport by localization of charge carriers $[2,3]$. This causes a lower density of mobile charges, since trapped carriers are (temporarily) localized at the defect site, and additionally, it can also affect the mobility of the charge carriers at the Fermi level. Depending on the amount of defects, and how strongly they localize the charge carriers, their influence can completely dominate the transport properties. In that case, the experimentally obtained mobility does not equal the intrinsic mobility: it is limited by the presence of defects, and therefore, it is lower than its intrinsic value. Also the temperature dependence of the (extrinsic) mobility expresses the localized nature of the charge carriers: charge transport is thermally activated and described by Eq. 2.4.

The thermally activated temperature dependence is usually found in ThinFilm Transistors (TFTs) on polymers and small molecules. For polymers this is easy to understand: often referred to as a "spaghetti" of long molecules, there is little or no periodic structure in a polymer thin film. With so many trapping and scattering sites, polymers contain bands of localized states. Contrary to polymers, it is possible to crystallize small molecules, which generally results in higher mobility values for the small molecule TFTs. But still, besides a sporadic exception (see Fig. 1.3 and Ref. [33]), the general temperature dependent behavior is thermally activated [32]. It indicates that charge transport is dominated by defects, which in case of small molecule TFTs are often grain boundaries and defects at the organic/dielectric interface due to the lattice-mismatch.

The influence of defects can be neutralized by increasing the charge carrier density. When a trap is filled with a charge carrier, it cannot trap another carrier and has thus become ineffective. Consequently, the higher the charge density, the more low-energy localized states are filled and the more mobile the charge carriers at the Fermi level are. This phenomenon is generally observed as a gatefield dependent mobility in TFTs [32]. In organic single-crystal FETs however, the mobility is often found to be independent of the gate, typically above a certain gate voltage (see Chapter 6). This is considered as an indication of a low defect density, and therefore a high quality, of the organic FET.

\subsection{Principles of charge transport experiments}

\subsubsection{Charge injection into organic semiconductors}

Current injection from metallic contacts into organic solids is a subject of considerable technological importance. Metal/organic interfaces are very important for organic devices, because they can influence the type and the amount of charge injected into the undoped wide-bandgap organic layer. Moreover, for the inves- 


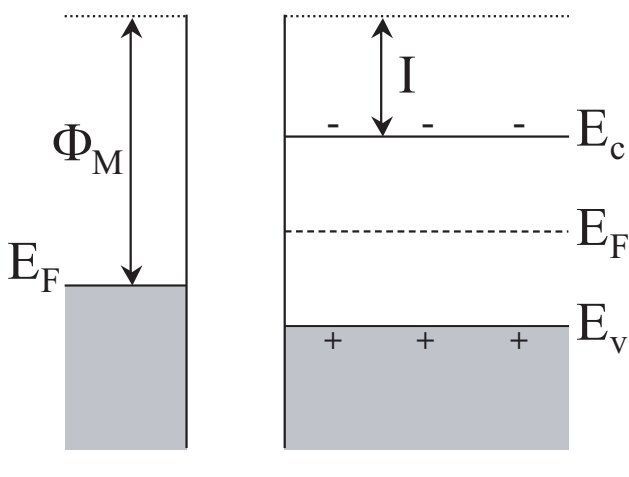

(a)

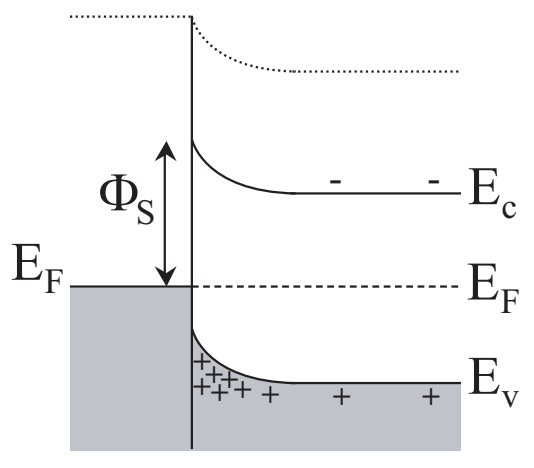

(b)

Figure 2.7: Energy-band diagrams under thermal equilibrium for (a) a metal (left) and an intrinsic semiconductor (right) that are not in contact; (b) a metal/semiconductor contact, with a band bending region in the semiconductor, close to the interface with the metal. $E_{c}$ and $E_{v}$ indicate the edge of the conduction band and the edge of the valence band; $E_{F}$ indicates the position of the Fermi level.

tigation of intrinsic transport properties it is crucially important that measured currents are transport limited, and not injection limited. In this Section we will only touch upon a few of the most frequently used considerations about the interface area.

The ideal contact is an Ohmic contact: a non-limiting source of charge carriers that does not affect the device characteristics. The Ohmic contact itself has a linear and symmetrical $I-V$ relation. In experiments, the metal/organic interface is often found to be non-Ohmic, and despite many investigations, it is not precisely known yet how to microscopically describe the metal/organic interface. Therefore, concepts that are established for the interface of metals with inorganic semiconductors, like Schottky barriers, are generally used as a starting point for modelling.

One of the fundamental aspects of the metal/inorganic semiconductor interface is Fermi level alignment, described by the Mott-Schottky model [50]. When a neutral metal and a neutral semiconducting solid are brought in contact, the Mott-Schottky model predicts that their bulk Fermi levels will align, causing band bending in the semiconducting solid (as visualized in Fig. 2.7). Due to the band bending at the metal/semiconductor interface, a non-Ohmic Schottky barrier can be formed for electrons (holes) when the Fermi level in the semiconductor is higher (lower) than the Fermi level in the metal. Charge transport that is limited by injection through a Schottky barrier is characterized by thermal excitation of charge carriers over the barrier, resulting in a thermally excited 
temperature dependence. The Mott-Schottky model is frequently applied also to organic semiconductors. For polymers, it is generally merged with the theory of back-diffusion of current through the Schottky barrier into the contact, as originally developed for inorganic disordered systems. In the highly-ordered organic single crystals, back-diffusion is expected not to play a role, and thus we consider it outside the scope of this work.

In preparations for contact fabrication on organic semiconductors, the MottSchottky model is generally used as a guideline for the choice of contact metal. The height of the Schottky barrier is given by the difference between the work function $\phi_{M}$ of the metal and the edge of the semiconductor band into which carriers are injected: for example, the barrier height for electrons is $\phi_{S}=\phi_{M}-\chi$ (see Fig. 2.7), where $\chi$ is the electron affinity. According to this rule, gold $\left(\phi_{M}=\right.$ $5.1 \mathrm{eV}$ ) is generally a good hole injector for organic semiconductors, because it forms low Schottky barriers. As an electron injector, lower work function material are more suitable, like calcium $\left(\phi_{M}=2.9 \mathrm{eV}\right)$. Experimental indication of the significance of Schottky barrier formation for organic semiconductor research is given by Ref. [51], which reports on the dependence of the hole injection efficiency of various metal/organic systems on the interfacial barrier height, estimated as the difference between the metal work function and the hole ionization potential in the organic material.

Several aspects can modify the Mott-Schottky-type of band bending at a metal/organic interface; two regularly mentioned examples are image-charge effects and the formation of an interface dipole layer. The image charge effect on the Schottky-barrier height is well-known from inorganic semiconductor physics, and therefore often extrapolated to organic semiconductors. A charge carrier in the semiconductor induces an image charge in the metal contact, thereby reducing its energy. These new energy states close to the metal/organic interface appear as a narrowing of the bandgap, and a significant lowering of the Schottky barrier is then expected [50]. Also interface dipoles influence the Schottky barrier height. Interfacial electric dipole layers have been reported to form spontaneously at metal/organic interfaces, inducing a vacuum level shift that changes the barrier height $[52,53]$. The nature of these spontaneous interface dipoles is yet unknown [54].

Another aspect that can have a major influence on the injection of charge carriers, although it is not often mentioned, is the presence of traps at the metal/organic interface that are induced by the contact fabrication [55]. Organic single crystals are very fragile, which poses a challenge for processing them into devices. The deposition of contacts on the crystal surface can damage the crystal locally, leading to an area in the crystal, near the metal/organic interface, 


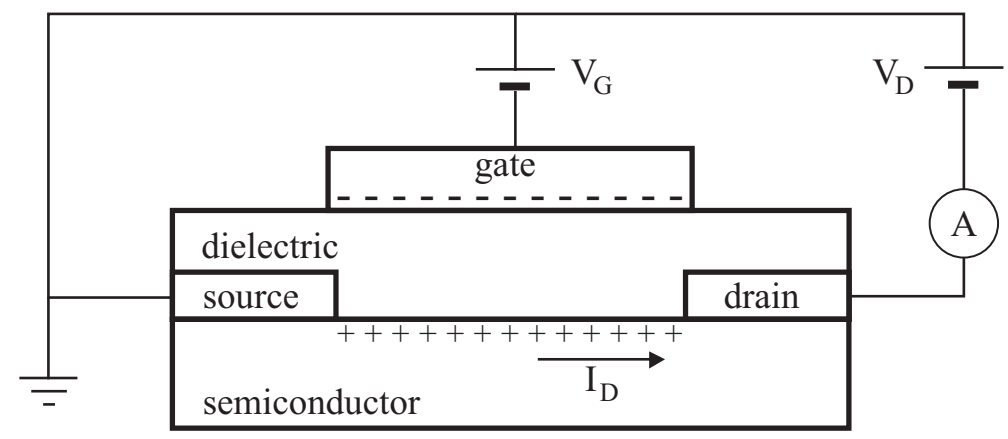

Figure 2.8: Schematic drawing of the FET geometry and the FET electronic circuitry. The voltage $V_{G}$ applied to the gate electrode determines the charge carrier density that is accumulated in the semiconductor, at the interface with the dielectric. The conductivity at the semiconductor/dielectric interface is then probed by applying a drain voltage $V_{D}$ and measuring the current $I_{D}$. The source contact is the reference electrode, connected to ground.

in which the density of traps is larger than in the bulk of the crystal. The influence of these surface traps on charge transport can be significant, as argued in Chapter 5.

\subsubsection{Field-effect transistors}

Metal-Oxide-Semiconductor Field-Effect Transistors (MOSFETs), based on inorganic semiconductors, have been extensively studied, because they are the workhorses of many integrated circuits [50]. It is unknown in how far the theory developed for MOSFETs can also be applied to organic FETs, considering the intrinsically different charge transport physics of organic and inorganic semiconductors. However, a microscopic description of charge transport in organic FETs is still lacking, and so far, the main aspects of experimental OFET behavior are very comparable to the operation of MOSFETs. Therefore, MOSFET theory is used as a starting point for the understanding of OFET behavior. In this Section, some basic aspects of MOSFET theory will be described, which will be used in later Chapters to interpret experimental results on OFETs.

A Field-Effect Transistor (FET) can in principle be considered as a parallel plate capacitor: the two capacitor plates are formed by a metal electrode, called the gate, and a semiconductor, which are separated from each other by an insulating layer, the gate dielectric (see Fig. 2.8). Two additional electrodes, the source and drain, contact the semiconductor and they can be used to probe the 


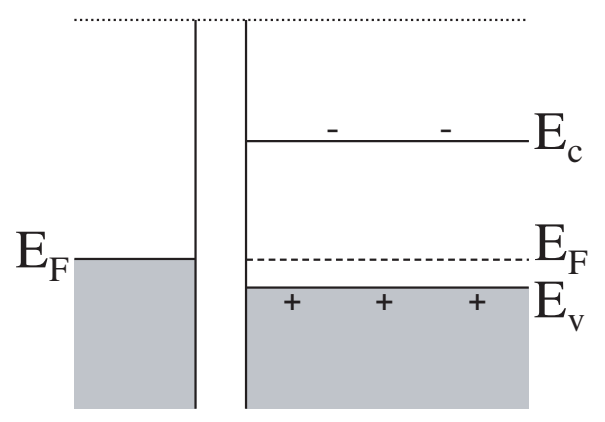

(a)

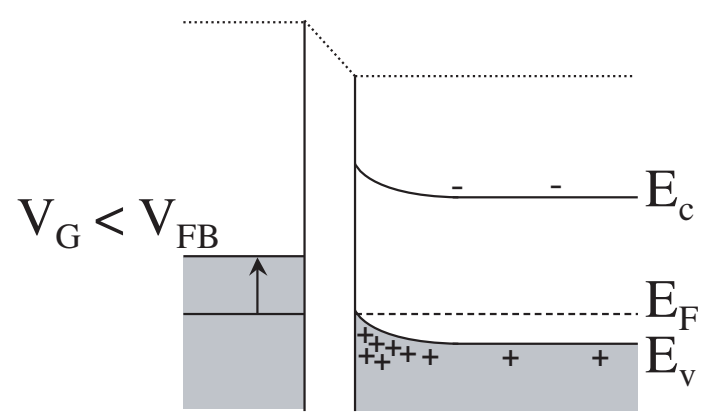

(b)

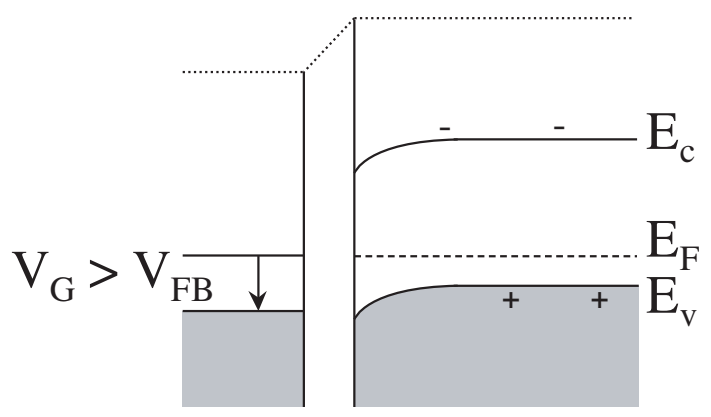

(c)

Figure 2.9: Energy-band diagrams for ideal metal-insulator-semiconductor (p-type) diode for the following cases: (a) flat-band condition, voltage applied to the metal is $V_{F B}$; (b) accumulation for applied voltage $V<V_{F B}$; (c) depletion for applied voltage $V>V_{F B}$.

conduction in the semiconductor. Applying a voltage between the gate and the source results in an electric field that attracts or repels charge carriers in the semiconducting FET channel, i.e. the area in the semiconductor situated in between source and drain and overlapping with the gate. Thus the conductivity in the channel, which depends on the charge carrier density (see Eq. 2.1), is controlled by the gate field that is applied. 
Charge transport in a FET is practically two-dimensional. Modification of the charge density takes place only in a thin layer of the semiconductor, close to the interface with the dielectric, i.e. the active layer. The bulk of the semiconductor is hardly or not at all affected by the gate-field, due to screening by the charge in the active layer. This can be understood in terms of band bending in the semiconductor, as visualized in Fig. 2.9 for a p-type semiconductor. When the gate voltage equals the flat-band voltage $V_{F B}$, which compensates for the Fermi level mismatch between the metal gate and the semiconductor, then there is no charge accumulation or depletion in the channel. For voltages lower than $V_{F B}$, positive charge carriers are accumulated in the channel, whereas increasing the voltage above $V_{F B}$ results in depletion of positive carriers. Bending of the bands towards their equilibrium position takes place within a few times the screening length from the semiconductor/dielectric interface, a distance that rapidly decreases as the charge density increases $[39,56]$.

The behavior of a unipolar FET is characterized by two regimes of operation [50]. In the saturation regime the source-drain current $I_{D}$ is quadratically dependent on the source-gate voltage $V_{G}$ :

$$
I_{D}=\frac{W}{2 L} \mu C_{i}\left(V_{G}-V_{T^{\prime}}\right)^{2}
$$

where $\mathrm{L}$ and $\mathrm{W}$ are the channel length and width, $C_{i}$ is the capacitance of the gate dielectric and $V_{T^{\prime}}$ is the threshold voltage. This equation is generally valid when $V_{G}-V_{T^{\prime}}$ is smaller than the source-drain voltage $V_{D}$. In the linear regime of operation $\left(V_{G}-V_{T} \gg V_{D}\right), I_{D}$ is linearly related to $V_{D}$ and $V_{G}$ :

$$
I_{D}=\frac{W}{L} \mu C_{i}\left(V_{G}-V_{T}\right) V_{D}
$$

where the threshold voltage $V_{T}$ is defined different from $V_{T^{\prime}}$. Both Eq. 2.8 and 2.9 contain the mobility of the semiconductor. In this Thesis, we use the measured linear regime to calculate the mobility, by rewriting Eq. 2.9 to:

$$
\mu=\frac{L}{W} \cdot \frac{1}{C_{i}} \cdot \frac{1}{V_{D}} \cdot \frac{\delta I_{D}}{\delta V_{G}}
$$

Eq. 2.9 is based on the assumption that all charge carriers induced by the transverse electric field above the threshold are mobile, and their density is given by: $n=C_{i}\left(V_{G}-V_{T}\right) / e$. This assumption has not been fully justified yet, although some justification is provided by observations of $V_{G}$-independent mobility and the mobility increase with cooling, as observed in several organic single-crystal FETs $[21,20]$. 
Next, we address some known differences between inorganic FETs and organic single-crystal FETs, that should be taken into account when applying the inorganic FET theory. A first dissimilarity is the interpretation of the threshold voltage. In standard inorganic FET theory, the threshold voltage $V_{T}$, defined as in Eq. 2.9, is given by the following formula:

$$
V_{T}=V_{F B}+2 \phi_{B}+\frac{\sqrt{4 \epsilon \epsilon_{0} e N_{A} \phi_{B}}}{C_{i}}
$$

where $\phi_{B}$ is the potential difference from the Fermi level to the middle of the bandgap, $\epsilon$ is the relative dielectric constant of the semiconductor and $N_{A}$ is the doping density. In organic FETs, the flat-band voltage $V_{F B}$ is generally small compared to the voltages that are applied (i.e. up to $\pm 100 \mathrm{~V}$ ), and since organic single crystals are not intentionally doped, both $\phi_{B}$ and $N_{A}$ are close to zero. Thus, the (for p-type conductivity) positive threshold voltage from Eq. 2.11 is expected to be negligible. In fact, a negative threshold voltage is rather expected, due to injection barriers at the source and drain contacts, and/or due to traps in the channel that have to be filled before a current of free charge carriers can flow. Podzorov et al. [20] have used the latter reasoning to estimated, from fourterminal measurements, the surface trap density in the channel with the simple formula: $N_{t}=\left(C_{i} V_{T}\right) / e$. However, for the OFETs fabricated by the electrostatic bonding of organic crystals, a relatively large positive threshold voltage $(10 \mathrm{~V}$ or more) is often observed. This behavior is illustrated in Fig. 2.10 for rubrene and for tetracene crystals bonded to the RIE pre-cleaned $\mathrm{SiO}_{2}$ surface [21], and similar behavior has been observed for pentacene [34]. The positive threshold has been also observed for the rubrene crystals bonded electrostatically to the surface of PDMS rubber stamps [16]. These observations suggest that the same microscopic mechanism responsible for electrostatic bonding might be responsible for inducing the built-in channel on the organic surface.

Another difference between organic semiconductor FETs and the inorganic FET theory as discussed in this Section, is the doping density: inorganic semiconductors are usually heavily doped, as opposed to organic single crystals, which are ultra-pure and undoped. Due to their large bandgap, the amount of free charge in the bulk of the organic single crystal is negligible. As a consequence, there is no depletion of free charge carriers from the active layer in the undoped semiconductor. Another interesting consequence is that charges that are accumulated in the active layer must be injected from the contacts, instead of being accumulated from the bulk of the semiconductor. The last point enhances the importance of the contacts in the formation of an accumulation layer and complicates the role of the contacts in the FET charge transport in general. A precise description or 


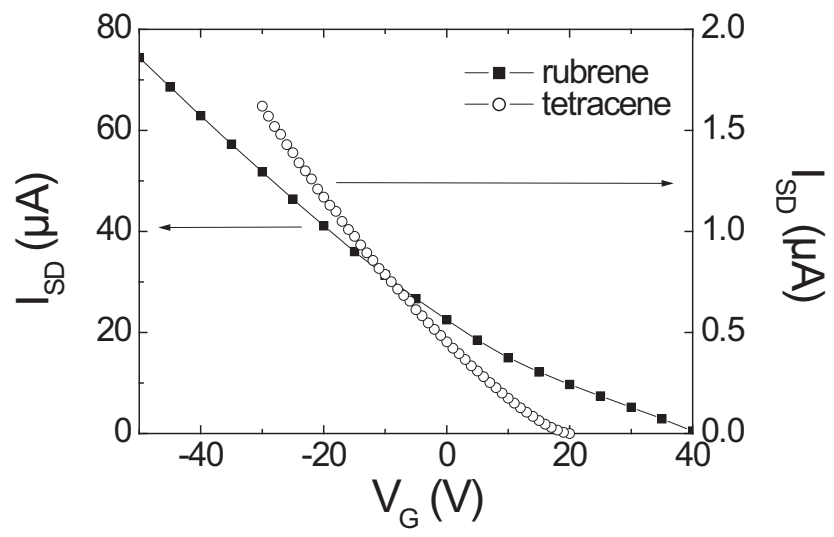

Figure 2.10: Gate-sweeps demonstrating a built-in channel for electrostatically bonded crystals, both for a tetracene (open circles) and for a rubrene (filled squares) single-crystal FET. The $W / L$ ratio is the same for the two devices, $W / L=0.14$.

model has not yet been developed.

In conclusion, in this Section we have discussed the basic theory of inorganic metal/semiconductor interfaces and inorganic MOSFETs that is used for the analysis of organic FET behavior. Also, we have touched upon a few known fundamental differences between the inorganic and organic semiconductors in interfaces and FETs. Using this as a starting point to increase the principle understanding of organic semiconductors and to identify the aspects that influence the organic FET performance, like we do in this Thesis, may in the future result in the development of a transport model that is fully dedicated to organic semiconductors.

\section{References}

[1] See, for example: www.wikipedia.org/wiki/Organic_compound

[2] E. A. Silinsh, and V. Čápek, Organic Molecular Crystals: Interaction, Localization, and Transport Phenomena (AIP Press, Woodbury, 1994)

[3] M. Pope, and C. E. Swenberg, Electronic Processes in Organic Crystals and Polymers, 2nd ed. (Oxford University Press, New York, 1999)

[4] R. D. Gould, Coord. Chem. Rev. 156, 237 (1996)

[5] M. -S. Liao, and S. Scheiner, J. Chem. Phys. 114, 9780 (2001)

[6] H. Sirringhaus, N. Tessler, and R. H. Friend, Science 280, 1741 (1998)

[7] I. H. Campbell, and D. L. Smith, Solid State Phys. 55,1 (2001) 
[8] E. J. Meijer, D. M. de Leeuw, S. Setayesh, E. van Veenendaal, B. H. Huisman, P. W. M. Blom, J. C. Hummelen, U. Scherf, and T. M. Klapwijk, Nature Mater. 2, 678 (2003)

[9] C. C. Mattheus, Polymorphism and Electronic Properties of Pentacene, Ph.D. thesis, ISBN 90-3671637-3 (PrintPartners Ipskamp B.V., Enschede, 2003)

[10] C. A. Hunter, and J. K. M. Sanders, J. Am. Chem. Soc. 112, 5525 (1990)

[11] C. R. Newman, C. D. Frisbie, D. A. da Silva Filho, J. -L. Brédas, P. C. Ewbank, and K. R. Mann, Chem. Mater. 16, 4436 (2004)

[12] R. C. Haddon, X. Chi, M. E. Itkis, J. E. Anthony, D. L. Eaton, T. Siegrist, C. C. Mattheus, and T. T. M. Palstra, J. Phys. Chem. B 106, 8288 (2002)

[13] Y. C. Cheng, R. J. Silbey, D. A. da Silva Filho, J. P. Calbert, J. Cornil, and J. -L. Brédas, J. Chem. Phys. 118, 3764 (2003)

[14] G. A. de Wijs, C. C. Mattheus, R. A. de Groot, and T. T. M. Palstra, Synth. Met. 139, 109 (2003)

[15] R. Farchioni, and G. Grosso, Organic electronic Materials (Springer-Verlag, Berlin, 2001)

[16] V. C. Sundar, J. Zaumseil, V. Podzorov, E. Menard, R. L. Willett, T. Someya, M. E. Gershenson, and J. A. Rogers, Science 303, 1644 (2004)

[17] Similarly, substituted side-groups on pentacene molecules influence the crystal structure, see J. E. Anthony, D. L. Eaton, and S. R. Parkin, Org. Lett. 4, 15 (2002)

[18] M. D. Curtis, J. Cao, and J. W. Kampf, J. Am. Chem. Soc. 126, 4318 (2004)

[19] D. A. da Silva Filho, E. -G. Kim, and J. -L. Brédas, Adv. Mater. 17, 1072 (2005)

[20] V. Podzorov, E. Menard, A. Borissov, V. Kiryukhin, J. A. Rogers, and M. E. Gershenson, Phys. Rev. Lett. 93, 086602 (2004)

[21] R. W. I. de Boer, T. M. Klapwijk, and A. F. Morpurgo, Appl. Phys. Lett. 83, 4345 (2003)

[22] C. Goldmann, S. Haas, C. Krellner, K. P. Pernstich, D. J. Gundlach, and B. Batlogg, J. Appl. Phys. 96, 2080 (2004)

[23] G. Horowitz, B. Bachet, A. Yassar, P. Lang, F. Demanze, J. -L. Fave, and F. Garnier, Chem. Mater. 7, 1337 (1995); T. Siegrist, R. M. Fleming, R. C. Haddon, R. A. Laudise, A. J. Lovinger, H. E. Katz, P. M. Bridenbaugh, and D. D. Davis, J. Mater. Res. 10, 2170 (1995) 
[24] C. C. Mattheus, A. B. Dros, J. Baas, G. T. Oostergetel, A. Meetsma, J. L. de Boer, and T. T. M. Palstra, Synth. Met. 138, 475 (2003); C. C. Mattheus, G. A. de Wijs, R. A. de Groot, and T. T. M. Palstra, J. Am. Chem. Soc. 125, $6323(2003)$

[25] U. Sondermann, A. Kutoglu, H. Bässler, J. Phys. Chem. 89, 1735 (1985)

[26] R. Silbey, J. Jortner, S. A. Rice, and M. T. Vala, J. Chem. Phys. 42, 733 (1965)

[27] G. Brocks, J. van den Brink, and A. F. Morpurgo, Phys. Rev. Lett. 93, 146405 (2004)

[28] W. Warta, and N. Karl, Phys. Rev. B 32, 1172 (1985)

[29] R. W. I. de Boer, M. E. Gershenson, A. F. Morpurgo, and V. Podzorov, Phys. Stat. Sol. 201, 1302 (2004)

[30] O. D. Jurchescu, J. Baas, and T. T. M. Palstra, Appl. Phys. Lett. 84, 3061 (2004)

[31] T. W. Kelley, P. F. Baude, C. Gerlach, D. E. Ender, D. Muyres, M. A. Haase, D. E. Vogel, and S. D. Theiss, Chem. Mater. 16, 4413 (2004)

[32] M. C. J. M. Vissenberg, and M. Matters, Phys. Rev. B 57, 12964 (1998)

[33] S. F. Nelson, Y. -Y. Lin, D. J. Gundlach, and T. N. Jackson, Appl. Phys. Lett. 72, 1854 (1998)

[34] J. Takeya, C. Goldmann, S. Haas, K. P. Pernstich, B. Ketterer, and B. Batlogg, J. Appl. Phys. 94, 5800 (2003)

[35] V. Y. Butko, J. C. Lashley and A. P. Ramirez, cond-mat/0405077 (2004)

[36] Note that the localization of charge carriers can have an intrinsic origin, like coupling to phonons (see Section 2.4.2), or an extrinsic origin (see Section 2.4.3).

[37] R. M. Glaeser, and R. S. Berry, J. Chem. Phys. 44, 3797 (1966)

[38] V. M. Kenkre, J. D. Andersen, D. H. Dunlap, and C. B. Duke, Phys. Rev. Lett. 62, 1165 (1989)

[39] J. Sinova, J. Schliemann, A. S. Nùñez, and A. H. MacDonald, Phys. Rev. Lett. 87, $226802(2001)$

[40] E. V. Tsiper, and Z. G. Soos, Phys. Rev. B 64, 195124 (2001)

[41] M. N. Bussac, J. D. Picon, and L. Zuppiroli, Europhys. Lett. 66, 392 (2004)

[42] T. Holstein, Ann. Phys. 8, 325 (1959) 
[43] R. W. Munn, and R. Silbey, J. Chem. Phys. 83, 1843 (1985)

[44] A. J. Millis, R. Mueller, and B. I. Shraiman, Phys. Rev. B 54, 5389 (1996)

[45] A. Girlando, M. Masino, A. Brillante, R. G. Della Valle, E. Venuti, Phys. Rev. B 66, 100507 (2002)

[46] K. Hannewald, and P. A. Bobbert, Appl. Phys. Lett. 85, 1535 (2004); K. Hannewald, V. M. Stojanović, J. M. T. Schellekens, P. A. Bobbert, G. Kresse, and J. Hafner, Phys. Rev. B 69, 075211 (2004)

[47] W. P. Su, J. R. Schrieffer, and A. J. Heeger, Phys. Rev. Lett. 42, 1698 (1979); W. P. Su, J. R. Schrieffer, and A. J. Heeger, Phys. Rev. B 22, 2099 (1980)

[48] K. Hannewald, and P. A. Bobbert, Phys. Rev. B 69, 075212 (2004)

[49] N. Karl, K. -H. Kraft, J. Marktanner, M. Münch, F. Schatz, R. Stehle, and H. -M. Uhde, J. Vac. Sci. Technol. A 17, 2318 (1999)

[50] S. M. Sze, Physics of Semiconductor Devices (Wiley, New York, 1981)

[51] M. Abkowitz, J. S. Facci, and J. Rehm, J. Appl. Phys. 83, 2670 (1998)

[52] H. Ishii, K. Sugiyama, E. Ito, and K. Seki, Adv. Mater. 11, 605 (1999)

[53] H. Peisert, M. Knupfer, T. Schwieger, J. M. Auerhammer, M. S. Golden, J. Fink, J. Appl. Phys. 91, 4872 (2002)

[54] An interface dipole layer can also be enforced by application of a SelfAssembled-Monolayer (SAM) to the metal before depositing the organic semiconductor, which leads to a controlled modification of the interface barrier height.

[55] C. D. Dimitrakopoulos, and D. J. Mascaro, IBM J. Res. \& Dev. 45,11 (2001)

[56] S. Wehrli, D. Poilblanc, and T. M. Rice, Eur. Phys. J. B 23, 345 (2001) 


\section{Chapter 3}

\section{From organic molecule to single-crystal transistor}

We describe our method to grow high-quality single crystals, and we discuss their characterization. In addition, we describe our technique to realize highquality single-crystal FETs by using electrostatic bonding of organic crystals on prefabricated silicon substrates. A description of other high-quality FET assembly techniques is also included. 


\subsection{Single-crystal growth and characterization}

The successful realization of Field-Effect Transistors (FETs) on the surface of Organic Molecular Crystals (OMCs) is an important milestone in the research of electronic transport in organic semiconductors. For the first time, it opens the opportunity to study the intrinsic behavior of charges at the organic surface, not limited by structural defects. Fabrication of organic single-crystal FETs comprises two main steps: the growth of an organic crystal with atomically-flat surface and preparation of the field-effect structure on this surface. In this Section we discuss both aspects, paying special attention to the analysis of advantages and limitations of different fabrication methods.

\subsubsection{Single-crystal growth}

The single crystals used for the fabrication of organic FETs have been grown from the vapor phase in a stream of transport gas, in horizontal reactors (quartz tube) $[1,2]$. In the Physical Vapor Transport (PVT) method, the starting material is placed in the hottest region of the reactor, and the crystal growth occurs within a narrow temperature range near its cold end (see Fig. 3.1). For better separation of larger and, presumably, purer crystals from the rest of re-deposited material along the tube, the temperature gradient should be sufficiently small (typically, $\left.2-5^{\circ} \mathrm{C} / \mathrm{cm}\right)$.

For the devices discussed in this Thesis, crystals were grown using ultra-highpurity Ar gas as a carrier agent. Other groups, however, report using other carrier gases, e.g., the best rubrene FETs fabricated so far have been grown in pure $\mathrm{H}_{2}$ $[3,4]$. It is unclear at present how exactly the transport gas affects the crystal quality; uncontrollable variations of the crystal quality might be caused by the residual water vapor and oxygen in the reactors. Photo-induced reactions with $\mathrm{O}_{2}$ are known for most organic molecules [5] and the products of these reactions can act as traps for charge carriers. To minimize possible photo-activated oxidation of organic material, the growth is performed in the dark.

Several factors affect the growth process and the quality of the crystals. Important parameters are, for instance, the temperature in the sublimation zone, $T_{\text {sblm }}$ and the gas flow rate. Many other factors can also play a role: e.g., acoustical vibrations of the reactor in the process of growth might affect the size, shape, and quality of the crystals. For each material and each reactor, the optimal parameters have to be determined empirically. At least in one case (the rubrenebased FETs [4]), it has been verified that the slower the growth process, the higher the field-effect mobility. For this reason, the temperature of sublimated organic 
material was chosen close to the sublimation threshold. The crystal growth in this regime proceeds by the flow of steps at a very low rate $\left(\leq 5 \cdot 10^{-7} \mathrm{~cm} / \mathrm{s}\right.$ in the direction perpendicular to the $a b$-plane), and results in a flat surface with a low density of growth steps [6]. As an example, sublimation of $\sim 200 \mathrm{mg}$ of tetracene starting material takes 24 hours at $T_{\text {sblm }}=300^{\circ} \mathrm{C}$.

Another important parameter is the purity of the starting material. As the crystal growth process also results in the chemical purification of the material, several re-growth cycles may be required for improving the field-effect mobility, with the grown crystals used as the starting material for the subsequent re-growth. The number of required re-growth cycles depends strongly on the purity of the starting material. Figure 3.2 illustrates the need for several re-growth cycles in the process of the growth of tetracene crystals. Despite the nominal $98 \%$ purity of the starting tetracene (Sigma-Aldrich), a large amount of residue left in the sublimation zone after the first growth cycle is clearly visible (Fig. 3.2b); this residue is not present at the end of the second growth cycle (Fig. 3.2c). Clearly, the better purity of the starting material, the fewer re-growth cycles are required for a high FET mobility.

Most of the organic crystals grown by the physical vapor transport are shaped as elongated "needles" or thin platelets (see Fig. 3.3, 3.4 and 3.5). The crystal shape is controlled by the anisotropy of inter-molecular interactions: for many materials, a larger crystal dimension corresponds to the direction of the strongest interactions and, presumably, the strongest overlap between $\pi$ orbitals of adjacent molecules. For this reason, the direction of the fastest growth of needle-like rubrene crystals coincides with the direction of the highest mobility of fieldinduced carriers. For platelet-like crystals, the larger facets are parallel to the $a b$-plane. Typical in-plane dimensions range from a few square millimeters for rubrene to several square centimeters in the case of anthracene. The crystal thickness also varies over a wide range and, in most cases, can be controlled by stopping the growth process at an early stage. For example, the thickness of the tetracene crystals grown for 24 hours ranges between $\sim 10 \mu \mathrm{m}$ and $\sim 200 \mu \mathrm{m}[7]$, but it is possible to harvest several crystals of sub-micron thickness by stopping the growth process after $\sim 30$ minutes.

\subsubsection{Crystal characterization}

To understand better the effect of different factors on the crystal growth, a thorough characterization of the crystal properties is needed. Note, however, that many experiments provide information on the crystal properties that is only indirectly related to the performance of the organic single-crystal FETs. For exam- 

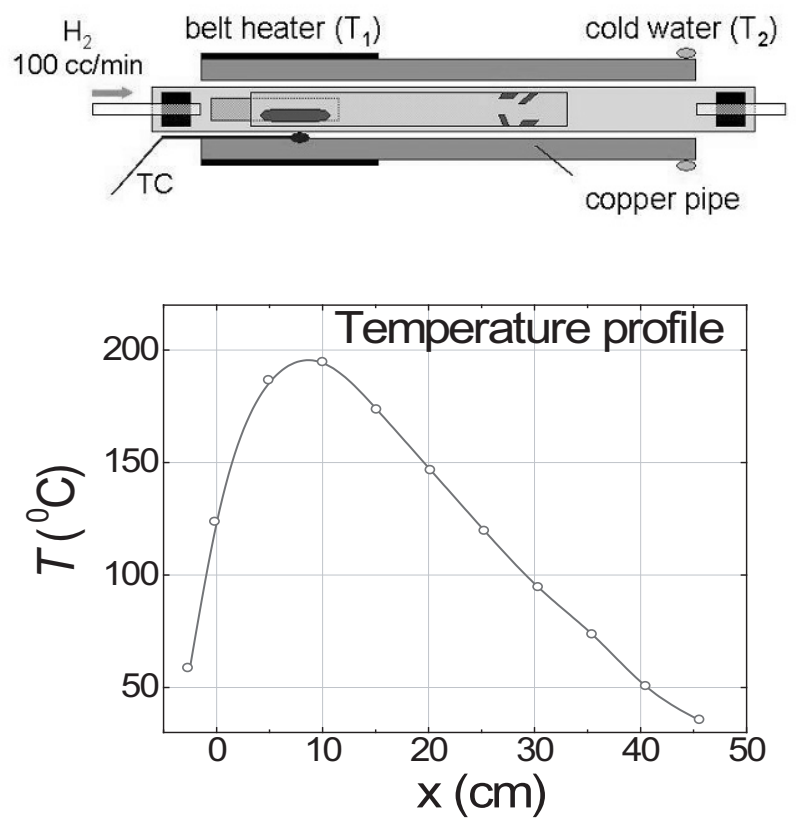

Figure 3.1: Schematic overview of crystal growth system. Organic material sublimes at temperature $T_{1}$, is transported through the system by the carrier gas and recrystallizes in the cooler end of the reactor. Heavy impurities (with a vapor pressure lower that that of the pure organic compound) remain at the position of the source material. Light impurities (with a vapor pressure higher than that of the pure organic compound) condense at a lower temperature, i.e. at a different position from where the crystals grow. Therefore, the crystal growth process also results in the purification of the material.

ple, the x-ray analysis of organic crystals, though necessary for identification of the crystal structure and orientation of the crystallographic axes, is insufficiently sensitive for detection of a minute concentration of defects that might severely limit the field-effect mobility at low temperatures. Similarly, the time-of-flight experiments, although useful in assessing the quality of the bulk of organic crystals, are not sensitive to the surface defects that limit the OFET performance. The crystals in this Thesis have been characterized by optical microscopy and by electrical characterization.

Inspection of crystals under an optical microscope in the polarized light provides a fast and useful analysis of the crystalline domain structure. Visualization of domains is possible because crystals of most organic conjugated materials are birefringent [8]. This allows us to select single crystals to be used for transport experiments by choosing those samples that become uniformly dark when changing the orientation of cross-polarizers. Optical inspection also enables detection of a 


\begin{tabular}{ccc} 
source & purified crystals & impurities \\
\hline & $\longrightarrow$ & $\longrightarrow$
\end{tabular}

(a)

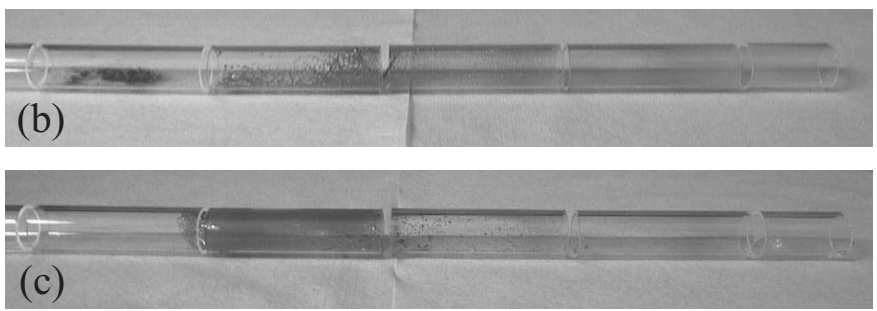

Figure 3.2: (a) Schematic overview of physical vapor growth setup indicating the position of source material, newly-grown purified crystals and impurities. (b) Result after first regrowth of as-purchased organic material. Purified crystals are visible in the middle; the dark residue present where the source material initially was and the material visible on the right of the purified crystals are due impurities. In reality, also the difference in color helps to distinguish between purified crystals and impurities. (c) At the end of the second regrowth no dark residue is present at the position of the source material, which demonstrate the purifying effect of the growth process.

thickness gradient or stress in crystals. Both phenomena cause the appearance of interference fringes, where in the former the orientation of the fringes is related to a specific crystallographic direction, and in the latter it is not. This technique simplifies the process of selection of single crystals for transport measurements.

For each different organic molecule, a selection of crystals was used for electrical characterization. Two techniques were used: Time-of-Flight (TOF) and Space-Charge Limited Current (SCLC) measurements. The techniques, and the experimental results on tetracene single crystals, are discussed in Chapter 4. Although electrical characterization is performed only on a selection of crystals, the results represent the quality of all crystals of the same material that were grown with PVT under the same conditions. More generally, it proves that the PVT growth is a useful technique for the fabrication of organic single-crystals with a low density of chemical and structural defects.

\subsection{Single-crystal FET fabrication techniques}

Fabrication of the field-effect structure on the surface of organic crystals poses a challenge, because many conventional fabrication processes irreversibly damage the surface of van der Waals bonded organic crystals by disrupting molecular 


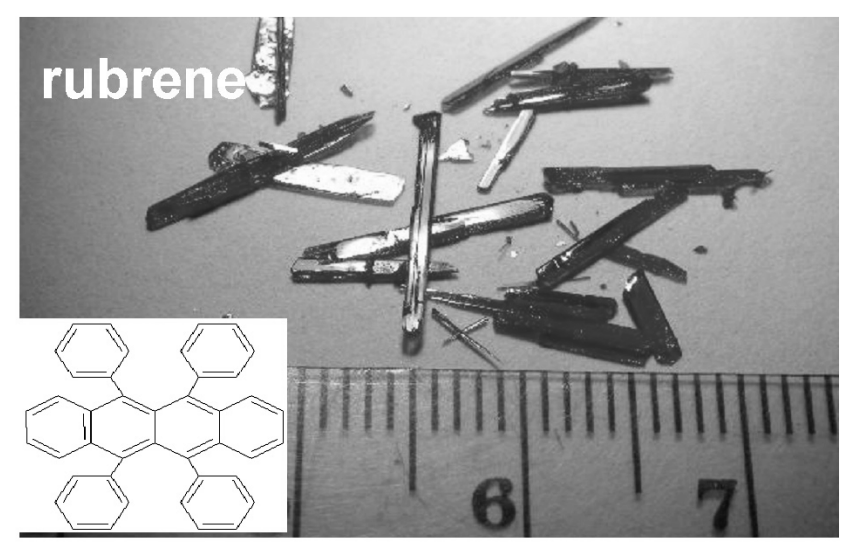

Figure 3.3: Result of rubrene crystal growth. Most of the organic crystals grown by the physical vapor transport are shaped as elongated "needles" or thin platelets.

order, generating interfacial trapping sites, and creating barriers to charge injection. Up to date, two techniques for organic single-crystal FET fabrication have been successfully used: (a) electrostatic "bonding" of an organic crystal to a prefabricated source/drain/gate structure, and (b) direct deposition of the contacts and gate insulator onto the crystal surface. Each of these techniques is discussed below. The different fabrication methods are utilized in Chapter 9 for a comparison of OFETs with different dielectrics, to investigate fundamental aspects that influence the OFET transport properties. The technique used more frequently in the rest of this Thesis is electrostatic bonding to a prefabricated silicon wafer, because of its compatibility with the use of high- $\epsilon$ dielectrics. This allows the accumulation of a large carrier density in OFETs, approaching one carrier per molecule, of which experimental results will be discussed in Chapters 8 and 9 .

\subsubsection{Electrostatic bonding technique}

In this approach, the transistor circuitry (both gate and source/drain electrodes) is fabricated on a separate substrate, which, at the final fabrication stage, is electrostatically bonded to the surface of an organic crystal. The technological processes vary depending on the type of a substrate for the transistor circuitry. Two kinds of substrates have been used: conventional silicon wafers $[9,10,11]$, and flexible elastomer substrates (the so-called rubber stamps) [12]. 


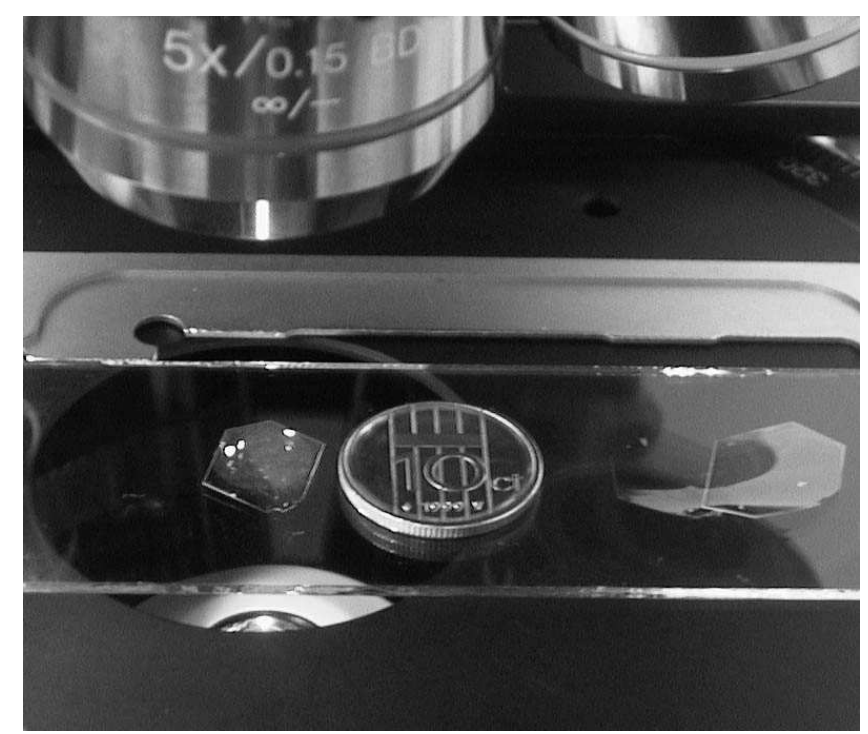

Figure 3.4: Two platelet-shaped anthracene single-crystals grown by physical vapor transport. The crystals are transparent and colorless. The left crystal is illuminated by UV light, and fluoresces in the blue.

\section{Source/drain/gate structures on $\mathrm{Si}$ substrates}

In this method, the source/drain/gate structure is fabricated on the surface of a heavily doped (n-type or p-type) Si wafer, covered with a layer of thermally grown $\mathrm{SiO}_{2}$ (typically, $0.2 \mu \mathrm{m}$ thick). The conducting $\mathrm{Si}$ wafer serves as the gate electrode, and the $\mathrm{SiO}_{2}$ layer plays the role of the gate insulator. The source and drain gold contacts are deposited on top of the $\mathrm{SiO}_{2}$ layer, and, as a final step, a sufficiently thin OMC crystal is electrostatically bonded to the source/drain/gate structure. We find that the Reactive Ion Etching (RIE) of the contact/ $\mathrm{SiO}_{2}$ surface in the oxygen plasma prior to the OMC bonding improves significantly the characteristics of tetracene FETs: the RIE cleaning reduces the spread of mobilities, the field-effect threshold voltage, and the hysteresis of transfer characteristics. The RIE cleaning also significantly improves adhesion of freshly grown tetracene crystals to $\mathrm{SiO}_{2}$ surface. The technique works best for very thin crystals ( $\leq 1 \mu \mathrm{m}$ thick) that adhere spontaneously to the substrate, but it can also be applied (with a lower success yield) to much thicker crystals by gently pressing on the crystal to assist the adhesion process [13]. Fig. 3.6 shows a picture of a rubrene FET fabricated with the technique of electrostatic adhesion to $\mathrm{SiO}_{2}$. The technique is further discussed in Chapter 6, including results on tetracene single-crystal FETs fabricated with this method. 


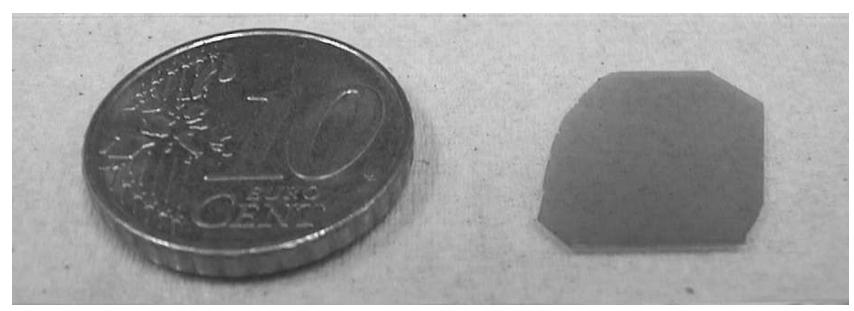

Figure 3.5: A $\mathrm{cm}^{2}$-sized platelet-shaped tetracene single-crystal grown by physical vapor transport.

\section{Source/drain/gate structures on flexible substrates}

In this approach, an organic crystals is bonded to a flexible elastomer substrate (polydimethylsiloxane, or PDMS), on top of which the FET circuitry is fabricated [12]. The fabrication starts with casting and curing of a prepolymer of PDMS against a pattern of photoresist on a Si wafer, thus defining an elastomeric "stamp" with an imprint that is complementary to the resist pattern. Coating this stamp with metal generates electrically isolated electrodes on the raised and recessed regions. The raised regions act as source- and drain electrodes, and the recessed region is the gate electrode. The final assembly of the devices, similar to the Si-based technique, consists of positioning of the OMC crystal on the stamp surface, and applying a gentle pressure to one edge of the crystal. Van der Waals forces then spontaneously cause a "wetting" front to proceed across the crystal surface. The crystal adheres to the source- and drain-electrodes and spans the distance between these electrodes, so that the thin (air or vacuum) space between the crystal and the gate-electrode plays the role of the gate dielectric.

\section{Advantages versus disadvantages}

The main advantage of both Si- and PDMS-based stamp technique is obvious: it eliminates the need for deposition of metals and dielectrics directly onto a very vulnerable organic surface. Since these techniques exploit the technologies well-developed in electronic industry, the dimension of the circuitry can be easily reduced, if desirable, well in the sub-micron range: specifically, a very small source/drain contact separation can be achieved by using electron-beam lithography. Interestingly, simple adhesion of organic crystals to metallic surface results in contacts with good electrical properties. This has been demonstrated for both Si- and PDMS- based stamps by comparing the results of two- and four-probe measurements $[14,15]$.

Although its simplicity makes electrostatic bonding particularly appealing for the fabrication of organic single-crystal FETs, this technique also suffers from a 
number of limitations. For instance, the choice of metals for the source and drain contacts in the electrostatic "bonding" technique is limited by noble metals, since other materials are easily oxidized in air. The channel width is not well defined (unless it is limited by the crystal dimensions or by patterning the gate electrode), because the conduction channel is formed over the whole area of overlap between the OMC crystal and the Si wafer. Finally, another potential problem of the Siand PDMS stamps might be the mismatch between the coefficients of thermal expansion for the stamp and the organic crystal. Upon changing the temperature, this mismatch might cause a mechanical stress and formation of defects in the crystal. Since the surface defects can trap the field-induced charge, this might result in deterioration of the low-temperature OFET characteristics. This is an important issue that requires further studies.

\section{Organic FETs with high- $\epsilon$ dielectrics}

The electrostatic bonding technique for OFETs is compatible with the use of high- $\epsilon$ dielectrics as gate insulators. The fabrication process of FETs with high- $\epsilon$ dielectrics is similar to the aforementioned Si-based technique, with $\mathrm{SiO}_{2}$ replaced by a high- $\epsilon$ dielectric that is sputtered onto a heavily doped silicon substrate. The use of high- $\epsilon$ dielectrics allows the accumulation of a large carrier density in OFETs. Particularly interesting is the possibility of reaching a surface charge density of the order of $1 \cdot 10^{14}$ carriers $/ \mathrm{cm}^{2}$, which corresponds to approximately one charge carrier per molecule (this estimate assumes that all the charges are accumulated in a single molecular layer, as it is expected from calculations of the screening length $[16,17])$. Indeed, the maximum surface charge density is $Q=\epsilon \epsilon_{0} E_{b d}$, where $\epsilon$ is the relative dielectric constant of the gate dielectric and $E_{b d}$ is its breakdown field. For typical high- $\epsilon$ dielectrics, such as $\mathrm{Ta}_{2} \mathrm{O}_{5}$ or $\mathrm{ZrO}_{2}$, $\epsilon=25$ and $E_{b d}>6 \mathrm{MV} / \mathrm{cm}$, and the resulting charge density at the breakdown is $10^{14}$ carriers $/ \mathrm{cm}^{2}$. Many novel high- $\epsilon$ materials hold the promise of even higher charge densities [18, 19].

\subsection{2 "Direct" FET fabrication on the crystal surface}

The "direct" fabrication of the single-crystal OFETs, in which a free-standing $\mathrm{OMC}$ is used as the substrate for subsequent deposition of the contacts and gate dielectric, is not trivial, because the organic crystals are incompatible with the standard processes of thin-film deposition/patterning. For example, after many unsuccessful attempts, it became clear that sputtering of $\mathrm{Al}_{2} \mathrm{O}_{3}$, as well as other dielectrics, onto the surface of organic molecular crystals unavoidably results in 
a very high density of traps and prohibitively high field-effect threshold: the field effect is completely suppressed even if the organic crystals are positioned in the shadow region of the vacuum chamber, where the deposition rate is zero. Presumably, the OMC surface is damaged by high-energy particles in the plasma. The attempts to shield the surface from high-energy charged particles by electrostatic deflection does not improve the situation.

Fabrication of the field-effect structures based on single crystals of organic semiconductors became possible after several innovations have been introduced both for the source/drain fabrication and for the gate dielectric deposition $[3,4]$.

\section{Source/drain contacts}

Different routes have been followed for the fabrication of source/drain contacts on the surface of organic crystals. The simplest (but also the crudest) is the "manual" application of a conducting paste. Disadvantages of this method are that it is difficult to prepare small and nicely-shaped contacts on OMC surfaces, and that it often results in formation of defects (traps) at the contact/organic interface (see Chapters 4 and 5). The thermal deposition of metals through a shadow mask is a more versatile method. However, the thermal load on the crystal surface in the deposition process (mostly because of radiation from the evaporation boat) has to be painstakingly minimized: deposition might generate traps at the metal/organic interface, or even result in the OMC sublimation. Following a careful contact evaporation procedure, the authors of Ref. [4] have succeeded in fabricating high-quality OFETs on the surface of several organic crystals (rubrene, TCNQ, pentacene).

\section{Parylene as a gate dielectric}

The breakthrough in the "direct" fabrication of free-standing single-crystal OFETs came with using thin polymer films of parylene as a gate-dielectric material [3]. This material with the dielectric constant $\epsilon=2.65$ forms transparent pinhole-free conformal coatings, impenetrable to oxygen and water, with excellent mechanical and dielectric properties: the breakdown electric field could be as high as $\sim 10$ $\mathrm{MV} / \mathrm{cm}$ for the thickness $0.1 \mu \mathrm{m}$.

Parylene is deposited in a reactor with three temperature zones. Prior to the deposition, the reactor (quartz tube) is evacuated to a pressure of $\sim 0.1$ mbar. The dimer para-xylylene (generic name, parylene) is vaporized in the vaporization zone at $\sim 100^{\circ} \mathrm{C}$, decomposes into monomers in the pyrolysis zone at $\sim 700^{\circ} \mathrm{C}$, and polymerizes in the deposition zone (the sample location) at room temperature. Parylene is deposited onto the OMC crystals with pre-fabricated 


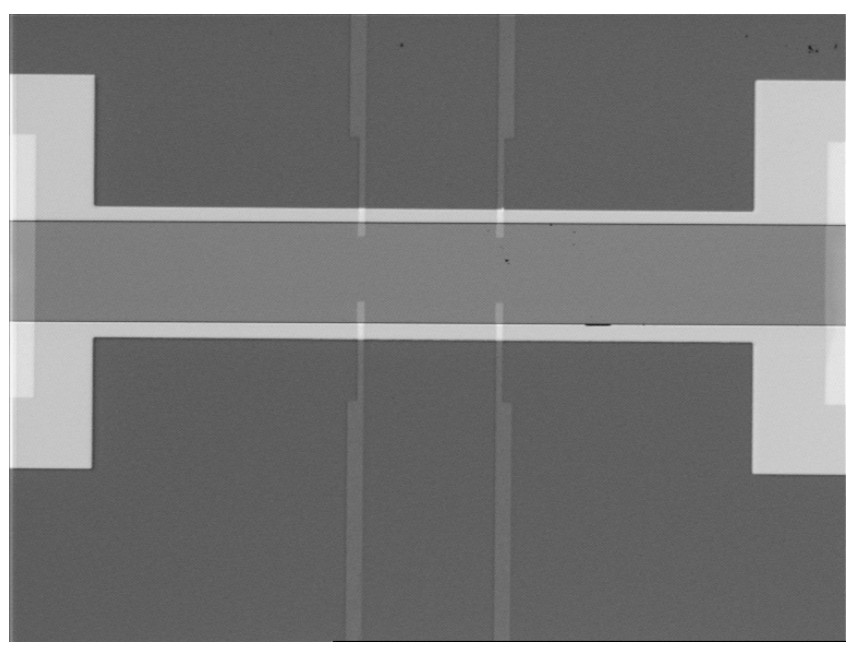

Figure 3.6: Optical microscope picture of a single-crystal rubrene FET, fabricated by electrostatic bonding. The crystal, which has a rectangular shape, overlaps with the source and drain contacts (at the left and right edge of the picture) and with four small contacts in the center, used to perform 4-probe electrical measurements. The purple area consists of a $\mathrm{Ta}_{2} \mathrm{O}_{5}$ layer sputtered on top of the substrate prior to the crystal bonding, which, for wider crystals, serves to confine the electrically active region of the FET.

source and drain contacts, and the device is completed by depositing a gateelectrode on top of the parylene layer.

There are several important advantages of using parylene as the gate dielectric: (a) it can be deposited while the crystal remain at room temperature, (b) being chemically inert, it does not react with OMCs, and (c) the parylene/OMC interface has a low density of surface states. Apart from that, parylene is a carbon-based polymer, and its thermal expansion coefficient is likely to be close to that of most organic crystals (but that remains to be tested). As it has already been emphasized above, different thermal expansion/contraction of the crystal and gate dielectric might result in the stress-induced carrier trapping. In this regard, the use of parylene is particularly promising for the operation of OFETs at low temperature.

\section{References}

[1] Ch. Kloc, P. G. Simpkins, T. Siegrist, and R. A. Laudise, J. Cryst. Growth 182, 416 (1997)

[2] R. A. Laudise, Ch. Kloc, P. G. Simpkins, and T. Siegrist, J. Crystal Growth 
187, $449(1998)$

[3] V. Podzorov, V. M. Pudalov, and M. E. Gershenson, Appl. Phys. Lett. 82, 1739 (2003)

[4] V. Podzorov, S. E. Sysoev, E. Loginova, V. M. Pudalov, and M. E. Gershenson, Appl. Phys. Lett. 83, 3504 (2003)

[5] R. Dabestani, M. Nelson, and M. E. Sigman, Photochem. Photobiol. 64, 80 (1996)

[6] R. A. Laudise, The Growth of Single Crystals (Englewood Cliffs, N. J., Prentice-Hall, 1970)

[7] R. W. I. de Boer, M. Jochemsen, T. M. Klapwijk, A. F. Morpurgo, J. Niemax, A. K. Tripathi, and J. Pflaum, J. Appl. Phys. 95, 1196 (2004)

[8] J. Vrijmoeth, R. W. Stok, R. Veldman, W. A. Schoonveld, and T. M. Klapwijk, J. Appl. Phys. 83,3816 (1998)

[9] R. W. I. de Boer, T. M. Klapwijk, and A. F. Morpurgo, Appl. Phys. Lett. 83, 4345 (2003)

[10] J. Takeya, C. Goldmann, S. Haas, K. P. Pernstich, B. Ketterer, and B. Batlogg, J. Appl. Phys. 94, 5800 (2003)

[11] M. Ichikawa, H. Yanagi, Y. Shimizu, S. Hotta, N. Suganuma, T. Koyama, and Y. Taniguchi, Adv. Mater. 14, 1272 (2002)

[12] J. Zaumseil, T. Someya, Z. N. Bao, Y. L. Loo, R. Cirelli, and J. A. Rogers, Appl. Phys. Lett. 82, 793 (2003)

[13] O. Jurchescu, and T. T. M. Palstra, private communication

[14] I. N. Hulea, unpublished results

[15] C. L. Mulder, Elastomeric Air-Gap Field-Effect Transistors at the Surface of Organic Single Crystals, master thesis (unpublished, Delft University of Technology, 2005)

[16] J. Sinova, J. Schliemann, A. S. Nùñez, and A. H. MacDonald, Phys. Rev. Lett. 87, 226802 (2001)

[17] S. Wehrli, D. Poilblanc, and T. M. Rice, Eur. Phys. J. B 23, 345 (2001)

[18] R. B. van Dover, L. D. Schneemeyer, and R. M. Fleming, Nature 392, 162 (1998)

[19] W. Ren, S. Trolier-McKinstry, C. A. Randall, and T. R. Shrout, J. Appl. Phys. 89, 767 (2001) 


\title{
Chapter 4
}

\section{Space-charge limited transport and time-of-flight measurements in tetracene single crystals}

\begin{abstract}
We report on a systematic study of electronic transport in tetracene single crystals by means of space-charge limited current spectroscopy and time-of-flight measurements. Both $I-V$ and time-of-flight measurements show that the roomtemperature effective hole-mobility reaches values close to $\mu \simeq 1 \mathrm{~cm}^{2} / \mathrm{Vs}$ and show that, within a range of temperatures, the mobility increases with decreasing temperature. The experimental results further allow the characterization of different aspects of the tetracene crystals. In particular, the effects of both deep and shallow traps are clearly visible and can be used to estimate their densities and characteristic energies. The results presented in this Chapter show that the combination of $I-V$ measurements and time-of-flight spectroscopy is very effective in characterizing several different aspects of electronic transport through organic crystals.
\end{abstract}

This Chapter has been published as R. W. I. de Boer, M. Jochemsen, T. M. Klapwijk, A. F. Morpurgo, J. Niemax, A. K. Tripathi, and J. Pflaum, J. Appl. Phys. 95, 1196 (2004) 


\subsection{Introduction}

Organic devices for electronic applications are usually based on thin film technology $[1,2]$. This is particularly advantageous, as thin films of organic molecules and polymers can be manufactured easily and cheaply in different ways. Over the past few years, an intense research effort has resulted in rapid improvement of the manufacturing processes, which has allowed the commercialization of products based on organic devices, i.e. organic electronics.

In spite of the rapid progress that has taken place on the applied side, the rather low chemical and structural purity of the thin films used in device fabrication has so far prevented a systematic study of the intrinsic electronic properties of organic semiconductors. That is because for these films, it is the defects that determine the behavior observed experimentally [3]. As a consequence, our basic understanding of the electronic properties of organic materials is still limited.

Improved chemical and structural purity in organic conductors can be obtained by using single crystals of small conjugated organic molecules. Electronic transport through single crystals of different organic molecules has been investigated in the past by means of Time-of-Flight (TOF) measurements $[4,5]$. It has been found that the hole mobility is approximately $1 \mathrm{~cm}^{2} / \mathrm{Vs}$ at room temperature, increasing up to values in excess of $100 \mathrm{~cm}^{2} / \mathrm{Vs}$ with decreasing temperature. Since these observations have been reported only in the highest purity crystals, it is believed that this is the intrinsic behavior of charge carrier mobility in organic conductors. So far however, this intrinsic behavior has never been observed in conventional DC transport measurements.

In this Chapter we report an experimental study of DC transport through tetracene single crystal and show that our results exhibit some of the features expected for the intrinsic behavior of organic conductors. Our investigations are based on the study of the current-voltage $(I-V)$ measurements in the spacecharge limited current regime and on their comparison to TOF measurements performed on identically grown crystals. As we will show, we find overall agreement between the results obtained with the two different methods. In particular, both $I-V$ and TOF measurements show that the room-temperature effective hole-mobility reaches values close to $\mu \simeq 1 \mathrm{~cm}^{2} / \mathrm{Vs}$. Both measurement techniques also show that, within a range of temperatures, the mobility increases with decreasing temperature. For the best samples probed by $I-V$ measurements this range extends down to approximately $200 \mathrm{~K}$, below which a structural phase transition known to occur in tetracene causes a sudden drop of the mobility. The experimental results further allow the characterization of different aspects of the tetracene crystals. In particular, we observe the effect of both deep and 
shallow traps [6]. For the former, the measurement of the $I-V$ characteristics give us an upper bound on their bulk density $\left(N_{t}^{d}<5 \cdot 10^{13} \mathrm{~cm}^{-3}\right)$ and an estimate of their depth ( $E_{t}^{d} \approx 700 \mathrm{meV}$ relative to the edge of the valence band). The concentration of shallow traps is substantially larger and only a very rough estimate can be obtained from TOF experiments.

The Chapter is organized as follows. We first describe the most important aspects of the tetracene crystals growth and of the sample preparation (Section 4.2). The behavior of the measured $I-V$ curves is presented in Section 4.3. In this Section we also discuss the basic aspects of the theoretical concepts necessary to interpret the experimental data. Section 4.4 is devoted to TOF experiments. Finally, in Section 4.5 we summarize and compare the outcome of TOF and DC transport measurements and we present our conclusions.

\subsection{Crystal growth and sample preparation}

Single tetracene crystals are grown by means of physical vapor deposition in a temperature gradient (see Fig. 3.1), in the presence of a stream of carrier gas, using a set-up similar to that described in Ref. [7]. The source material for the first crystal growth is $98 \%$ pure tetracene purchased from Sigma-Aldrich. Crystals grown from as-purchased tetracene are used as source material for a subsequent growth process, as an extra purification step (see Fig. 3.2).

Tetracene crystals grown by physical vapor deposition are large platelets, with surfaces parallel to the $a b$-plane. Typical dimensions range from few square millimeters to $1 \times 1 \mathrm{~cm}^{2}$ or larger. For crystals grown by letting the growth process proceed overnight, the typical crystal thickness ranges between $\sim 10 \mu \mathrm{m}$ and $\sim 200 \mu \mathrm{m}$. We have performed X-ray structural study on a few of our thickest crystals and found a structure consistent with known literature data $[8,9]$. As discussed in Chapter 3, multiply regrown crystals are inspected under an optical microscope with polarized light, to simplify the process of selection of single crystals for transport measurements.

All $I-V$ and TOF measurements discussed in this Chapter have been performed in the direction perpendicular to the crystal $a b$-plane, using electrical contacts on the two opposite faces of crystals. Electrodes for $I-V$ measurements are fabricated by connecting gold wires to the crystal surface using a two-component, solvent-free silver epoxy, so that the epoxy is in direct contact with the crystal. The contact area is measured under the microscope and it is typically of the order of $0.1 \mathrm{~mm}^{2}$. We use silver epoxy CW2400 (Circuitworks), which hardens at room temperature in a few hours. This contact fabrication method is very quick and 
it was chosen because it allows the investigation of many samples in relatively short time. Other types of contacts were tested as well, i.e. metal evaporated contacts and colloidal graphite paint contacts, but they did not result in any improvement of the observed electrical properties. For TOF measurements we used silver electrodes prepared by thermal evaporation through a shadow mask.

\subsection{DC transport through tetracene single crys- tals}

In this Section we discuss the results obtained by studying the $I-V$ characteristics of approximately 100 tetracene single crystals. We have found that the measured $I-V$ curves exhibit large sample-to-sample deviations so that particular care has to be taken in the interpretation of the experimental data. For this reason, we first discuss how the charge carrier mobility can be estimated using concepts of space-charge limited current theory of general validity, i.e. not sensitive to the detailed behavior of our samples. After having presented the experimental results in terms of the concept previously introduced, we discuss the role of deep traps present in the bulk of the crystals and at the metal/organic contact interface. We argue that the latter provide the most likely explanation of the large sample to sample variation observed in the measured $I-V$ characteristics.

\subsubsection{Estimate of the carrier mobility}

Since the band-gap of tetracene is approximately $E_{g} \simeq 3 \mathrm{eV}$ [10], high-purity tetracene crystals containing a negligible amount of dopants essentially behave as insulators. It is still possible to pass a current through tetracene crystals by applying a sufficiently large voltage, which acts both to transfer charge from the electrodes into the crystal and to accelerate that charge. When the charge injected from the contacts is larger than the charge present in the material in equilibrium the $I-V$ characteristics become non-linear and transport is said to occur in the space-charge limited regime [11].

For materials in which current is carried by only one carrier type (holes in our tetracene crystals; see Section 4.4.2), there exists an upper limit to the current that can be carried in the space-charge limited regime. This is due to electrostatics that, at any given voltage $V$, fixes the maximum amount of charge that can be injected into the material. For the geometry used in our experiments, the resulting maximum current that can flow in the space-charge limited regime in 
the presence of an applied voltage $V$ is [11]:

$$
I=A \frac{9 \epsilon \epsilon_{0} \mu V^{2}}{8 L^{3}}
$$

with $A$ and $L$ respectively electrode area and separation and $\epsilon$ relative dielectric constant of the material ( $\epsilon \simeq 3$ for tetracene). This upper limit is intrinsic and it is not sensitive to any of the specific sample details that determine the shape of the $I-V$ curves. For any applied voltage, contact effects, defects or traps can only reduce the current below the value given by Eq. 4.1 .

We use these considerations to obtain experimentally a lower limit $\mu_{\min }$ for the mobility of charge carrier, by measuring the current $I$ induced by a voltage $V$ and by "inverting" Eq. 4.1. We obtain:

$$
\mu_{\min }=\frac{8 I L^{3}}{9 A \epsilon \epsilon_{0} V^{2}}
$$

If $\mu_{\text {min }}$ is very low - e.g., at room temperature, much lower than the intrinsic value $1 \mathrm{~cm}^{2} /$ Vs typical of organic semiconductors - this approach does not provide any useful information. However, if the value of $\mu_{\min }$ is close to $1 \mathrm{~cm}^{2} / \mathrm{Vs}$, this analysis indicates that the quality of the crystal is high (since the intrinsic mobility $\mu>$ $\left.\mu_{\min }\right)$.

\subsubsection{Measurements of $I-V$ characteristics}

All the measurements of $I-V$ characteristics of tetracene crystals discussed in this Chapter have been performed in vacuum $\left(P<10^{-5}\right.$ mbar $)$ and in the dark, in a two-terminal configuration. We have used a Keithley 237 source-measure unit that permits to apply up to $1100 \mathrm{~V}$ across our samples and to measure currents as small as $10 \mathrm{fA}$. Measurements at lower temperature were performed in the vacuum chamber of a flow cryostat. Approximately 100 samples have been measured at room temperature. Temperature dependent measurements have been performed on most samples in which a high value for $\mu_{\min }\left(0.1 \mathrm{~cm}^{2} / \mathrm{Vs}\right.$ or better $)$ has been found and on few of the others.

The precise shape of the $I-V$ characteristics measured on different samples exhibit large deviations, whose possible origin is discussed in the next Section. Here, we focus on some important common features observed in many of the samples investigated $(\simeq 100)$. In particular, we often observe that in the lower voltage range $(V<10-100 \mathrm{~V}$, depending on the sample) the current increases with voltage is approximately quadratic [12]. At higher voltage, the current increases by many decades (typically six to eight, depending on the sample) for 


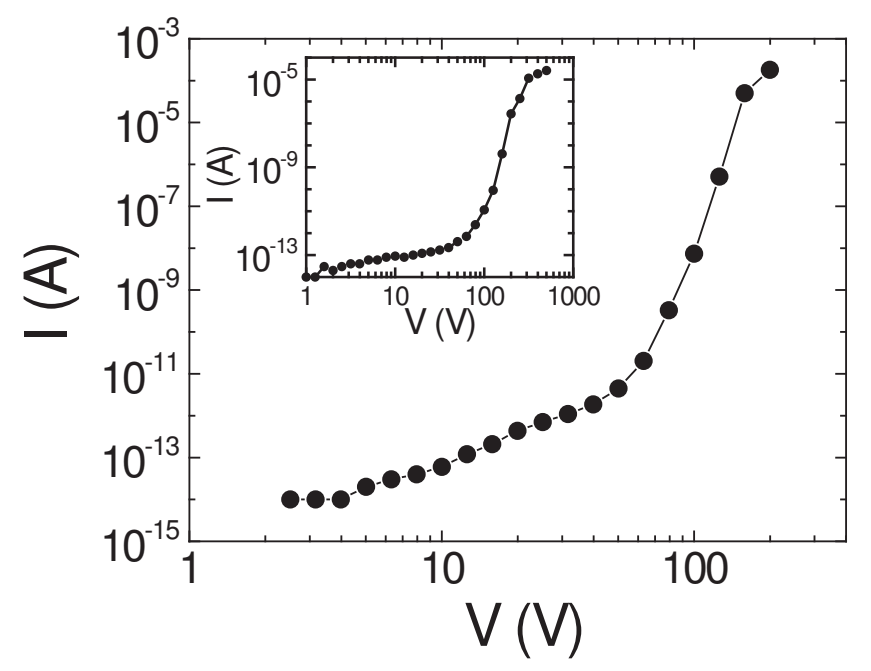

Figure 4.1: Typical result of a DC $I-V$ measurement perpendicular to the $a b$-plane of a tetracene single crystal, with a thickness $L=30 \mu \mathrm{m}$ and a mobility $\mu_{\min }=0.59$ $\mathrm{cm}^{2} /$ Vs. The inset shows a similar measurement on a different crystal $\left(L=25 \mu \mathrm{m}, \mu_{\text {min }}\right.$ $=0.014 \mathrm{~cm}^{2} / \mathrm{Vs}$ ), in which a crossing over into an approximately quadratic dependence on voltage is visible at high voltage. In both cases, a very steep current increase occurs around or just above $100 \mathrm{~V}$ that we attribute to filling of deep traps. We observed a steep increase in current in most samples studied.

a one-decade increase in voltage (Fig. 4.1). In most cases samples fail as the voltage is increased in this part of the $I-V$ curve, either because too much power is dissipated through the crystals (samples with high $\mu_{\min }$ ) or because the electrical contacts detach from the crystal (samples with low $\mu_{\min }$ ). In a few cases, however, we have observed that the rapid current increase terminates by crossing over into an approximately quadratic dependence on voltage (Fig. 4.1, inset).

The value of the current measured at the maximum applied voltage is used to calculate $\mu_{\text {min }}$ from Eq. 4.2. The calculation requires the knowledge of the crystal thickness, which, in this geometry, corresponds to the electrode separation $L$. The measurement of $L$ is done by inspecting the crystals under an optical microscope. The uncertainty of $L$ (typically 10 to $20 \%$ ) is rather large and due to the difficulty of the measurement and to the opposite crystals surfaces not being parallel to each other. In calculating $\mu_{\min }$ we have used lower estimates of $L$, in order to be sure not to over-estimate the crystal mobility.

The histogram shown in Fig. 4.2 provides an overview of the values of $\mu_{\text {min }}$ obtained from all measured samples. The spread in the calculated values of $\mu_{\min }$ is large, as a consequence of the large deviations observed in the measured $I-V$ 


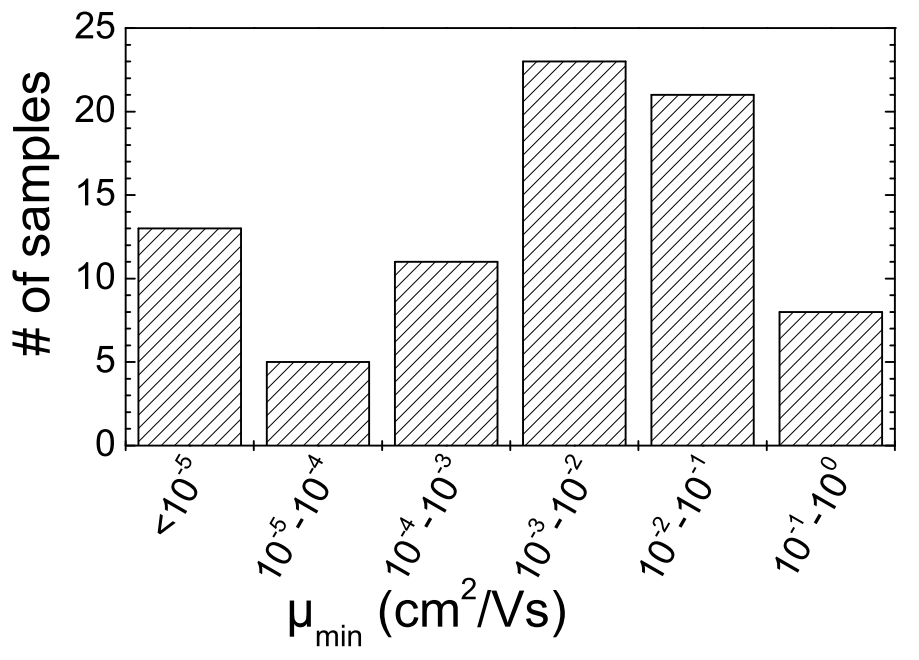

Figure 4.2: Histogram of values for $\mu_{\min }$ calculated from DC $I-V$ measurements performed on approximately 100 tetracene single crystals.

characteristics. In what follows we concentrate on those samples for which the lower limit to the mobility is $\mu_{\min }>0.1 \mathrm{~cm}^{2} / \mathrm{Vs}$ [13].

For most high-quality samples that did not fail during the room-temperature measurements we have performed $I-V$ measurements at different temperatures. We reproducibly find that $\mu_{\min }$ increases upon lowering temperature in all the samples in which $\mu_{\min }>0.1 \mathrm{~cm}^{2} / \mathrm{Vs}$ at room temperature (Fig. 4.3). The same behavior has also been observed in a couple of samples in which $\mu_{\min } \simeq 0.01$ $\mathrm{cm}^{2} / \mathrm{Vs}$, although normally samples for which $\mu_{\text {min }}<0.1 \mathrm{~cm}^{2} / \mathrm{Vs}$ at room temperature exhibit a decrease in current as the temperature is lowered. We conclude that samples in which the room-temperature mobility is sufficiently close to the value of $1 \mathrm{~cm}^{2} / \mathrm{Vs}$ exhibit the behavior expected for high-quality organic semiconductors, i.e. an increasing mobility with lowering temperature. This is the first time that this behavior is reported in simple two-terminal DC $I-V$ characteristics.

In all the high-mobility samples measured we observe that below $T \simeq 180 \mathrm{~K}$, $\mu_{\min }$ starts to decrease when the temperature is decrease further. In most samples, the change in the temperature dependence of $\mu_{\text {min }}$ is very sharp (Fig. 4.3). This suggests that the origin of this change is a structural phase transition, which is known to occur in tetracene in this temperature range.

Past studies of this transition [14] have shown that the precise transition temperature depends on details, such as the stress induced by the adhesion between a 
crystal and the substrate on which the crystal is mounted [15]. These studies have also shown that the phase transition does not occur uniformly, with two different crystalline structures coexisting in different parts of a same crystal in a large interval of temperatures below the transition. The coexistence of different crystal phases is detrimental for transport, since it introduces grain boundary junctions and regions with different bandwidth that can trap large amounts of charge carriers. This explains the observed temperature dependence of the mobility. The observation of the effect of this structural phase transition on the transport properties of tetracene provides one additional indication that the crystal quality is high and that we are probing intrinsic effects in the material.

\subsubsection{Deep traps in the bulk and at the contacts}

The interpretation of experimental data in terms of $\mu_{\min }$ is of general validity and it does not require any assumption regarding the sample characteristics. Additional information can be extracted from the measurements if one considers the behavior of the measured $I-V$ curves in more detail. Here we consider the effect of deep traps present in the bulk of the tetracene crystals and at their surface.

In general, deep traps suppress current flow by localizing charge carriers. In the space-charge limited transport regime, it can be easily shown [11] that when the applied voltage $V$ is approximately equal to

$$
V_{T F}=\frac{N_{t}^{d} e L^{2}}{\epsilon \epsilon_{0}}
$$

the charge injected by the contacts is sufficient to fill all the traps and transport occurs in the so-called trap-filled limit. At this point (i.e., with increasing $V$ from below to above $V_{T F}$ ) the measured current exhibits a large sudden increase given by:

$$
\frac{N_{t}^{d}}{N_{v}} \exp \left(\frac{E_{t}^{d}}{k_{B} T}\right)
$$

In this expression, $N_{v}$ is the number of states in the valence band which we take to be one state per molecule [11].

Essentially all samples exhibit a large, steep increase in current around a given (sample dependent) voltage (Fig. 4.1), which we interpret as due to the transition to the trap filled limit. Using Eq. 4.3 we obtain $N_{t}^{d} \simeq 5 \cdot 10^{13} \mathrm{~cm}^{-3}$. We find that different samples all give comparable values. Introducing this value for $N_{t}^{d}$ in Eq. 4.4 we then find, taking the magnitude of current increase measured on samples with the highest value of $\mu_{\min }, E_{t}^{d} \approx 700 \mathrm{meV}$. 


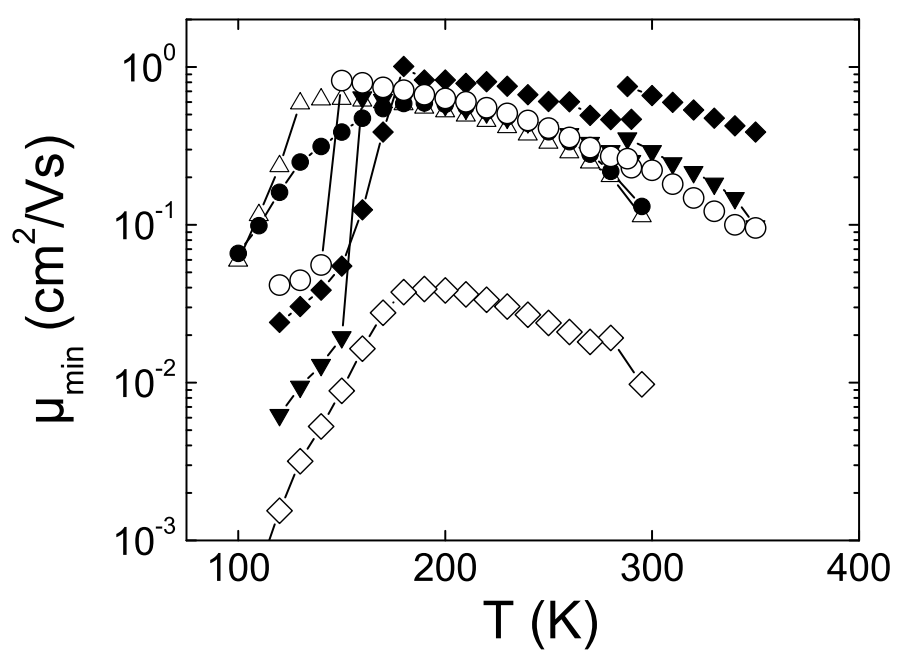

Figure 4.3: Temperature dependence of the lower limit to the mobility, $\mu_{\min }$, measured for several tetracene single crystals. Note the abrupt drop in mobility occurring at different temperatures below $\simeq 180 \mathrm{~K}$, originating from a known structural phase transition [14].

The estimate of $N_{t}^{d}$ is based on the assumption, not usually emphasized in literature, that the deep traps are uniformly distributed throughout the entire crystal bulk. In actual samples, due to the contact preparation process, it is likely that more traps are present at the crystal surface under the electrodes. A small amount of traps located close to the surface can have a large effect in suppressing current flow. This is because charges trapped at the surface can substantially affect the electrostatic profile in the bulk of the crystal, which determines the current flow in the space-charge limited current regime.

To make this point more explicit, consider a $20 \mu \mathrm{m}$ thick crystal in which no traps are present apart from traps in the first monolayer of molecules close to the surface. Suppose that, in this monolayer, one deep trap per every 1000 molecules is present. This will result in a very large current suppression, as populating these traps results in an electric field through the crystal which corresponds, in the case considered, to approximately 1000 Volts applied between the electrodes. For an $I-V$ measurement this would imply that, as the voltage across the electrodes is increased, the surface traps are initially filled and no current flows until more than 1000 Volts are applied. For comparison, for a $20 \mu \mathrm{m}$ thick crystal with no surface traps and a bulk density of traps of $5 \cdot 10^{13} \mathrm{~cm}^{-3}$ transport already occurs in the trap free limit when $200 \mathrm{~V}$ are applied across it. For this reason, 
our estimate of $N_{t}^{d}$ is a higher limit to the bulk density of deep traps.

The strong sensitivity of the $I-V$ curves to traps located at the metal/organic interface makes these traps a logical explanation for the large sample to sample variation observed in the experiments. Evidence for the relevance of contact effects is provided by the rather good sample-to-sample reproducibility observed in TOF measurements (see Section 4.4.2) as compared to DC transport measurements.

\subsection{TOF experiments on tetracene single crys- tals}

\subsubsection{Technical aspects of TOF experiments}

The Time-of-Flight (TOF) spectroscopy is based on two fundamental steps. First, by photo-excitation electron hole pairs are generated near the crystal surface. Second, in the applied electric field the charge carriers move to the electrodes and the corresponding displacement current is measured [10]. Therefore, studies on the transport behavior are free of contact effects and the technique is selective on the respective type of charge carrier by choice of the polarity of the external voltage.

For the TOF measurements the tetracene crystals are covered on both sides by a thin layer of $\mathrm{Ag}$ thermally evaporated and, afterwards, mounted on a $\mathrm{Cu}$ support acting as back-electrode as well as thermal contact for the temperature dependent studies. The $15-20 \mathrm{~nm}$ thick Ag contacts showing almost bulk conductivity but are still sufficiently transparent for photo-induced generation of charge carriers at the front electrode. As light source for the charge carrier generation, a nitrogen laser in single shot mode $(\lambda=337 \mathrm{~nm}$, pulse width 0.76 ns) is used. The suitability of the used wavelength for photo-excitation has been proven by UV-VIS absorption measurements on the crystals. In addition, from the TOF pulse shape and the absorption spectra we can conclude that charge carrier generation takes place in the topmost fraction of the crystals mainly, i.e. the depth of charge carrier generation (several $\mu \mathrm{m}$ ) can be neglected with respect to the thickness of the crystals $(\sim 100 \mu \mathrm{m})$.

The displacement current is measured as a voltage drop across a resistor connected in parallel to the crystal. The resistor is chosen in such a way that the time constant of the RC-part is much smaller than the transit time $\tau$. TOF studies in the range from room temperature up to $450 \mathrm{~K}$ were carried out in a heating stage at ambient pressure, the upper temperature limit caused by sublimation of 


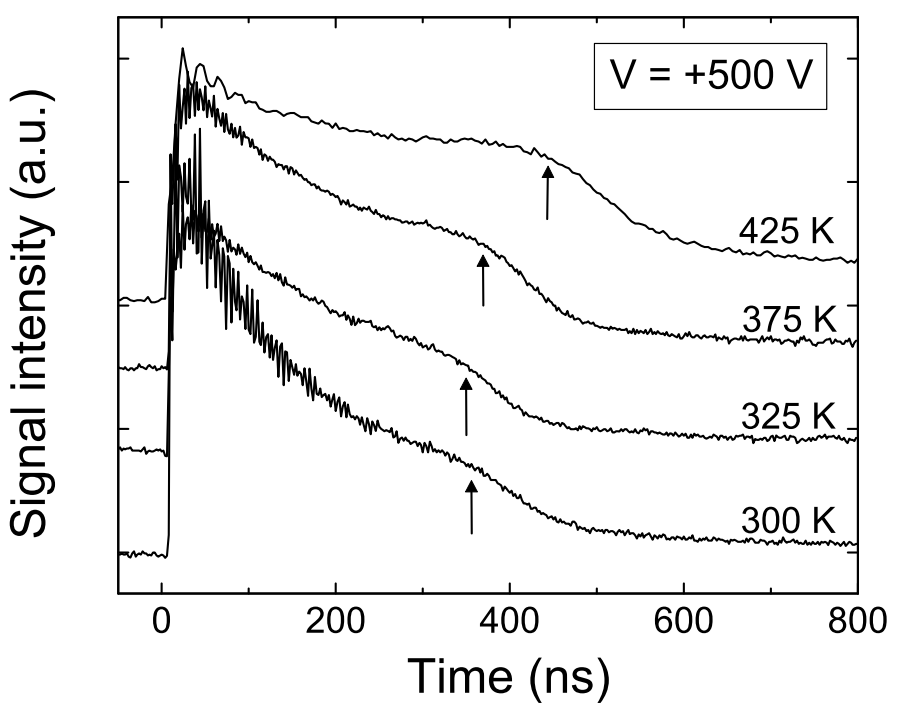

Figure 4.4: Hole TOF pulses measured at different temperatures in the range from room temperature and $150{ }^{\circ} \mathrm{C}$. The applied voltage is $+500 \mathrm{~V}$. The arrows point to the transit times.

tetracene at around $450 \mathrm{~K}[16]$.

\subsubsection{Measurement of TOF transients}

Temperature dependent TOF measurements have been performed on three different single crystals giving essentially identical results. The room-temperature mobility values obtained from the different crystals were very close to each other (ranging from $0.5 \mathrm{~cm}^{2} / \mathrm{Vs}$ to $0.8 \mathrm{~cm}^{2} / \mathrm{Vs}$ ) and their temperature dependence was qualitatively identical. This is in net contrast with the large sample-to-sample variations observed in DC transport measurements. From the outcome of the TOF experiments we conclude that the quality of the grown crystals is rather reproducible and that the sample to sample variations observed in the DC $I-V$ originates from irreproducibility in the contact preparation [17]. This is consistent with the fact that TOF experiments are not very sensitive to the contact quality whereas DC $I-V$ measurements are.

Representative TOF pulses for positive charge carriers measured at various temperatures in one of the three tetracene single crystals are shown in Fig. 4.4, from which the transit time $\tau$ can be easily extracted. In contrast to these well-defined TOF pulses measured for hole transport, only dispersive transport is observed for electrons throughout the measured temperature range. This in- 
dicates strong trapping for electrons, which is why in Section 4.3 we have used single carrier space-charge limited current theory to interpret the behavior of the measured $I-V$ curves.

Assuming a constant electric field $E$ across the crystal, the mobility of the holes is related to the transit time and to the crystal thickness $L$ by:

$$
\mu=\frac{L}{E \tau}
$$

For the values of electric field used in our studies $\tau^{-1}$ depends linearly on $E$ (see Fig. 4.5) so that $\mu$ does not depend on electric field. All the mobility values discussed in this Chapter have been estimated from this ohmic regime only.

In the absence of traps Eq. 4.5 represents the intrinsic mobility $\mu$ of the organic material, which typically varies as an inverse power of temperature, i.e. $\mu \propto T^{-n}$ ( $n \simeq 2-3$ depending on the specific organic molecules [10]). If shallow traps [18] are present, however, the measured mobility is just an "effective" mobility $\mu_{\text {eff }}$ related to the $\mu$ by [10]:

$$
\mu_{\mathrm{eff}}(T)=\frac{\mu(T)}{1+\frac{N_{t}^{s}}{N_{v}}\left[\exp \left(\frac{E_{t}^{s}}{k_{B} T}\right)-1\right]}
$$

where $N_{t}^{s} / N_{v}$ is the density ratio between shallow traps and organic molecules in the crystal and it is assumed that all the shallow traps have the same energy depth $E_{t}^{s}$ relative to the valence band. A plot of the measured $\mu_{\mathrm{eff}}$ vs. $T$ is shown in Fig. 4.6. The saturation with lowering temperature is a characteristic signature of shallow traps.

The effect of shallow traps is not only visible in the temperature dependence of the mobility, i.e. of the transit time of TOF pulses, but also in their shape. Specifically, Fig. 4.4 shows that the signal intensity increases with increasing temperature and that the pulse becomes more rectangular. Both effects are due to reduced trapping at elevated temperature, which make the sample behave more closely to the ideal trap-free condition.

We have attempted to use Eq. 4.6 to fit the measured temperature dependence of $\mu_{\mathrm{eff}}$ and to determine the values of $N_{t}^{s}$ and $E_{t}^{s}$. However, the limited temperature range which is experimentally accessible, the unknown intrinsic roomtemperature mobility of tetracene and of the value $n$ determining its temperature dependence make it impossible to determine trap density and concentration precisely. Even when we set $\mu(300 \mathrm{~K})=1 \mathrm{~cm}^{2} / \mathrm{Vs}$ and $n=2$, we find that different combinations of $N_{t}^{s}$ and $E_{t}^{s}$ produce a satisfactory fit of our data. From this analysis we can however roughly estimate that $N_{t}^{s} \approx 10^{18} \mathrm{~cm}^{-3}$ and $E_{t}^{s} \approx 100$ meV. A more precise determination of these parameters would require to extend 


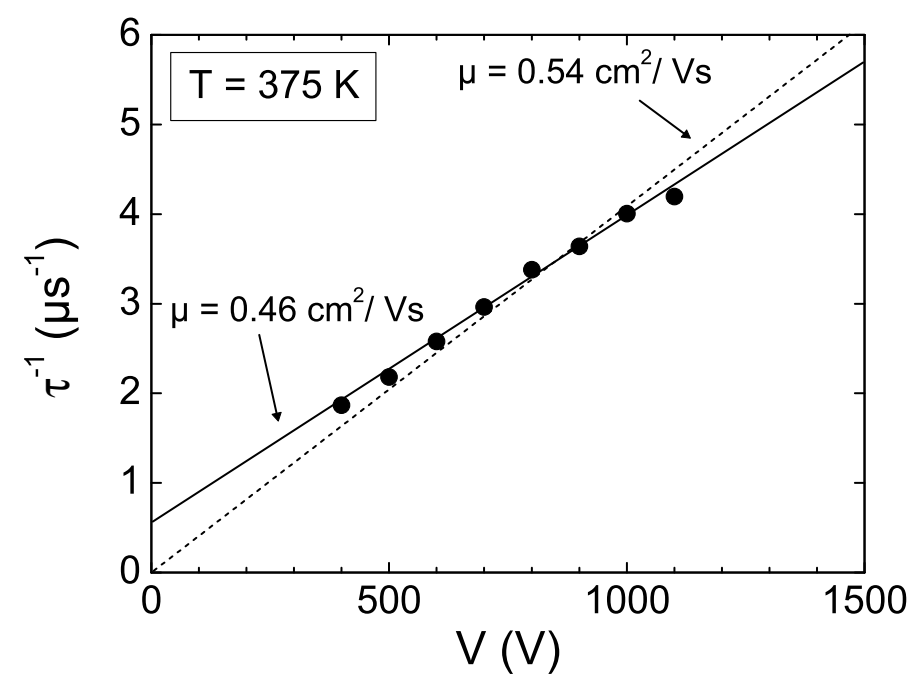

Figure 4.5: Transit time of hole TOF pulses versus applied voltage estimated at 375 K showing a linear (Ohmic) dependence. The continuous and dotted lines represents, respectively, the best linear fit and the best linear fit passing through the origin. The corresponding difference in the $\mu$ values gives a measure of the uncertainty in the extracted mobility.

the temperature dependent measurements over a wider temperature range. In practice, however, the temperature range is limited by sublimation of tetracene $(T>450 \mathrm{~K})$ on the high end and by the blurring of the TOF pulse on the low end $(T<300 \mathrm{~K})$.

\subsection{Summary}

This is the first time that systematic DC transport measurements and TOF experiments have been performed on identically grown single crystals of organic molecules and it is interesting to find overall agreement in the comparison of the results obtained with the two techniques. Specifically, from both measurement techniques we conclude that the room temperature hole mobility is close to $1 \mathrm{~cm}^{2} / \mathrm{Vs}$ and that its temperature dependence is non-monotonic [19]. For TOF measurements on tetracene, this behavior had been observed previously [20]. On the contrary, an increase in mobility with lowering temperature observed by measuring the DC $I-V$ curves in the space-charge limited transport regime had not been reported previously neither for tetracene nor, to the best of our knowledge, for any organic un-doped semiconductor. This observation, 


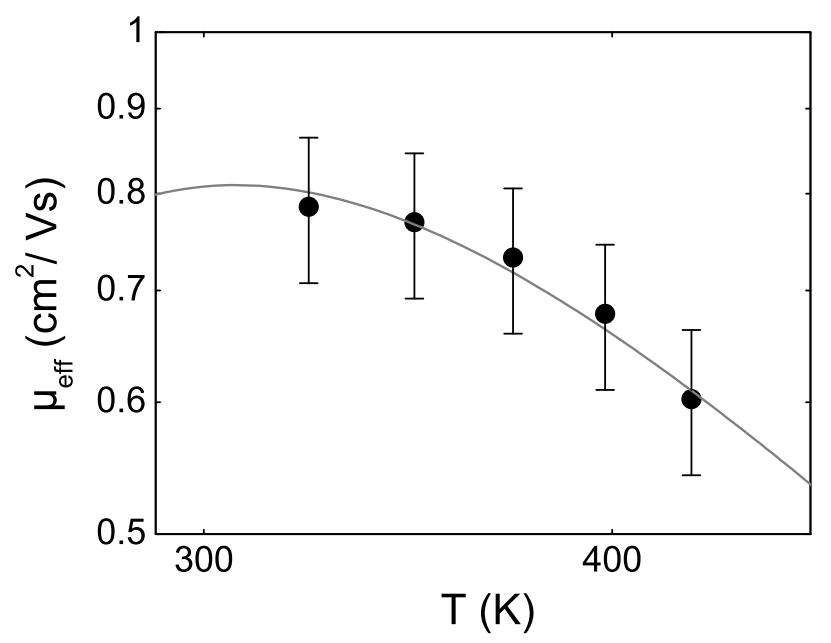

Figure 4.6: Temperature dependence of the hole mobility (full circles). The fit (straight line) is described by Eq. 4.6 using an exponent $n=2$, a mobility of 1.4 $\mathrm{cm}^{2} / \mathrm{Vs}$, a trap energy of $0.13 \mathrm{eV}$ and a density ratio between shallow traps and tetracene molecules of $5 \cdot 10^{-3}$.

together with the observed effect of the structural phase transition on the hole mobility, indicate that signatures of the intrinsic electronic properties are visible in the $I-V$ measurements, which demonstrates the high quality of the crystals.

In spite of the crystal quality, the effect of imperfections is still clearly visible. In particular, $I-V$ and TOF measurements provide complementary information about the presence of deep and shallow traps. $I-V$ measurements allow us to infer an upper limit for the bulk density of deep traps and their activation energy (see Section 4.3.3). For shallow traps the observation of a maximum in mobility in TOF measurements around room temperature indicates that the typical activation energy is $\sim 100 \mathrm{meV}$, but a more precise value as well as a reliable estimate of the density cannot be presently obtained. We believe that these shallow traps originate from local deformations of the crystals, such as those due to mechanical stress or to electrically-inactive chemical impurities as well as from chemical impurities interacting only weakly with the surrounding host molecules. Within a conventional band picture it is easy to see that these deformations would have an important effect, as they can change locally the band gap of tetracene and form spatially localized "pockets" of holes. This mechanism could account for a fairly large density of shallow traps and for a trapping energy of $\sim 100 \mathrm{meV}$ [21], which corresponds to only a few percent change in the $3 \mathrm{eV}$ tetracene gap. 
Not only the similarities but also the differences between $I-V$ and TOF measurements provide useful information. Particularly noticeable is the reproducibility of TOF measurements in contrast to the large sample to sample deviations observed in the $I-V$ characteristics. Three out of three crystals studied by means of TOF gave $\mu \simeq 0.5-0.8 \mathrm{~cm}^{2} / \mathrm{Vs}$, whereas out of approximately 100 sample measured with $I-V$ characteristics only 5 to $10 \%$ gave a mobility larger than $0.1 \mathrm{~cm}^{2} /$ Vs. The reproducibility obtained in TOF measurements indicate that different tetracene crystals grown in our set-up exhibit only minor differences in their properties and that these differences cannot account for the large spread of $I-V$ characteristics observed experimentally.

We conclude that the large sample to sample variations observed in the measurement of $I-V$ characteristics mainly originate from the quality of the electrical contacts. As mentioned above, this is critically important for $I-V$ measurements, but not for TOF measurements. Since the large values of $\mu_{\min }$ obtained in the best samples indicate that it is possible to fabricate "high-quality" contacts using silver epoxy, we infer that the effects determining the contact quality in our samples are mainly of extrinsic nature. Our estimates suggest that deep-traps present under the contacts at the crystal surface (introduced during the contact fabrication) play an important role. This is because even a very small surface density of these traps can substantially perturb the electrostatic profile in the crystal bulk that determines the current flow in the space-charge limited transport regime.

\section{References}

[1] G. H. Gelinck, T. C. T. Geuns, and D. M. de Leeuw, Appl. Phys. Lett. 77, $1487(2000)$

[2] C. D. Dimitrakopoulos, and P. R. L. Malenfant, Adv. Mater. 14, 99 (2002)

[3] I. H. Campbell, and D. L. Smith, Solid State Phys. 55,1 (2001)

[4] W. Warta, and N. Karl, Phys. Rev. B 32, 1172 (1985)

[5] N. Karl, K. -H. Kraft, J. Marktanner, M. Münch, F. Schatz, R. Stehle, and H. -M. Uhde, J. Vac. Sci. Technol. A 17, 2318 (1999)

[6] We define shallow traps as traps with an energy within a few times $k_{B} T$ from the edge of the valence band. Deep traps are further separated from the valence band.

[7] R. A. Laudise, Ch. Kloc, P. G. Simpkins, and T. Siegrist, J. Crystal Growth 187, 449 (1998) 
[8] J. Monteath Robertson, V. C. Sinclair, and J. Trotter, Acta Cryst. 14, 697 (1961)

[9] D. Holmes, S. Kumaraswamy, A. J. Matzger, and K. P. C. Vollhardt, Chem. Eur. J. 5, 3399 (1999)

[10] R. Farchioni, and G. Grosso, Organic electronic Materials (Springer-Verlag, Berlin, 2001)

[11] M. A. Lampert, and P. Mark, Current Injection in Solids(Academic Press Inc., New York, 1970)

[12] In samples with low $\mu_{\min }$ this regime may not be visible, as the current is below the sensitivity of the measuring apparatus.

[13] $I-V$ measurements performed on crystals that were regrown three or more times do not show a statistically significant difference from crystals that were regrown twice.

[14] U. Sondermann, A. Kutoglu, H. Bässler, J. Phys. Chem. 89, 1735 (1985)

[15] The presence of stress is also responsible for entire crystals breaking into small pieces upon cooling to low temperature. The temperature at which crystals beak can be very different for different crystals and it seems to depend on the cooling speed.

[16] J. D. Cox, G. Pilcher, Thermochemistry of Organic and Organometallic Compounds (Academic Press, New York, 1970)

[17] Additional evidence for contact effects is given by the asymmetry in the $I-V$ curves and polarity dependent hysteresis often observed in samples with low $\mu_{\min }$.

[18] The impact of a low density of deep traps $\left(E_{t}^{d} \gg k_{B} T\right)$ is of minor importance for TOF experiments, contrary to the case of $I-V$ measurements, since for these traps the relaxation time is much longer than the transit time and hole captured by deep traps do not give any signal.

[19] Although the general trend in the $T$ dependence of the mobility is similar in both the $I-V$ and TOF experiments, the temperature at which the mobility reaches its optimum value is different. We suggest that the cause of this difference is that, in the case of $I-V$ measurements, a much larger amount of charge is being injected, so that more (shallow) traps are filled.

[20] J. Berrehar, P. Dellanoy, and M. Schott, Phys. Stat. Sol. (b) 77, K119 (1976)

[21] E. A. Silinsh, and V. Cápek, Organic Molecular Crystals: Interaction, Localization, and Transport Phenomena (AIP Press, Woodbury, 1994) 


\title{
Chapter 5
}

\section{Influence of surface traps on space-charge limited current}

\begin{abstract}
We analyze the effect of surface traps on unipolar space-charge limited current and find that they have a profound influence on the $I-V$ curves. By performing calculations that account for the presence of these traps, we can reproduce experimental observations not captured by the conventional theory that only considers the presence of traps in the bulk of the material. Through the use of realistic material parameters, we show that the effects discussed have clear experimental relevance.
\end{abstract}

This Chapter has been published as R. W. I. de Boer, and A. F. Morpurgo, Phys. Rev. B 72, 073207 (2005) 


\subsection{Introduction}

Space-charge limited transport occurs in un-doped, wide-gap semiconductors in which the density of charge carriers at equilibrium is vanishingly small. In these materials, the current is carried by charge injected from the contacts, whose density is determined (limited) by electrostatics. Space-Charge Limited Current (SCLC) is relevant for the operation of electronic devices (e.g., organic light emitting diodes) and is routinely used in the characterization of novel semiconductors to estimate parameters such as the mobility of charge carriers, the density of trap states, and their energy depth $[1,2,3,4,5]$.

The description of SCLC relies on a simple phenomenological theory first developed in the fifties and extended later to include model specific details $[6,7]$. These extensions have led to predictions of SCLC $I-V$ characteristics that resemble rather closely what is actually measured. In practice, however, the comparison between SCLC measurements and theory is not very satisfactory in many cases, especially for experiments performed on high-quality materials with a low density of defects. Firstly, an independent validation of specific assumptions adopted in the analysis of SCLC $I-V$ curves is almost always impossible, which causes ambiguities in the interpretation of the experiments. Even when the theoretically predicted $I-V$ characteristics exhibit a behavior close to that observed experimentally, a sufficiently detailed analysis often reveals inconsistencies [8]. Secondly, SCLC $I-V$ curves measured on nominally identical samples often exhibit significant differences [3], which makes it inappropriate to compare experimental data with theoretical predictions that are critically sensitive to the assumptions on which models rely. This current situation suggests that some important aspects of the physics of space-charge limited transport are being overlooked.

In this context, and motivated by recent experimental work on high-quality organic single crystals, we investigate the effect of deep traps at the surface of the semiconducting material, underneath the electrical contacts used to inject charge carriers. We show that these surface traps are an essential ingredient for the proper understanding of SCLC $I-V$ curves, which has been neglected until now. In particular, surface traps cause a large change in the electrostatic profile throughout the bulk of the material, profoundly affecting the behavior of SCLC. Calculations accounting for the presence of surface traps enable us to reproduce experimental observations that are not captured by the conventional theory, such as orders-of-magnitude asymmetries in the $I-V$ curves. These calculations, which do not require any detailed, model-specific assumption, further illustrate how the combined effect of surface and bulk traps also results in features in the 
$I-V$ curves that have been so far attributed to different physical mechanisms. In this way, our work provides the correct framework for the interpretation of SCLC measurements and may explain inconsistencies often found in the analysis of past experimental results.

\subsection{Modifications to the conventional SCLC the- ory}

To understand how the effect of surface traps is taken into account in our calculations, we first briefly review the main aspects of the conventional theory of unipolar SCLC [6]. The theory relies on the simultaneous solution of the Poisson and the continuity equation,

$$
\frac{d E(x)}{d x}=\frac{e n_{s}(x)}{\epsilon}
$$

and

$$
J(x)=e n_{f}(x) \mu E(x)=\text { constant }
$$

that self-consistently relate the local electric field $E(x)$ and the current density $J(x)$ ( $x$ is the distance from the injecting electrode). Here $n_{s}(x)$ is the total space charge at position $x$ and $n_{f}(x)$ is the part of space charge that is free to move, which is smaller than $n_{s}(x)$ if deep traps are present. Given the bulk density of traps $N_{t}$ and their energy depth $E_{t}$, a relation between $n_{f}(x)$ and $n_{s}(x)$ can be found via the Fermi-Dirac distribution [9]. This enables the solution of the equations above that gives the relation between $J(x)$ and $E(x)$, i.e., the $I-V$ characteristics of the material.

The bulk density of deep traps $N_{t}$ is usually assumed to be uniform throughout the material. In real materials, however, many more traps per unit volume are likely to be present close the sample surface than in the bulk. These surface traps can have different physical origins. In inorganic covalently bonded materials, for instance, they may be due to dangling bonds resulting in the presence of surface states whose energy is deep inside the semiconducting gap. In organic systems, such as van der Waals bonded molecular single crystals, traps can originate from molecules at the surface that are damaged during the contact fabrication process. For pure semiconducting materials in which the bulk density of traps is low, the total number of traps at the sample surface can be comparable or even larger than the total amount of bulk traps even for rather thick samples. The distinction between surface and bulk traps is conceptually very important. 


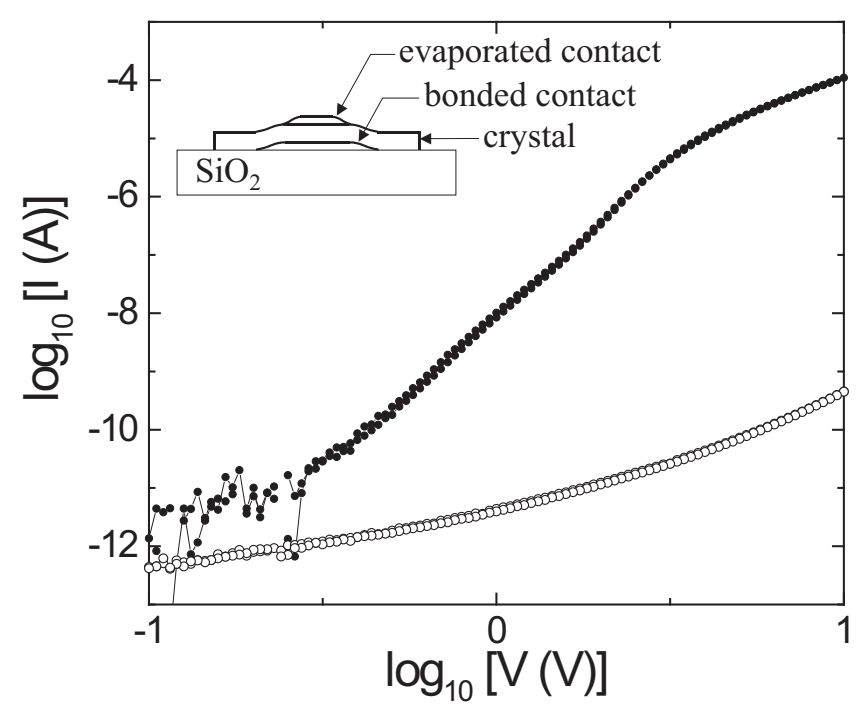

Figure 5.1: $I-V$ characteristics of a 1- $\mu$ m-thick tetracene single crystal, measured for opposite polarities of the bias voltage. The full symbols correspond to the current measured when holes are injected from the bottom (electrostatically bonded) contact (i.e., the bottom contact is at a positive bias $V$ with respect to the top contact); the open symbols correspond to the case of holes injected from the top, evaporated contact (i.e., the top contact is at a positive bias $V$ with respect to the bottom contact). The device configuration is shown in the inset.

In particular, if SCLC transport is dominated by the presence of surface traps, SCLC $I-V$ curves measured on nominally identical bulk samples (e.g., identically grown single crystals) can exhibit a large spread in their behavior, simply because the quality of the sample surface is different. This is indeed what is observed experimentally [3].

As we now proceed to show, the main effect of charge trapped at the surface is to modify the electrostatic profile throughout the entire bulk of the sample. This effect has not been appreciated and analyzed theoretically before. In spite of its conceptual relevance, the modification of the conventional SCLC theory needed to take into account the electrostatic effects due to surface traps is minimal. It is sufficient to allow $N_{t}$ to depend on position, so that its value in the region close to the contacts is much larger than the bulk value. The simplicity of this approach is important, because it permits to describe quantitatively the effect of surface traps without introducing any detailed -and experimentally not verifiablemicroscopic assumption. This makes our conclusions robust and independent of specific aspects of a specific model.

The analysis of the effect of surface traps is carried out by solving numerically 
Eq. 5.1 and 5.2 with an $x$-dependent $N_{t}$, from which we directly obtain the $I-V$ characteristics. The most interesting case is that in which the density of surface traps present under one of the contacts is considerably larger than that present under the other contact. This is experimentally relevant because in general bottom and top contacts (see inset Fig. 5.1) are prepared in different ways, which results in different surface trap densities. Also for contacts prepared in a nominally identical way, the surface density of traps at the two contacts can differ considerably because the defects introduced at the surface depend on unknown parameters that are not under experimental control $[3,10]$. We compare the simplest possible situations in which surface traps are present only under one of the two contacts (bulk traps are also present): the case of traps present at both contacts with different densities can be analyzed in an identical way and does not add new physics.

\subsection{Manifestations of surface traps in calculated $I-V$ curves}

\subsubsection{Asymmetric $I-V$ characteristics}

Our considerations are valid for different classes of materials. Nevertheless, here we will mainly have in mind the case of organic molecular single crystals (such as tetracene, rubrene, the metal phthalocyanines, etc.), to which we have recently devoted considerable experimental effort. This enables us to insert in our calculations realistic values of the parameters and to prove that the effect of surface traps is relevant in actual experiments.

An illustrative example of SCLC through tetracene (a hole conductor) crystals is shown in Fig. 5.1. The data are measured on an approximately $1-\mu$ m-thick single crystal contacted with two gold electrodes prepared in different ways. One of the contacts is fabricated by placing the thin crystal onto a metal film deposited on a substrate to which the thin, flexible crystal adheres spontaneously. This procedure is known to result in high-quality electrical contacts between the metal and the crystal $[11,12,13]$. The other contact is prepared by electron-beam evaporation of gold onto the crystal, which is known to cause "damage" to the crystal due to the exposure of the crystal surface to X-Ray and high-energy electrons generated by the electron-beam, during the evaporation process. The most striking feature of the SCLC $I-V$ curves is the large (five to six orders of magnitude) asymmetry: the measured current depends very strongly on the contact used to inject holes into the material. Order-of-magnitude asymmetries 
are regularly found for different contact fabrication techniques.

Fig. 5.2 shows the $I-V$ characteristics calculated for a sample with bulk traps and surface traps under only one of the two contacts. The separation between the contacts is taken to be $1 \mu \mathrm{m}$, corresponding to the case of the device whose data are shown in Fig. 5.1. The bulk density of traps is taken to be $1 \cdot 10^{14} \mathrm{~cm}^{-3}$. This is a conservative, realistic estimate [3] and lower values [5] would result in a more pronounced effect of the surface traps. The surface density of traps is set to correspond approximately to one trap per every 1000 molecules in the first few molecular layers of the crystal, which we model as a region of approximately $10 \mathrm{~nm}$ at the crystal surface, containing a density of traps of $\sim 3 \cdot 10^{18} \mathrm{~cm}^{-3}$. For both bulk and surface traps, the energy depth is taken to be same (to avoid the insertion of unnecessary additional parameters) and equal to $0.7 \mathrm{eV}$, with the precise value of this parameter not being critical for our conclusions. Other parameters are required by the model, but do not have any considerable influence on the results. Apart from the hole mobility, which only contributes as an overall scale factor (we take $\mu=1 \mathrm{~cm}^{2} / \mathrm{Vs}$ ), there are density of dopants and their energy depth [14]. These parameters, which mainly affect the magnitude of the current in the linear regime, are treated identically to what is done in the conventional SCLC theory $[6,7]$.

The calculations show that in the range between 1 and $100 \mathrm{~V}$ the current depends very strongly on the voltage polarity. This reproduces the huge asymmetry in the $I-V$ characteristics observed experimentally. In agreement with the experiments, the current is large when the polarity is such that holes are injected from the contact free of surface traps. This result, which is robust and does not critically depend on the values of the parameters chosen above, clearly demonstrates the relevance of surface traps.

Microscopically, the asymmetry originates from a large difference in the electrostatic profile in the bulk of the samples, for the two bias polarities. This is illustrated in Fig. 5.3, where the density of charge is plotted as a function of position for the two polarities, with $10 \mathrm{~V}$ applied bias. When traps are located at the injecting contact, the bias is not sufficient to reach complete filling of the surface traps, so that no charge is injected in the bulk. The current is then carried only by thermally activated carriers and its magnitude is therefore small. On the contrary, the same bias is largely sufficient to fill the same amount of traps at the extracting contact. In this case the injected charge is present throughout the bulk of the sample, enabling a large current flow. We conclude that surface traps at the injecting contact suppress charge injection and the current flow much more drastically than trap at the extracting contact. As we will discuss at the end, this behavior can be easily understood qualitatively. 


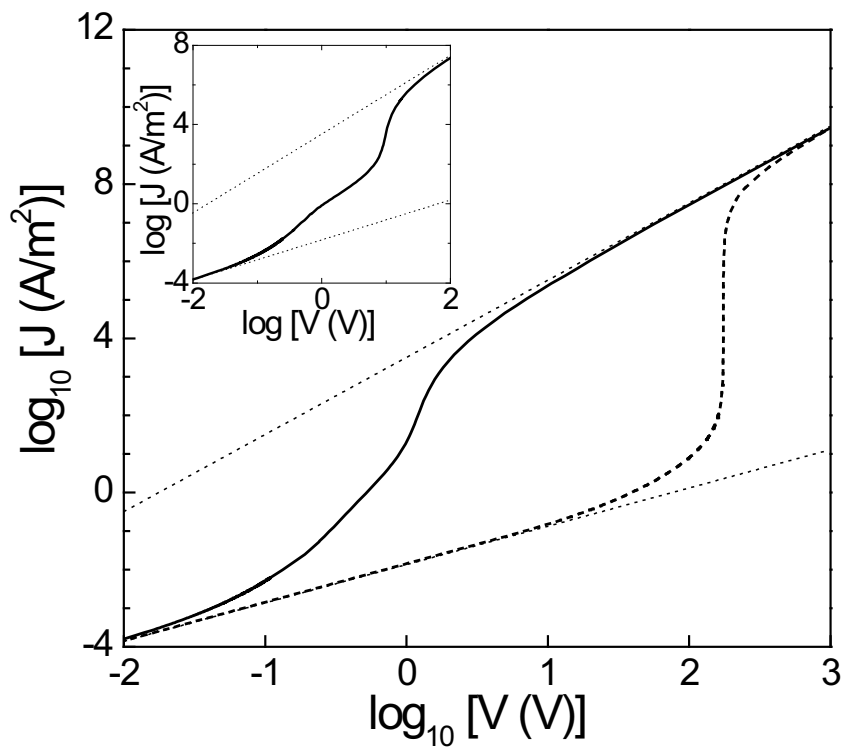

Figure 5.2: Calculated SCLC $I-V$ curve for two different polarities of the applied bias. The continuous (dashed) line represents the current flowing when holes are injected from the contact without (with) surface traps. Note the large asymmetry, which reproduces the experimentally observed behavior (see Fig. 5.1). The inset shows that, when the surface traps are located at the extracting contact, the amount of surface and bulk traps can be chosen so that the transition to the trap-filled limit exhibits multiple steps.

\subsubsection{Additional manifestations of surface traps}

Fig. 5.2 also shows that the shape of the $I-V$ curve differs, depending on the contact used to inject the carriers. If the carriers are injected from the contacts where the surface traps are, the transition is very sharp and similar to that predicted by the conventional theory when only bulk traps at a discrete energy value are present. However, if the carriers are injected from the trap-free contact, the transition from the linear to the trap-filled regime (where $I \propto V^{2}$ ) is smooth and power-law like, with an exponent larger than 2 . In this case, the precise shape of the transition is governed by the ratio between the density of surface traps at the extracting contact and the density of bulk traps. This is illustrated in the inset of Fig. 5.2, where this ratio has been changed to produce two apparent transitions in the $I-V$ curve. We note that in the analysis of experimental SCLC curves based on the conventional theory of SCLC, similar features are attributed to a distribution of energies of the bulk traps. Specifically, a power-law like $\left(I \propto V^{n}\right.$ with $n>2$ ) transition is invariably attributed to a continuous distribution of 


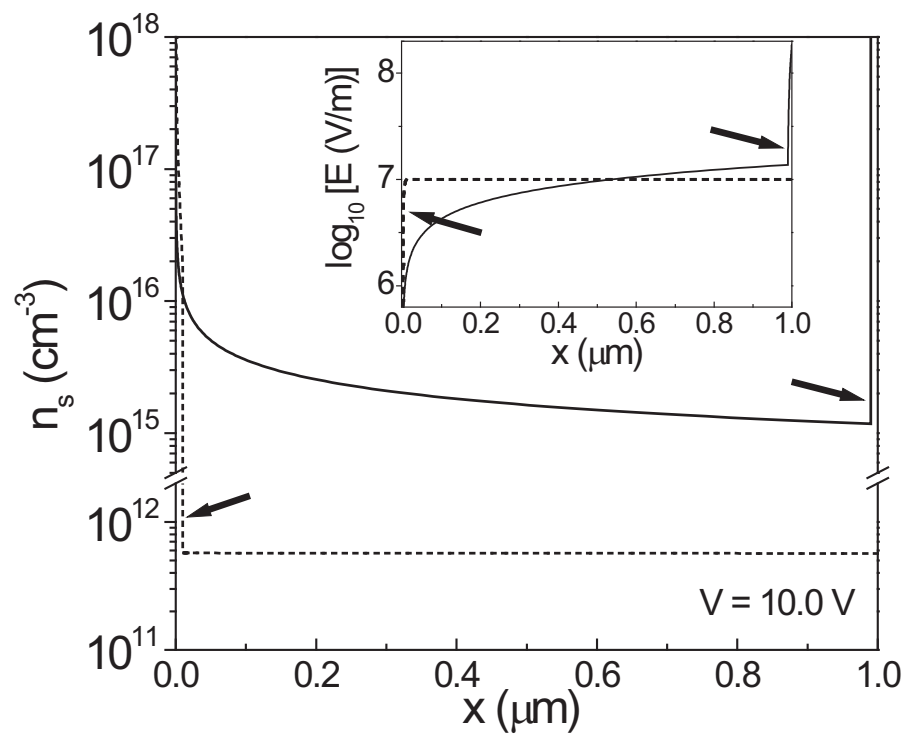

Figure 5.3: Profile of the total (trapped plus free) density of charge in the sample whose $I-V$ curves are shown in Fig. 5.1, for a $10 \mathrm{~V}$ applied bias. The injecting and extracting contacts are located at $x=0$ and $x=1 \mu \mathrm{m}$, respectively. The continuous (dashed) line corresponds to the case where the surface traps (pointed to by the arrows) are at the extracting (injecting) contact. A large density of charge is injected into the bulk when the surface traps are located at the extracting contact, but not when the traps are located at the injecting contact. In the inset, the electric field profile is shown in the two cases.

trap energies, whereas multiple discrete traps are invoked to account for multiple transitions. Our results show that such an interpretation is not unique and may explain inconsistencies found in the past [8].

One more experimentally relevant finding regards the value of the voltage $V_{T F}$ at which the transition to the trap filled limit occurs. In the conventional theory $V_{T F} \propto N_{t} L^{2}$, which is often used to estimate the bulk density of deep traps. Fig. 5.4 shows the behavior of SCLC curves when surface traps are present at the injecting contact. In this case, the qualitative shape of the SCLC curves is identical to that obtained with the conventional theory. However, the value of $V_{T F}$ is different, and is found to scale linearly with $L$ (see inset). Thus, the experimental observation of such a linear scaling provides a direct way to demonstrate the relevance of surface traps. 


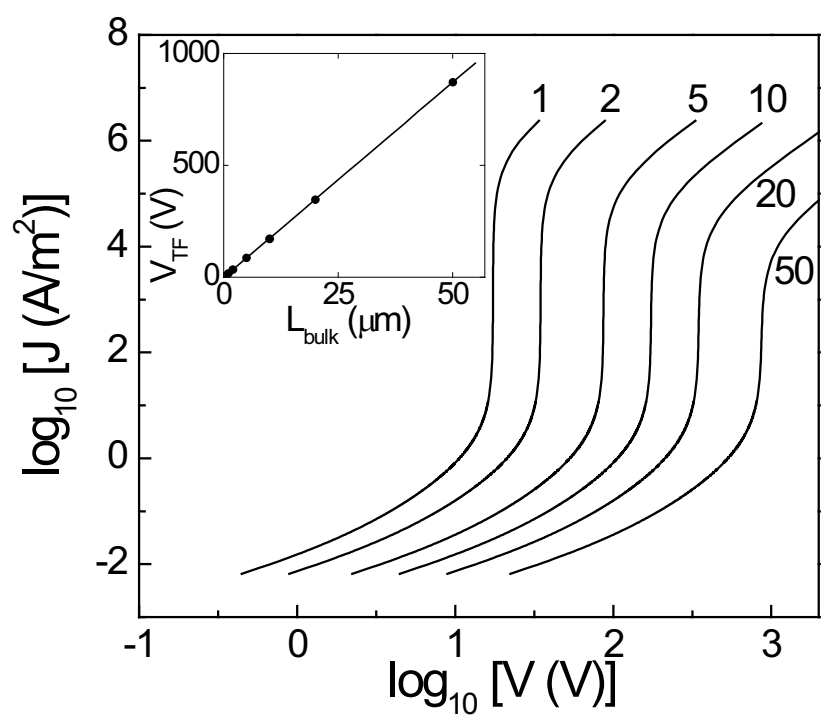

Figure 5.4: SCLC $I-V$ curves with surface traps at the injecting contact, for different contact separations $L$, ranging from 1 to $50 \mu \mathrm{m}$ (as indicated in the figure). If the total number of surface traps is larger than the total number of bulk traps, the shape of the $I-V$ curve is essentially identical to that predicted by the conventional SCLC theory. However, $V_{T F}$ scales linearly with $L$, as shown in the inset.

\subsubsection{Perturbed electrostatic profile}

The results discussed above can be understood in terms of the electrostatics of the system. In very simple terms, in a SCLC experiment a device can be thought of as a capacitor that is charged by the applied voltage. The spatial distribution of traps determines the capacitance of the device. Since in a parallel plate geometry the capacitance is inversely proportional to the distance between the charges, traps at the injecting contact have a low capacitance (the distance between the charges corresponds to the total thickness of the crystal $L$ ), so that the application of a large voltage bias is needed to fill them. Since essentially no charges are injected in the bulk until a sufficiently large voltage is applied to fill all the surface traps, this results in a strong perturbation of the electrostatics throughout the bulk of the device, which is why surface traps at the injecting contact have such a large influence on the $I-V$ curve. On the contrary, surface traps close to the extracting contact have a large capacitance, so that their filling occurs already at a very small voltage bias. On the voltage scale used in the measurements (and for sufficiently thick crystals) the perturbation to the electrostatics is only minor and does not prevent the injection of charges into the bulk. This difference directly accounts for the asymmetry of the $I-V$ curves. It also accounts for the behavior 
of $V_{T F}$ (see Fig. 5.4 inset), since the capacitance of traps located at the injecting contact scales linearly with $L$.

\subsection{Conclusion}

In conclusion, we have shown that the influence of surface traps is an essential ingredient for the proper understanding of SCLC experiments. The crucial point is that in high-purity samples, the total amount of surface traps can dominate over the total amount of bulk traps, even for sizable contact separations. Under these conditions, charge trapped at the surface strongly modifies the electrostatic profile inside the bulk, which in turn determines the amplitude of the measured current. Although SCLC measurements have been performed for over 50 years ago and their theoretical description is now textbook material, the large effect of surface traps on the electrostatics had never been recognized earlier.

\section{References}

[1] A. J. Campbell, D. D. C. Bradley, and D. G. Lidzey, J. Appl. Phys. 82, 6326 (1997)

[2] V. Podzorov, S. E. Sysoev, E. Loginova, V. M. Pudalov, and M. E. Gershenson, Appl. Phys. Lett. 83, 3504 (2003)

[3] R. W. I. de Boer, M. Jochemsen, T. M. klapwijk, A. F. Morpurgo, J. Niemax, A. K. Tripathi, and J. Pflaum, J. Appl. Phys. 95, 1196 (2004)

[4] D. V. Lang, X. Chi, T. Siegrist, A. M. Sergent, and A. P. Ramirez, Phys. Rev. Lett. 93, 076601 (2004)

[5] O. D. Jurchescu, J. Baas, and T. T. M. Palstra, Appl. Phys. Lett. 84, 3061 (2004)

[6] M. A. Lampert, and P. Mark, Current Injection in Solids (Academic Press, New York, 1970)

[7] K. C. Kao, Dielectric Phenomena in Solids (Elsevier Academic Press, San Diego, 2004)

[8] M. Pope, and C. E. Swenberg, Electronic Processes in Organic Crystals and Polymers, 2nd ed. (Oxford University Press, New York, 1999)

[9] The space charge is the sum of the free charge and the trapped charge. The trapped charge $n_{t}$ can be expressed in terms of free charge with help of the Fermi-Dirac distribution: $n_{t}=\frac{N_{t}}{1+\left(N_{v} / n_{f}\right) \exp \left(E_{t} / k_{B} T\right)}$, where $N_{v}$ is the 
density of states in the valence band, $k_{B}$ is the Boltzmann constant, $T$ the temperature and the other parameters are defined in the text.

[10] J. Reynaert, K. Poot, V. Arkhipov, G. Borghs, and P. Heremans, J. Appl. Phys. 97, 063711 (2005)

[11] J. Zaumseil, T. Someya, Z. N. Bao, Y. L. Loo, R. Cirelli, and J. A. Rogers, Appl. Phys. Lett. 82, 793 (2003)

[12] R. W. I. de Boer, T. M. Klapwijk, and A. F. Morpurgo, Appl. Phys. Lett. 83, $4345(2003)$

[13] R. W. I. de Boer, M. E. Gershenson, A. F. Morpurgo, and V. Podzorov, Phys. Stat. Sol. 201, 1302 (2004)

[14] In the regime that we are analyzing, the density of dopants is much smaller than the density of traps and their energy depth is shallower than for trap states. The conclusions of this work do not depend on the precise values. The density of states in the valence band is also needed in our calculations and it is taken to be one state per molecule $\left(3.3 \cdot 10^{21} \mathrm{~cm}^{-3}\right)$, as usually done in literature. 
Chapter 6

\section{Field-effect transistors on tetracene single crystals}

We report on the fabrication and electrical characterization of field-effect transistors at the surface of tetracene single crystals. We find that the mobility of these transistors reaches the room-temperature value of $0.4 \mathrm{~cm}^{2} / \mathrm{Vs}$. The non-monotonous temperature dependence of the mobility, its weak gate voltage dependence, as well as the sharpness of the subthreshold slope confirm the high quality of single-crystal devices. This is due to the fabrication process that does not substantially affect the crystal quality.

This Chapter has been published as R. W. I. de Boer, T. M. Klapwijk, and A. F. Morpurgo, Appl. Phys. Lett. 83, 4345 (2003) 


\subsection{Introduction}

Common strategies for the fabrication of organic field-effect transistors (FETs) are based on thin-film technology $[1,2]$. This choice is motivated by the existing deposition techniques for organic thin films that facilitate device fabrication. At the same time, thin films usually contain a considerable amount of structural imperfections, which affect negatively the transistor performance [3].

For small organic molecules, crystalline films can be used to reduce the amount of structural defects. It has been found, however, that also the performance of transistors based on these films are affected by structural imperfections, even when only one crystalline grain is present between source and drain [4]. This is due to disorder present in the first few molecular layers, in contact with the substrate, which constitute the device active region [5]. For this reason, improving the quality of organic thin-film transistors (TFTs) requires highly ordered molecular films, in which the order extend up to the interface with the substrate.

An alternative route to the production of high-quality organic FETs is to fabricate devices on the surface of a free-standing single crystal of organic molecules. If a fabrication process that preserves the quality of the crystals $[6,7]$ can be developed, the resulting single-crystal FETs should perform better than their TFT counterpart. Whereas considerable amount of work is currently aiming at improving the quality of organic TFTs $[2,4,5,8,9]$, the investigation of single-crystal organic FETs has received only limited attention [10, 11, 12, 13, 14].

In this Chapter we discuss the fabrication and electrical characterization of field-effect transistors at the surface of tetracene single crystals. The fabrication process is based on adhesion of pre-grown, free-standing crystals to a thermally oxidized Si wafer on which source and drain electrodes are deposited in advance. As we will show, this process preserves the quality of the starting crystals. From the electrical evaluation of a large number of devices we find that the mobility of the charge carriers (holes) in our single-crystal FETs is reproducibly high, reaching $0.4 \mathrm{~cm}^{2} / \mathrm{Vs}$ in the best device. In addition, the observed temperature and gate voltage dependence of the mobility as well as the subthreshold slope indicate that the performance of single-crystal devices compare well to the best existing organic thin film transistors [3].

\subsection{Electrostatic adhesion of thin crystals}

The FET fabrication involves two main steps: the growth of single crystals and the preparation of a substrate on which the crystal is subsequently placed [15]. Tetracene single crystals are grown by means of physical vapor deposition in 


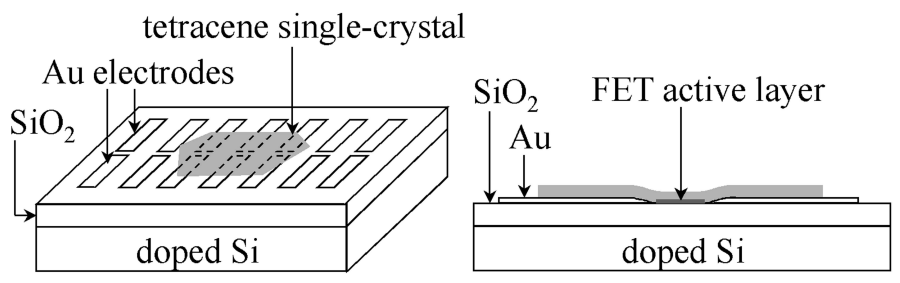

(a)

(b)

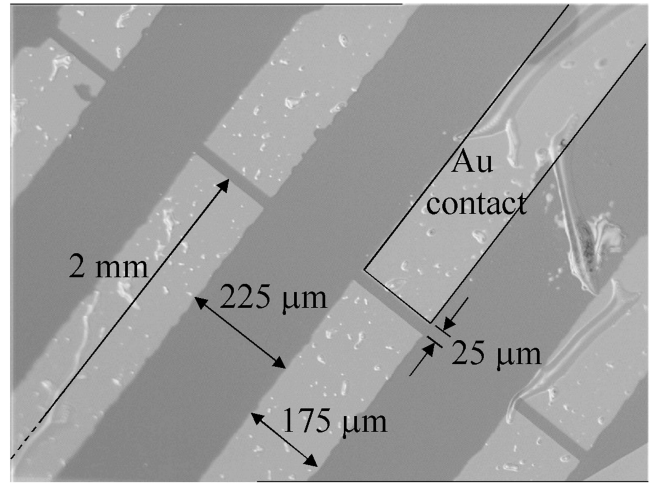

(c)

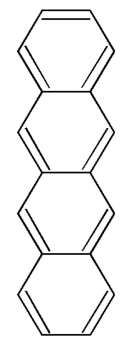

(d)

Figure 6.1: Schematic representation (a) and side view (b) of a tetracene single-crystal FET. (c) Optical microscope image of a tetracene single-crystal FET. In this device, the semi-transparent tetracene single crystal extends over several pairs of electrodes, which are clearly visible under it. In most cases smaller crystals have been used which extend over only one or two pair of contacts. These different configurations allow us to study transistors with different $W / L$ ratios on the same crystal. (d) Molecular structure of the tetracene molecule.

a temperature gradient in the presence of a stream of Argon gas. The set-up used for the crystal growth is similar to that described in Ref. [16]. The source material is $98 \%$ pure tetracene purchased from Sigma-Aldrich. Crystals grown from as-purchased tetracene are used as source material for a subsequent regrowth process, which results in crystals of increased chemical purity. Tetracene crystals grown using physical vapor deposition are platelets. For the devices described in this Chapter we select thin $(\sim 1 \mu \mathrm{m}$ thick $)$ single crystals obtained by stopping the second re-growth process at an early stage.

The substrates used for the FET fabrication are highly doped (n-type or ptype) Si wafers, covered with a layer of $200 \mathrm{~nm}$ thermally grown $\mathrm{SiO}_{2}$. The conducting $\mathrm{Si}$ wafer serves as gate electrode, with the $\mathrm{SiO}_{2}$ layer acting as gate insulator. Gold contacts are deposited on top of the $\mathrm{SiO}_{2}$ by means of e-beam evaporation through a shadow mask (see Fig. 6.1 for the precise geometry and 
dimensions). The dimensions of the $\mathrm{Au}$ contacts as well as the tetracene crystal size determine the transistor channel length $L$ and channel width $W$. This allows us to study transistors with different $W / L$ ratios. Prior to placing the tetracene single crystals on top of the substrate, the $\mathrm{SiO}_{2}$ surface is cleaned by Reactive Ion Etching (RIE) in an oxygen plasma. We found that this cleaning step is crucial for reproducible FET behavior [17].

Freshly grown tetracene crystals placed on RIE-cleaned $\mathrm{SiO}_{2}$ strongly adhere to the substrate. Adhesion only occurs for very thin crystals $(\sim 1 \mu \mathrm{m})$ that are sufficiently flexible and it is probably due to electrostatic forces. The crystals placed onto substrates are then inspected under an optical microscope using cross-polarizers. This allows us to select single-crystalline samples without visible defects for in depth electrical characterization [18]. The top view of a device fabricated following this procedure is shown in Fig. 6.1c.

\subsection{Characterization of FET performance}

We have characterized more than ten single-crystal FETs exhibiting similar overall behavior. Electrical characterization is performed in the vacuum chamber of a flow cryostat at a pressure of $10^{-7}$ mbar, using a HP4156A Semiconductor Parameter Analyzer. The measurements shown in this Chapter have been performed in a two-terminal configuration.

Fig. 6.2 shows the outcome of the measurements for one of the transistors with highest mobility. The current increases with increasing negative gate voltage. This indicates field-effect induced hole conduction, which is the expected behavior for tetracene. The field-effect mobility is evaluated in the linear regime of operation, where $I_{D}$ is proportional to $V_{D}$ :

$$
I_{D}=\frac{W}{L} \mu C_{i}\left(V_{G}-V_{T}\right) V_{D}
$$

Here $C_{i}$ is the capacitance per unit area of the $\mathrm{SiO}_{2}$ layer and $V_{T}$ is the threshold voltage. From Eq. 6.1 we obtain the mobility by calculating the derivative of $I_{D}$ with respect to both $V_{D}$ and $V_{G}$ and neglecting the dependence of $\mu$ on $V_{D}$ and $V_{G}$.

For all the FETs investigated we found room-temperature values of the mobility larger than $0.01 \mathrm{~cm}^{2} / \mathrm{Vs}$. In many cases $\mu>0.1 \mathrm{~cm}^{2} / \mathrm{Vs}$ and the maximum mobility achieved so far is $\mu=0.4 \mathrm{~cm}^{2} / \mathrm{Vs}$ (Fig. 6.2). This value is better than the highest mobility recently reported in tetracene TFTs and indicates the high quality of our single-crystal FETs [19]. 


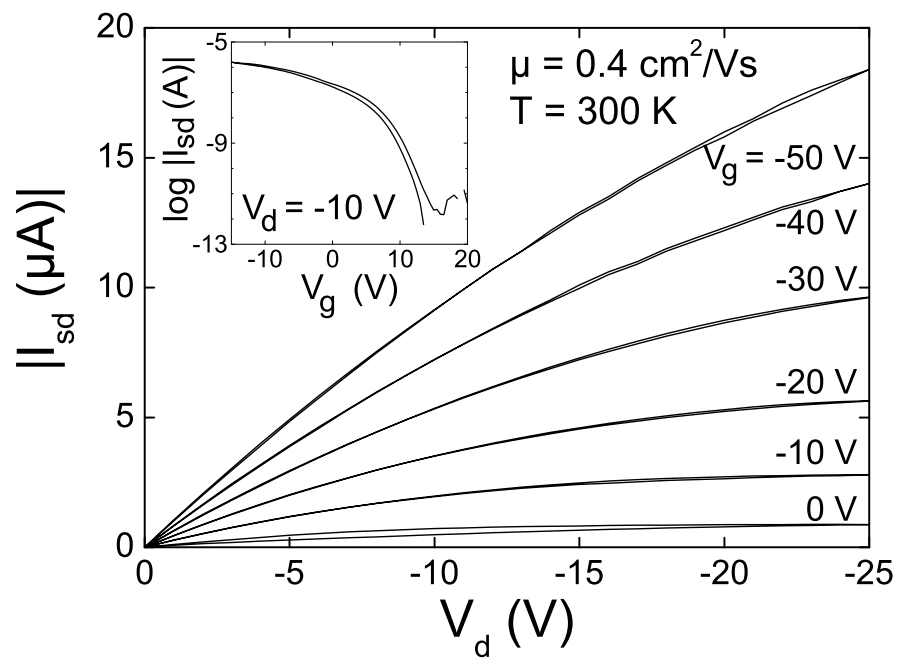

Figure 6.2: Source-drain current $I_{D}$ versus drain voltage $V_{D}$ measured at different values of $V_{G}$. The inset shows the dependence of $\log \left(I_{D}\right)$ on $V_{G}$ at fixed $V_{D}$, for a different device, which has a mobility $\mu=0.05 \mathrm{~cm}^{2} / \mathrm{Vs}$ and a threshold voltage $V_{T} \simeq 0.3 \mathrm{~V}$. From this plot we calculate the subthreshold slope to be $1.6 \mathrm{~V} /$ decade.

The threshold voltage (defined by equation 1 ) is positive in all our devices. It ranges from $0 \mathrm{~V}$ to $30 \mathrm{~V}$ and is typically $V_{T} \simeq 10 \mathrm{~V}[20]$. We do not normally observe a positive threshold voltage in FETs fabricated without the RIE cleaning, nor do we observe linear conduction through single crystals contacted with evaporated gold contacts. These observations suggest that the current flowing at zero gate voltage is not due to conduction through the crystal bulk (i.e. crystal doping) but rather to charge accumulated at the surface [21]. We believe that the charge accumulation is induced by the electrostatic adhesion of tetracene crystals to the RIE cleaned $\mathrm{SiO}_{2}$ surface.

As an additional characterization of the single-crystal FETs, the inset of Fig. 6.2 shows $\log \left(I_{D}\right)$ versus $V_{G}$ at fixed drain voltage $\left(V_{D}=-10 \mathrm{~V}\right)$. These data were measured on a FET with a relatively low mobility of $\mu=0.05 \mathrm{~cm}^{2} / \mathrm{Vs}$, i.e. they can be considered as typical. From this measurement we find the subthreshold slope to be $1.6 \mathrm{~V} /$ decade. Normalizing this value to the capacitance of the dielectric gives $28 \mathrm{~V} \cdot \mathrm{nF} /$ decade $\cdot \mathrm{cm}^{2}$. These values are comparable to what is found for the best pentacene TFTs $\left(15-80 \mathrm{~V} \cdot \mathrm{nF} /\right.$ decade $\left.\cdot \mathrm{cm}^{2}[11,19,21]\right)$. 


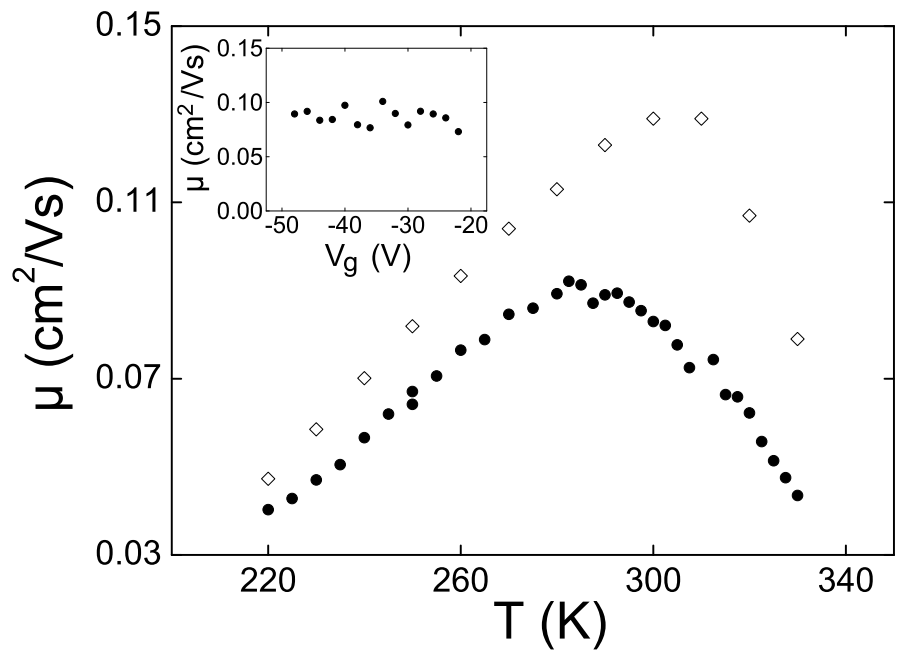

Figure 6.3: Temperature dependence of the field-effect mobility for two different devices, measured at large negative gate voltage. The inset illustrates that at large negative gate voltage, -20 to $-50 \mathrm{~V}$, where the highest mobility is observed, $\mu$ is essentially independent of $V_{G}$.

\subsection{Non-monotonous temperature dependence of mobility}

Further proof of the high quality of the tetracene single-crystal FET is provided by the temperature dependence of the mobility. Temperature dependent measurements were performed in the range $220-330 \mathrm{~K}$, since for $T>330 \mathrm{~K}$ tetracene crystals rapidly sublime at the pressure present in the measurement chamber, and for $T<200 \mathrm{~K}$, a structural phase transition [22] often results in a lowered mobility and in damage to the devices. Fig. 6.3 shows data from two different FETs. The mobility initially increases with lowering temperature from $330 \mathrm{~K}$ to $280-300 \mathrm{~K}$, and then decreases when the temperature is lowered further. Such a non-monotonous temperature dependence is not usually observed in organic TFTs, which typically exhibit a thermally activated decrease of $\mu$ with decreasing $T$, over the entire temperature range investigated [19, 23].

The observed temperature dependence of the mobility is qualitatively similar to the one expected for high-purity organic crystals in the presence of shallow traps, which is given by $\mu \propto T^{-n} \exp \left(-E_{t} / k_{B} T\right)[24,25]$. As long as $E_{t}$ is not much larger than $k_{B} T$, this formula accounts for a non-monotonous temperature dependence of $\mu$ in the temperature range investigated. The observation of a 
maximum mobility at room temperature indicates that $E_{t} \approx 50-100 \mathrm{meV}$ (i.e. a few times $k_{B} T$ at room temperature). This is consistent with the weak gate voltage dependence of the mobility, see the inset of Fig. 6.3, which also points to weak trapping [5].

\subsection{Comparison with TOF experiments}

To obtain more information about the quality of the tetracene crystals and about the FET fabrication process, we have performed Time-of-Flight (TOF) measurements on several thick $(100-200 \mu \mathrm{m})$ tetracene crystals grown by the same technique [26]. For all crystals investigated, the room temperature mobility found in TOF experiments ranges from 0.5 to $0.8 \mathrm{~cm}^{2} / \mathrm{Vs}$, comparable or slightly higher than that obtained from our best FETs. Also the temperature dependence of the mobility is similar to that observed in FET measurements [27]. Finding comparable values for the bulk and the surface mobility [28] suggests that the FET fabrication process does not severely degrade the quality of the crystal surface. We conclude that, presently, the limiting factor for the mobility of the tetracene single-crystal FETs is the quality of the as grown crystals.

\subsection{Conclusion}

In conclusion, we have shown that high-quality organic single-crystal FETs can be realized, whose performance is comparable to the best existing organic thin film transistors. As research on organic thin film FETs has now been going on for many years whereas work on single crystals has just started, we consider this result to be particularly promising for future developments.

\section{References}

[1] B. K. Crone, A. Dodabalapur, R. Sarpeshkar, R. W. Filas, Y.-Y. Lin, Z. Bao, J. H. O’Neill, W. Li, and H. E. Katz, J. Appl. Phys. 89, 5125 (2001)

[2] G. H. Gelinck, T. C. T. Geuns, and D. M. de Leeuw, Appl. Phys. Lett. 77, 1487 (2000)

[3] I. H. Campbell, and D. L. Smith, Solid State Phys. 55,1 (2001)

[4] W. A. Schoonveld, J. Vrijmoeth, and T. M. Klapwijk, Appl. Phys. Lett. 73, 3884 (1998) 
[5] C. D. Dimitrakopoulos, and P. R. L. Malenfant, Adv. Mater. 14, 99 (2002)

[6] W. Warta, and N. Karl, Phys. Rev. B 32, 1172 (1985)

[7] R. Farchioni, and G. Grosso, Organic electronic Materials (Springer-Verlag, Berlin, 2001)

[8] H. Klauk, M. Halik, U. Zschieschang, G. Schmid, W. Radlik, and W. Werner, J. Appl. Phys. 92, 5259 (2002)

[9] S. F. Nelson, Y. -Y. Lin, D. J. Gundlach, and T. N. Jackson, Appl. Phys. Lett. 72, 1854 (1998)

[10] V. Podzorov, V. M. Pudalov, and M. E. Gershenson, Appl. Phys. Lett. 82, 1739 (2003)

[11] V. Podzorov, S. E. Sysoev, E. Loginova, V. M. Pudalov, and M. E. Gershenson, Appl. Phys. Lett. 83, 3504 (2003)

[12] V. Y. Butko, X. Chi, D. V. Lang, and A. P. Ramirez, Appl. Phys. Lett. 83, $4773(2003)$

[13] J. Takeya, C. Goldmann, S. Haas, K. P. Pernstich, B. Ketterer, and B. Batlogg, J. Appl. Phys. 94, 5800 (2003)

[14] M. Ichikawa, H. Yanagi, Y. Shimizu, S. Hotta, N. Suganuma, T. Koyama, and Y. Taniguchi, Adv. Mater. 14, 1272 (2002)

[15] A similar technique has been used for crystals of different molecules, see [13] and [14].

[16] R. A. Laudise, Ch. Kloc, P. G. Simpkins, and T. Siegrist, J. Crystal Growth 187, 449 (1998)

[17] Without RIE cleaning we observe a large spread in mobilities, which are on average much lower than the mobility of FETs made on RIE cleaned substrates. Additionally, we find much larger hysteresis in the electrical measurements and a large negative threshold voltage.

[18] J. Vrijmoeth, R. W. Stok, R. Veldman, W. A. Schoonveld, and T. M. Klapwijk, J. Appl. Phys. 83, 3816 (1998)

[19] D. J. Gundlach, J. A. Nichols, L. Zhou, and T. N. Jackson, Appl. Phys. Lett. 80, 2925 (2002)

[20] For holes, a positive threshold voltage implies conduction through the device in the absence of an applied gate voltage.

[21] Y. -Y. Lin, D. J. Gundlach, S. F. Nelson, and T. N. Jackson, IEEE Trans. Electron Devices 44, 1325 (1997) 
[22] U. Sondermann, A. Kutoglu, H. Bässler, J. Phys. Chem. 89, 1735 (1985)

[23] M. C. J. M. Vissenberg, and M. Matters, Phys. Rev. B 57, 12964 (1998)

[24] E. A. Silinsh, and V. Čápek, Organic Molecular Crystals: Interaction, Localization, and Transport Phenomena (AIP Press, Woodbury, 1994)

[25] N. Karl, K. -H. Kraft, J. Marktanner, M. Münch, F. Schatz, R. Stehle, and H. -M. Uhde, J. Vac. Sci. Technol. A 17, 2318 (1999)

[26] R. W. I. de Boer, M. Jochemsen, T. M. Klapwijk, A. F. Morpurgo, J. Niemax, A. K. Tripathi, and J. Pflaum, J. Appl. Phys. 95, 1196 (2004)

[27] See also J. Berrehar, P. Dellanoy, and M. Schott, Phys. Stat. Sol. (b) 77, K119 (1976)

[28] TOF experiments give a measure of the bulk mobility of charge carriers in the $c$-direction of the crystal, whereas FET experiments probe the mobility of charge carriers at the crystal surface. 


\section{Chapter 7}

\section{Ambipolar $\mathrm{Cu}-$ and Fe-phthalocyanine single-crystal field-effect transistors}

We report the observation of ambipolar transport in field-effect transistors fabricated on single crystals of copper- and iron-phthalocyanine, using gold as a high work-function metal for the fabrication of source and drain electrodes. In these devices, the room-temperature mobility of holes reaches $0.3 \mathrm{~cm}^{2} / \mathrm{Vs}$ in both materials. The highest mobility for electrons is observed for iron-phthalocyanines and is approximately one order of magnitude lower. Our measurements indicate that these values are limited by extrinsic contact effects due to the transistor fabrication and suggest that considerably higher values for the electron and hole mobility can be achieved in these materials.

This Chapter has been published as R. W. I. de Boer, A. F. Stassen, M. F. Craciun, C. L. Mulder, A. Molinari, S. Rogge, and A. F. Morpurgo, Appl. Phys. Lett. 86, 262109 (2005) 


\subsection{Introduction}

Most of the organic transistors that have been fabricated until now have shown unipolar conduction, i.e. they function for only one polarity of the gate voltage [1]. Only very recently, ambipolar device operation has been reported in FieldEffect Transistors (FETs) based on a few different single-component [2] organic molecular semiconductors $[3,4,5,6]$. Some of these ambipolar devices have been used to realize inverter circuits that demonstrate the possibility of implementing CMOS technology [7]. Since CMOS technology is the most efficient strategy towards the fabrication of integrated circuits with better noise margins (i.e. insensitivity to the spread in the parameters of the individual devices) and consuming less power $[3,8]$, this represents a considerable step forward in the field of plastic electronics. Particularly important is that the implementation of plastic CMOS integrated circuits can be realized using a single molecular semiconductor rather than two different materials, since this has the potential to considerably simplify the device fabrication processes. So far, however, the number of single-component molecular semiconductors for which ambipolar FET operation has been reported is rather limited.

In this Chapter we report the investigation of field-effect transistors fabricated on single crystals of copper- and iron-phthalocynanines (Cu- and Fe-Pc's), using gold as a high work function material for the fabrication of the source and drain electrodes. Electrical measurements performed in high-vacuum demonstrate the occurrence of both hole and electron conduction. From the electrical characteristics of these first organic single-crystal FET we extract room-temperature mobility values for holes reaching $0.3 \mathrm{~cm}^{2} / \mathrm{Vs}$ for both materials. The typical electron mobility values are different for $\mathrm{FePc}$ and $\mathrm{CuPc}$. In $\mathrm{FePc}$ the electron mobility reaches $0.03 \mathrm{~cm}^{2} / \mathrm{Vs}$ whereas, in $\mathrm{CuPc}$ it is typically lower than $10^{-3}$ $\mathrm{cm}^{2} / \mathrm{Vs}$. These values are limited by device non-idealities of extrinsic origin, which originate from difficulties in the FET assembly and which clearly manifest themselves in the transistor electrical characteristics. We expect that, upon improving the device assembly procedure, considerably higher electron and hole mobility can be reached in copper- and iron-phthalocyanine ambipolar FETs.

\subsection{Processing-related specifications of $\mathrm{CuPc}$ and FePc crystals}

The devices used in our studies have been fabricated by means of electrostatic bonding of very thin $(\sim 1 \mu \mathrm{m}$ thick $)$, flexible crystals to a substrate onto which 
the FET circuitry had been previously fabricated. The substrate is highly-doped $\mathrm{Si}$, covered by a $100 \mathrm{~nm}$ thick layer of $\mathrm{SiO}_{2}$ that serves as the gate insulator, which is cleaned by Reactive Ion Etching (RIE) in an oxygen plasma prior to bonding with the crystal. The details of the technique and of the crystal growth are similar to those used for the fabrication of tetracene and rubrene single-crystal FETs [9]. From X-ray diffraction measurements we find that the structure of both $\mathrm{CuPc}$ and FePc crystals, which were grown from vapor phase [10], is the known $\beta$-form [11]. The molecules are arranged in layers with a herringbone structure and they form columnar stacks aligned along the crystalline b-direction [12]. All crystals used in this work are needle-shaped and their long direction corresponds to the stacking direction in the columns.

Contrary to what happens for rubrene or tetracene single crystals [9], for which the width of very thin crystals can easily exceed $1 \mathrm{~mm}$, we have found that the width of thin and flexible $\mathrm{CuPc}$ and FePc single crystals that are capable of bonding to rigid substrates is typically smaller than $100 \mu \mathrm{m}$ (see inset in Fig. 7.1). In addition, very thin crystals are fragile and easily crack along the crystalline $b$-axis. These properties of the $\mathrm{CuPc}$ and $\mathrm{FePc}$ crystals substantially increase the difficulties involved in the crystal handling and in the device assembly, often resulting in damage to the crystal or in a poorer adhesion of the crystals to the substrate and to the contacts. In spite of these difficulties, we have successfully fabricated and measured a large number of FETs on FePc and CuPc. All of the devices fabricated using $\mathrm{FePc}$ and approximately half of the devices fabricated using $\mathrm{CuPc}$ exhibited ambipolar transport.

\subsection{Observations of ambipolar transport}

The electrical characterization of the devices has been performed in a vacuum chamber (pressure $\approx 10^{-7}$ mbar) and in the dark. All the data shown here were taken in a two terminal configuration, using a HP4156 parameter analyzer, at room temperature. We found that heating of the device in-situ up to a temperature of $450 \mathrm{~K}$ for a period of several hours results in a clear improvement of the electrical characteristics (e.g. reduction of hysteresis) and in a substantial enhancement of the electron current. This behavior has been observed before and is attributed to thermally-induced de-sorption of oxygen and water vapor present in the crystals $[3,13]$ and acting as traps for electrons [14]. We have also noticed that heating the device for much longer periods (days) results in further smaller improvements of the transistor characteristics, e.g. a factor-of-two enhancement of the mobility of both electrons and holes. This phenomenon is likely to origi- 
nate from the lowering of the contact resistance due to a better adhesion between crystal and electrodes, or from the annealing of structural defects.

Fig. 7.1 shows logarithmic plots of the source-drain current $I_{D}$ measured as a function of gate voltage $V_{G}$, for a fixed value of source-drain voltage $V_{D}$, in a $\mathrm{FePc}$ and in a $\mathrm{CuPc}$ device. It is apparent that the measured current increases with increasing $V_{G}$ for both negative and positive polarity. This demonstrates the occurrence of ambipolar transport in these materials. In general, we observe that at large gate voltage, and under comparable biasing conditions, the electron current is normally lower than the hole current (approximately one-to-two orders of magnitude).

The transistor characteristics of a FePc device measured by sweeping the source-drain voltage at different, fixed values of $V_{G}$ are shown in Fig. 7.2. These measurements illustrate that the typical transistor behavior is observed for both negative and positive gate voltage and confirm the occurrence of ambipolar operation. Overall, the observed behavior is very similar to that exhibited by recently reported, ambipolar organic FETs, realized on thin-films of different organic semiconductors [3].

\subsection{Intrinsic and extrinsic effects of high work- function $\mathrm{Au}$ contacts}

The mobility of electrons and holes in different FETs has been estimated at room temperature from the linear regime of the device operation. We find a rather large spread in the measured mobility values. For FePc hole and electron mobilities were found in the range $10^{-3}-3 \cdot 10^{-1} \mathrm{~cm}^{2} / \mathrm{Vs}$ and $5 \cdot 10^{-4}-3$. $10^{-2} \mathrm{~cm}^{2} / \mathrm{Vs}$, respectively. For $\mathrm{CuPc}$, the measured values for the hole mobility are in the same range as in $\mathrm{FePc}$, whereas the values for electron mobility are typically lower $\left(10^{-5}-10^{-3} \mathrm{~cm}^{2} / \mathrm{Vs}\right)$. We attribute the large spread of mobility to the presence of contact effects that are visible in the electrical characteristics of even the best devices (see Fig. 7.3). In this regard, the electrical behavior of these $\mathrm{FePc}$ and $\mathrm{CuPc}$ FETs is different from that observed in tetracene [15] and rubrene $[16,17,18]$ single-crystal FET, which exhibit a considerably smaller spread in mobility values. Nevertheless, the best mobility values that we have found for both electron and holes already compare well to the highest mobility values reported for ambipolar thin-film organic FETs.

Since contact limitations in FePc and $\mathrm{CuPc}$ single-crystal FETs are visible for both holes and electrons even though the work function of gold is ideally aligned to the HOMO of the molecules [19], we conclude that these contact effects are 

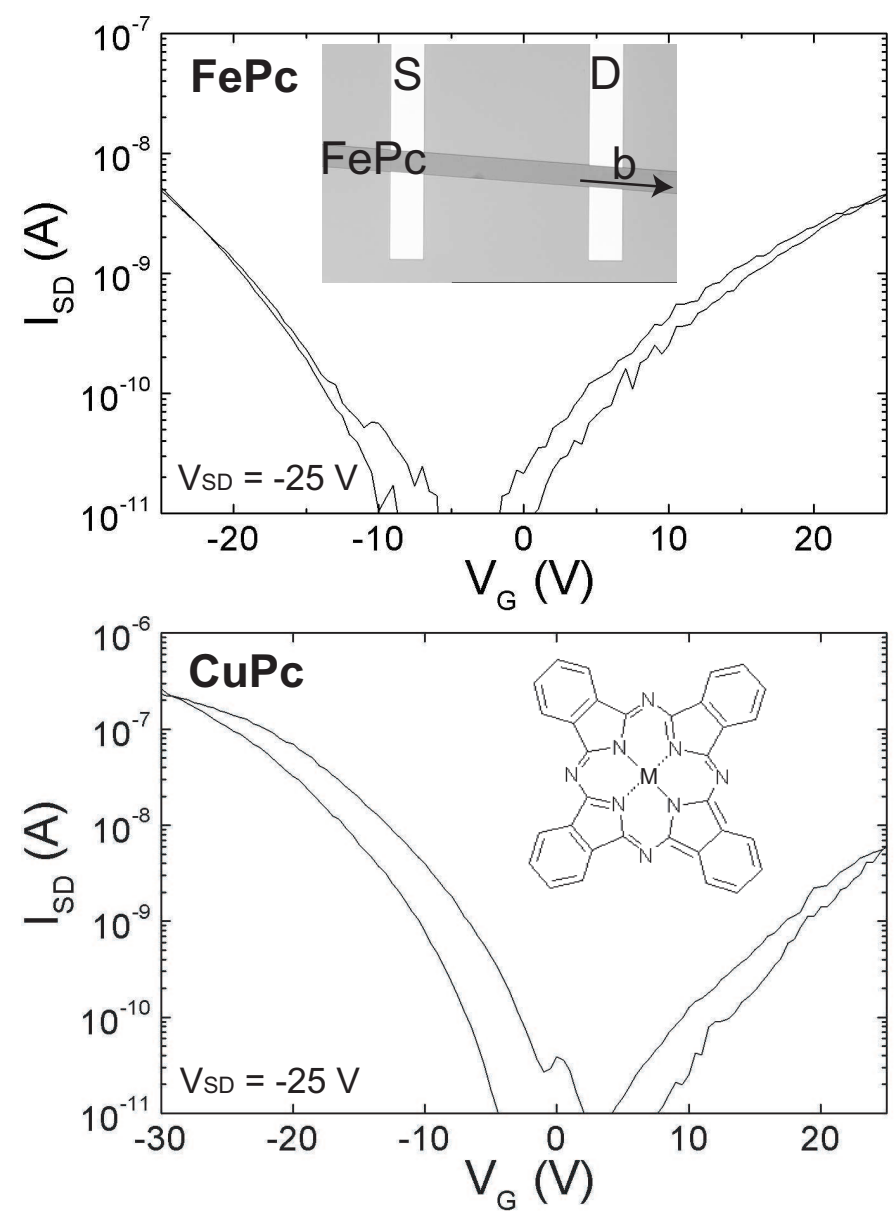

Figure 7.1: Examples of transfer characteristics illustrating ambipolar transport in $\mathrm{FePc}$ and $\mathrm{CuPc}$ single-crystal FETs with gold electrodes. At negative $V_{G}$ the holecurrent is visible, and at positive $V_{G}$ there is a clear electron-current. The inset in the upper plot shows a microscope picture of an FePc FET, with a distance of $500 \mu \mathrm{m}$ between the source $(\mathrm{S})$ and the drain $(\mathrm{D})$. The arrow indicates the crystalline $b$-axis. The inset in the lower plot shows the molecular structure of MPc's.

largely of extrinsic origin, due to the FET assembly process (e.g. imperfect adhesion of the crystal onto the metal contact). Note, however, that whereas the values for the hole mobility are approximately the same for the two different phthalocyanines, the mobility of electrons is systematically higher in FePc than in CuPc. This is consistent with the HOMO-LUMO gap of FePc being smaller than that of $\mathrm{CuPc}$ [19], which allows a more efficient electron injection in FePc than in $\mathrm{CuPc}$. Thus, in short, the data suggest that even though extrinsic contact effects are present and limit the measured value of the hole mobility to $0.3 \mathrm{~cm}^{2} / \mathrm{Vs}$, the electron current is also significantly limited by the intrinsic misalignment 

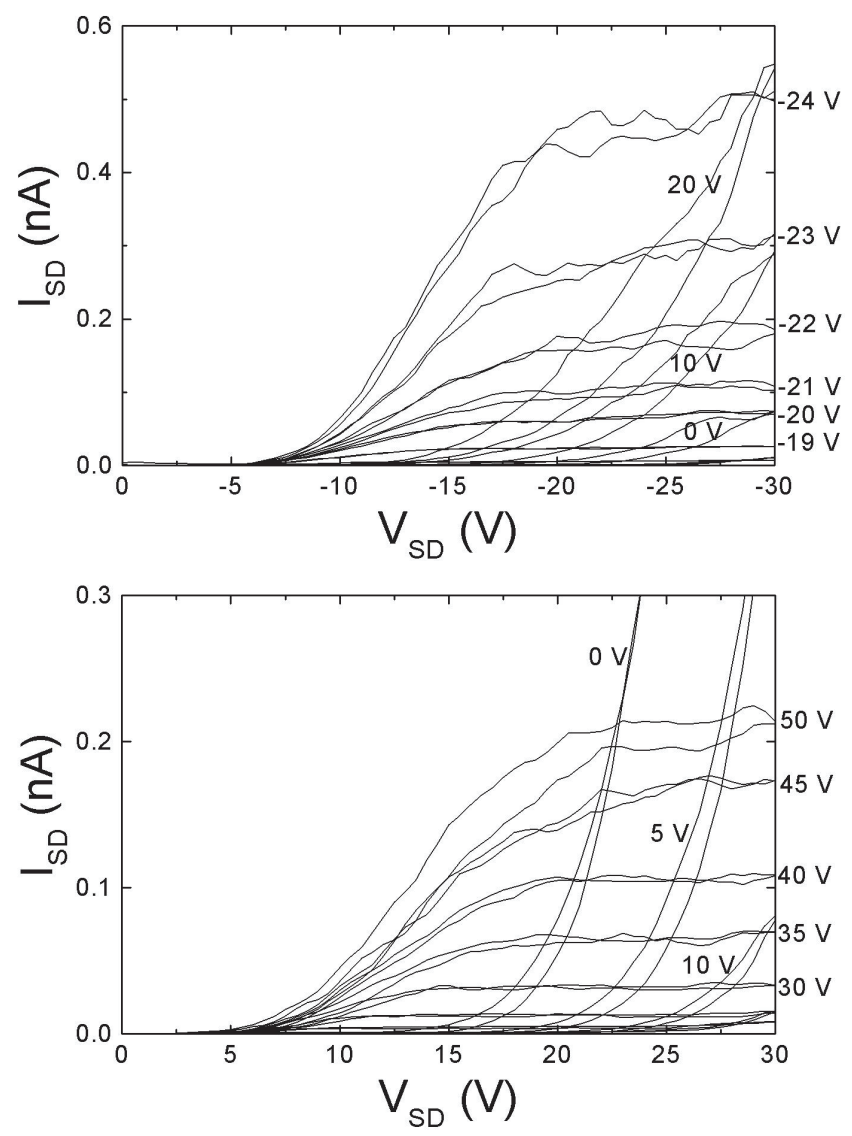

Figure 7.2: Current-voltage characteristics of a FePc single-crystal FET, demonstrating ambipolar operation. The $V_{G}$-values are indicated in the plots.

between the gold work function and the molecular level for electron transport in the material [3].

In comparing the behavior of our single-crystal FETs with thin-film FETs made with the same materials, it is interesting to note that ambipolar transport in $\mathrm{CuPc}$ thin-film transistors has been observed only very recently in devices using Calcium electrodes [6], whose work function is more suitable than gold for electron injection. Even though gold contacted $\mathrm{CuPc}$ thin-film FETs have been extensively studied in the past [20], ambipolar operation has never been reported in those devices. This suggest that the structural quality of the organic semiconductor (a major difference between thin-film and single-crystal devices) is also a relevant factor for the observation of ambipolar FET operation [14]. 


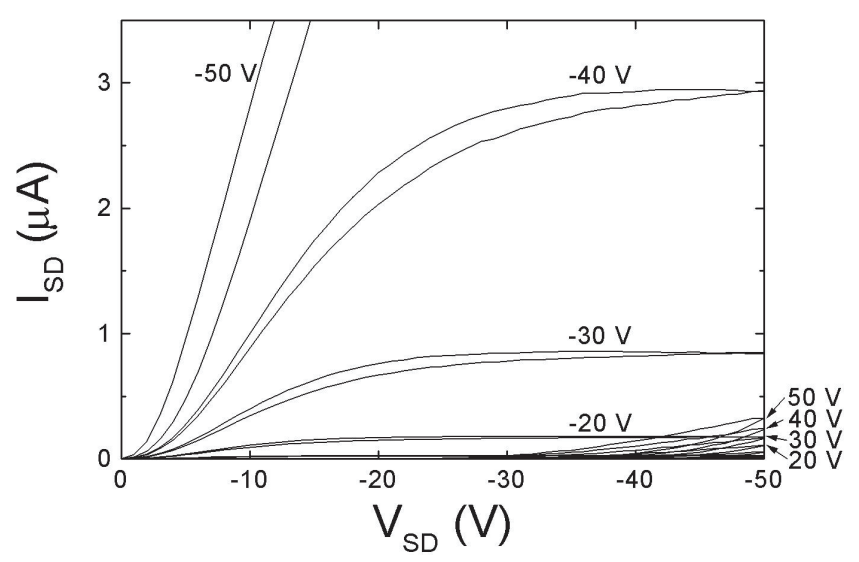

Figure 7.3: Current-voltage characteristics of one of the best FePc single-crystal FETs, with $\mu_{\text {hole }}=0.3 \mathrm{~cm}^{2} /$ Vs. Even in the best devices a clear non-linearity at low $V_{D}$ is observed that indicates the presence of contact non-idealities.

\subsection{Opportunities for further research}

Finally, together with previous experiments on thin-film FETs of different phthalocyanines in which both p- and n-type operation has been observed depending on the atmosphere in which the devices are operated [21, 22], our results suggest that it will be possible to observe ambipolar transport in many different phthalocyanine-based semiconductors. This is of interest for fundamental investigations, since the molecular orbitals occupied by the charge carriers in many different phathalocyanines are different, whereas the structure of the molecules (as well as that of their crystals) is essentially identical. For instance, in $\mathrm{CuPc}$ as well as in other metal-Pc's (e.g. $\mathrm{MgPc}$ or $\mathrm{ZnPc}$ ), the field-induced electrons occupy orbitals that are centered on the Pc ligands (the doubly-degenerate $2 e_{g}$ orbital). In $\mathrm{FePc}$ (and similarly in $\mathrm{CoPc}$ ), on the contrary, electrons occupy orbitals mainly centered on the metal atom [19]. Thus, the comparative studies of electron transport in different metal-Pc single-crystal FETs enables the investigation of the relation between the conduction properties of organic semiconductors and the electronic properties of the orbitals of the constituent molecules. For these studies, we are working to improve of the quality of $\mathrm{MPc}$ single-crystal FETs.

\section{References}

[1] C. D. Dimitrakopoulos, and P. R. L. Malenfant, Adv. Mater. 14, 99 (2002) 
[2] Ambipolar organic transistors based on more than one component exist for several years already: see A. Dodabalapur, H. E. Katz, L. Torsi, and R. C. Haddon, Appl. Phys. Lett. 68, 1108 (1996)

[3] E. J. Meijer, D. M. de leeuw, S. Setayesh, E. van Veenendaal, B. -H. Huisman, P. W. M. Blom, J. C. Hummelen, U. Scherf, and T. M. Klapwijk, Nature Mater. 2, 678 (2003)

[4] R. J. Chesterfield, C. R. Newman, T. M. Pappenfus, P. C. Ewbank, M. H. Haukaas, K. R. Mann, L. L. Miller, and C. D. Frisbie, Adv. Mater. 15, 1278 (2003)

[5] Y. Kunugi, K. Takimiya, N. Negishi, T. Otsubo, and Y. Aso, J. Mater. Chem. 14, 2840 (2004)

[6] T. Yasuda, and T. Tsutsui, Chem. Phys. Lett. 402, 395 (2005)

[7] A. D. Anthopoulos, D. M. de Leeuw, A. Cantatore, S. Setayesh, E. J. Meijer, C. Tanase, J. C. Hummelen, and P. W. M. Blom, Appl. Phys. Lett. 85, 4205 (2004)

[8] B. Crone, A. Dodabalapur, Y. -Y. Lin, R. W. Filas, Z. Bao, A. LaDuca, R. Sarpeshkar, H. E. Katz, and W. Li, Nature 403, 521 (2000)

[9] R. W. I. de Boer, M. E. Gershenson, A. F. Morpurgo, and V. Podzorov, Phys. Stat. Sol. 201, 1302 (2004)

[10] R.A. Laudise, Ch. Kloc, P.G. Simpkins, and T. Siegrist, J. Crystal Growth 187, 449 (1998)

[11] R. D. Gould, Coord. Chem. Rev. 156, 237 (1996)

[12] R. Zeis, T. Siegrist, and Ch. Kloc, Appl. Phys. Lett. 86, 022103 (2005)

[13] D. M. de Leeuw, M. M. J. Simenon, A. R. Brown, and R. E. F. Einerhand, Synth. Met. 87, 53 (1997)

[14] This is probably the reason why electron conduction was not observed in $\mathrm{CuPc}$ single-crystal transistors by Zeis et al. [12]. They used as the gatedielectric a parylene thin-film, which is inpenetrable to oxygen and water molecules and thus encapsulates them in the crystal.

[15] R. W. I. de Boer, T. M. Klapwijk, and A. F. Morpurgo, Appl. Phys. Lett. 83, $4345(2003)$

[16] V. Podzorov, V.M. Pudalov, and M.E. Gershenson, Appl. Phys. Lett. 82, 1739 (2003)

[17] V. Podzorov, E. Menard, A. Borissov, V. Kiryukhin, J. A. Rogers, and M. E. Gershenson Phys. Rev. Lett. 93, 086602 (2004) 
[18] A. F. Stassen, R. W. I. de Boer, N. N. Iosad, and A. F. Morpurgo, Appl. Phys. Lett. 85, 3899 (2004)

[19] M. -S. Liao, and S. Scheiner, J. Chem. Phys. 114, 9780 (2001)

[20] Z. Bao, A. J. Lovinger, A. Dodabalapur, Appl. Phys. Lett. 69, 3066 (1996)

[21] G. Guillaud, M. Al Sadoun, M. Maitrot, J. Simon, and M. Bouvet, Chem. Phys. Lett. 167, 503 (1990)

[22] H. Tada, H. Touda, M. Takada, and K. Matsushige, Appl. Phys. Lett. 76, $873(2000)$ 


\section{Chapter 8}

\section{Influence of the gate leakage current on the stability of organic single-crystal field-effect transistors}

We investigate the effect of a small leakage current through the gate insulator on the stability of organic single-crystal Field-Effect Transistors (FETs). We find that, irrespective of the specific organic molecule and dielectric used, leakage current flowing through the gate insulator results in an irreversible degradation of the single-crystal FET performance. This degradation occurs even when the leakage current is several orders of magnitude smaller than the source-drain current. The experimental data indicate that a stable operation requires the leakage current to be smaller than $10^{-9} \mathrm{~A} / \mathrm{cm}^{2}$. Our results also suggest that gate leakage currents may determine the lifetime of thin-film transistors used in applications.

This Chapter has been published as R. W. I. de Boer, N. N. Iosad, A. F. Stassen, T. M. Klapwijk, and A. F. Morpurgo, Appl. Phys. Lett. 86, 032103 (2005) 


\subsection{Introduction}

The study of organic semiconductor transistors aims at the development of organic electronics, for its advantages of being flexible, cheap and suitable for largearea production $[1,2]$. So far, considerable research effort has been focused on the optimization of the organic layer to improve the performance of thin-film transistors $[3,4,5]$. Much less attention has been devoted to other important device aspects, such as, for instance, the choice of the gate insulator.

Recent work has demonstrated that the gate insulator plays an important role in determining the device performance [6]. In particular, it has been shown that in polymer transistors the mobility of charge carriers is systematically larger the lower the dielectric constant of the gate insulator. This implies that the use of low- $\epsilon$ dielectrics will result in a higher device switching speed. In view of this result, it appears useful to investigate systematically how different properties of the gate insulator affect the behavior of organic transistors.

In this Chapter we use organic single-crystal FETs [7] to investigate how a small leakage current through the gate insulator affects the stability of the device operation. Specifically, we have investigated the behavior of organic single-crystal FETs of different molecules (tetracene, rubrene, perylene) in combination with different dielectrics $\left(\mathrm{Ta}_{2} \mathrm{O}_{5}, \mathrm{ZrO}_{2}\right.$, and $\left.\mathrm{SiO}_{2}\right)$. We find that, irrespective of the specific molecule and dielectric used, leakage current flowing through the gate insulator results in an irreversible degradation of the single-crystal FET operation. The degradation is not due to the electrical breakdown of the insulating layer and it also occurs when the leakage current is several orders of magnitude smaller than the source-drain current. From the experimental data, we conclude that a stable operation of organic single-crystal FETs requires the current leaking to the FET channel to be smaller than $10^{-9} \mathrm{~A} / \mathrm{cm}^{2}$.

\subsection{Characterization of sputtered $\mathrm{Ta}_{2} \mathrm{O}_{5}$ and $\mathrm{ZrO}_{2}$ films}

The fabrication of the single-crystal FETs used in this work is based on electrostatic bonding of an organic single crystal to a dielectric surface, with prefabricated source, drain and gate contacts. The details are essentially identical to what has been described in Ref. [8]. Whereas in Ref. [8] only thermally grown $\mathrm{SiO}_{2}$ was used as gate insulator, here we have also used sputtered layers of $\mathrm{Ta}_{2} \mathrm{O}_{5}$ and $\mathrm{ZrO}_{2}$ deposited in different ways. For both $\mathrm{Ta}_{2} \mathrm{O}_{5}$ and $\mathrm{ZrO}_{2}$ we have investigated FETs in which the dielectric layers were sputtered from ceramic targets 


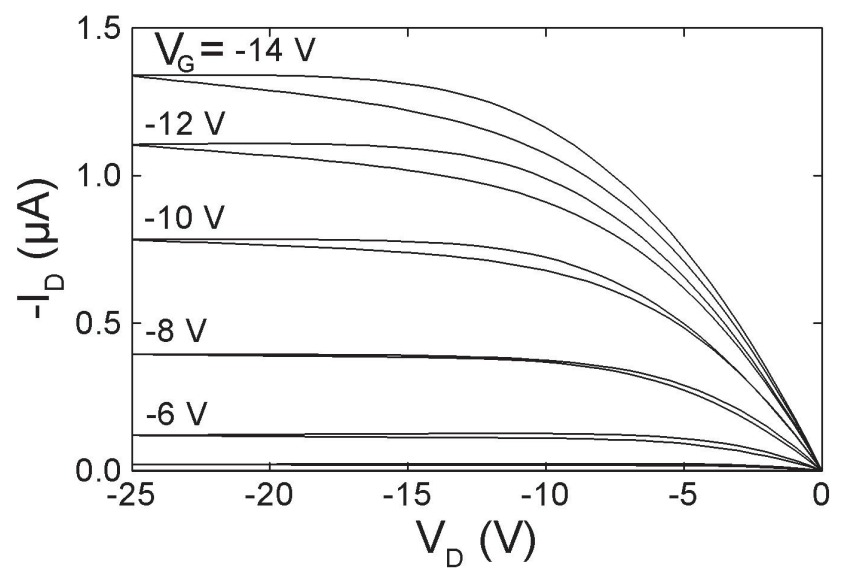

Figure 8.1: Current-voltage characteristics of an organic single-crystal FET, with tetracene as the organic molecule and type $\mathrm{I} \mathrm{Ta}_{2} \mathrm{O}_{5}$ as the gate insulator.

(hereafter referred to as "type I" oxides; see Ref. [9] for details). For $\mathrm{Ta}_{2} \mathrm{O}_{5}$, we have also investigated the behavior of FETs fabricated on layers sputtered from a metallic target, in the presence of oxygen in the sputtering gas, as described in Ref. [10] (hereafter referred to as "type II" $\mathrm{Ta}_{2} \mathrm{O}_{5}$ ). For all FETs discussed here the sputtered oxide layers were approximately $350 \mathrm{~nm}$ thick.

The electrical properties of all the different dielectric layers were characterized by capacitance and $I-V$ measurements (see [9]). From these measurements we obtain a dielectric constant $\epsilon=25$ for $\mathrm{Ta}_{2} \mathrm{O}_{5}$ (both types) and 23 for $\mathrm{ZrO}_{2}$, as expected. The breakdown field is comparable for all layers and typically equal to $E_{\mathrm{bd}} \simeq 4-6 \mathrm{MV} / \mathrm{cm}$. The leakage current, on the contrary, is different for the different layers. Specifically, at a voltage corresponding to approximately half the breakdown field, the leakage through type $\mathrm{I} \mathrm{Ta}_{2} \mathrm{O}_{5}$ is typically in the order of $10^{-6} \mathrm{~A} / \mathrm{cm}^{2}$, slightly higher than that through $\mathrm{ZrO}_{2}, 10^{-7}-10^{-6} \mathrm{~A} / \mathrm{cm}^{2}$ and much higher than that flowing through type II $\mathrm{Ta}_{2} \mathrm{O}_{5},<10^{-9} \mathrm{~A} / \mathrm{cm}^{2}$.

\subsection{Anomalous electrical behavior of $\mathrm{Ta}_{2} \mathrm{O}_{5}$ and $\mathrm{ZrO}_{2}$ FETs}

Electrical characterization of all devices is performed in the dark, at room temperature and under vacuum $\left(10^{-7}\right.$ mbar $)$, using a HP4156A Semiconductor Parameter Analyzer, in a two-terminal configuration. Fig. 8.1 shows the transistor characteristics of a tetracene FET with type $\mathrm{I} \mathrm{Ta}_{2} \mathrm{O}_{5}$ as gate dielectric. Upon a superficial inspection, these characteristics resemble those of tetracene FETs on $\mathrm{SiO}_{2}$ (see Ref. [8]). The typical mobility values in $\mathrm{Ta}_{2} \mathrm{O}_{5}$ devices range from 
0.02 to $0.08 \mathrm{~cm}^{2} / \mathrm{Vs}$, smaller than in devices on $\mathrm{SiO}_{2}$, as expected in view of the larger dielectric constant of $\mathrm{Ta}_{2} \mathrm{O}_{5}$ (see Chapter 9). A first unexpected difference is however visible in Fig. 8.1, as the transistor characteristics exhibit some hysteresis, not normally observed in high-quality tetracene single-crystal FETs on $\mathrm{SiO}_{2}$. A much more striking difference between the type $\mathrm{I} \mathrm{Ta}_{2} \mathrm{O}_{5}$ and $\mathrm{ZrO}_{2}$ FETs and the $\mathrm{SiO}_{2}$ FETs is clearly apparent when looking at the $V_{G}$ dependence of $I_{D}$ measured at a fixed $V_{D}$ value.

Fig. 8.2a shows the data obtained from a type I $\mathrm{Ta}_{2} \mathrm{O}_{5}$ FET. Contrary to the usual behavior observed in tetracene FETs on $\mathrm{SiO}_{2}$ (Fig. 8.2c), i.e. $I_{D}$ increasing linearly with $V_{G}$, the source-drain current in type $\mathrm{I} \mathrm{Ta}_{2} \mathrm{O}_{5}$ FETs increases, reaches a maximum and then decreases again. The decrease results in the full suppression of the source-drain current, unless dielectric breakdown of the insulator occurs first. This behavior has been observed in all fabricated devices, irrespective of the organic material used (tetracene, rubrene, perylene).

For a tetracene FET on $\mathrm{ZrO}_{2} I_{D}-V_{G}$ curves measured at fixed $V_{D}$ are shown in Fig. 8.2b. Again, the $I_{D}-V_{G}$ relation is markedly non-linear, although full suppression of the source-drain current is not reached. The shape of the non-linearity is different from that observed in type $\mathrm{I} \mathrm{Ta}_{2} \mathrm{O}_{5}$ devices and is characteristic for our $\mathrm{ZrO}_{2}$ transistors. Also for $\mathrm{ZrO}_{2}$ FETs, the behavior of the $I_{D}-V_{G}$ is similar when crystals of different organic materials are used.

The anomalous behavior reproducibly exhibited by type $\mathrm{I} \mathrm{Ta}_{2} \mathrm{O}_{5}$ and $\mathrm{ZrO}_{2}$ FETs originates from irreversible device degradation. Specifically, we observe that, for every device studied, repeating the measurement of the $I_{D}-V_{G}$ curve systematically results in lower measured values of $I_{D}$ (see Fig. 8.2b). This is due to both a decrease in the device mobility and a shift of the threshold voltage to larger negative values. For those type $\mathrm{I} \mathrm{Ta}_{2} \mathrm{O}_{5}$ transistors in which the increase in $V_{G}$ is sufficient to fully suppress the source-drain current (see Fig. 8.2a), no fieldeffect induced current is ever observed after the first measurement, indicating that the degradation of the device is complete. Attempts to recover the device by biasing the gate positively, or by leaving the device unbiased for several days, were unsuccessful.

\subsection{Performance degradation due to small gate- leakage currents}

Inspection of the degraded transistors using an optical microscope does not reveal any visible change in the device. The bulk of the crystal, the dielectric layer, and the FET circuitry appear to have all remained intact and the crystal is still well 

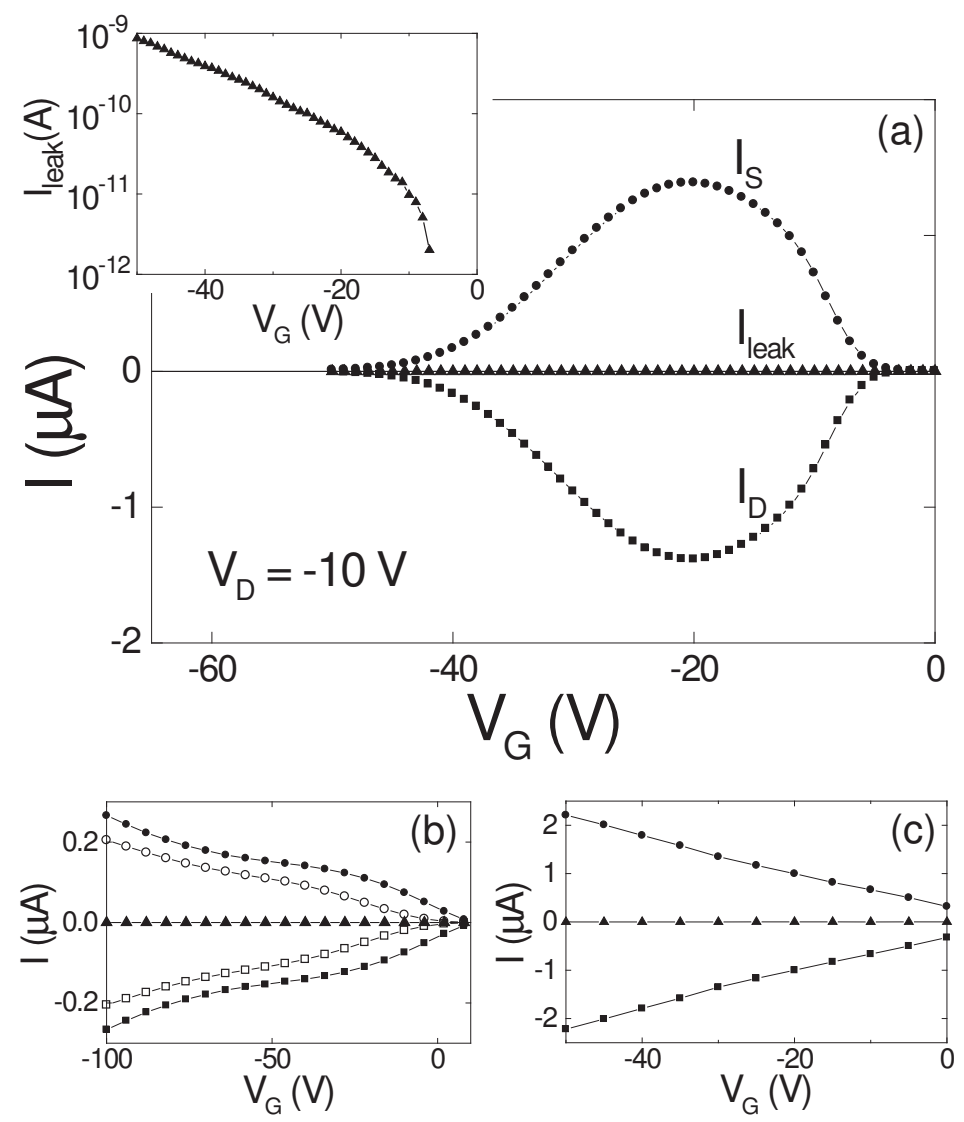

Figure 8.2: (a) Transfer characteristics of a FET on $\mathrm{Ta}_{2} \mathrm{O}_{5}\left(V_{D}=-10 \mathrm{~V}\right)$. The source and drain current $\left(I_{S}\right.$ and $\left.I_{D}\right)$, are equal and opposite, since the leakage current $I_{\text {leak }}$ is orders of magnitude smaller than $I_{D}$ (see inset). Device degradation is responsible for the non-monotonic $I_{D}-V_{G}$ curve, resulting in the full suppression of $I_{D}$ at high $V_{G}$. (b) Transfer characteristics of a tetracene FET on $\mathrm{ZrO}_{2}\left(V_{D}=-10 \mathrm{~V}\right)$. The $I_{D}-V_{G}$ relation is non-linear and $I_{D}$ and $I_{S}$ are lower in the second $V_{G}$ sweep (open circles) than in the first sweep (closed circles). $I_{\text {leak }}$ (triangles) is much smaller than $I_{D}$ and $I_{S}$. Note that the shape of the $I_{D}-V_{G}$ curves is characteristic for $\mathrm{ZrO}_{2}$ and differs from that of $\mathrm{Ta}_{2} \mathrm{O}_{5}$. For comparison, (c) shows the transfer characteristics of a tetracene FET on $\mathrm{SiO}_{2}$ for which $I_{S}$ and $I_{D}$ are linearly related to $V_{G}\left(V_{D}=-10 \mathrm{~V}\right)$.

bonded to the substrate. This suggests that the device degradation is confined to the first layers of the organic material at the interface with the dielectric.

To determine the cause of device degradation it is revealing to compare the behavior of single-crystal FETs fabricated using type I and type II $\mathrm{Ta}_{2} \mathrm{O}_{5}$. Fig. 8.3 shows the results of $I_{D}-V_{G}$ sweeps for two rubrene single-crystal FETs fabricated using the two different oxides. Similar to what we have shown for the tetracene FETs in Fig. 8.2, the FET on type I oxide exhibits a non-linear, non-monotonic 
$I_{D}-V_{G}$ relation up to the dielectric breakdown voltage (at $V_{G} \approx-80 \mathrm{~V}$ for this sample). Conversely, for the rubrene FET on type II $\mathrm{Ta}_{2} \mathrm{O}_{5}, I_{D}$ scales linearly with $V_{G}$ in a large range of values above the threshold voltage, up to the maximum voltage reached in the experiment $\left(V_{G}=-100 \mathrm{~V}\right.$, corresponding to a charge density of $\simeq 4 \cdot 10^{13}$ holes $\left./ \mathrm{cm}^{2}\right)$. For this FET, multiple measurements of the $I_{D}-V_{G}$ curve reproducibly give the same result. Note also, in the inset of Fig. 8.3, that the transfer characteristics of the rubrene FET on type $\mathrm{II} \mathrm{Ta}_{2} \mathrm{O}_{5}$ are fully hysteresis-free, as is also typical for high-quality transistors fabricated on $\mathrm{SiO}_{2}$. In short, contrary to what happens to devices based on type $\mathrm{I} \mathrm{Ta}_{2} \mathrm{O}_{5}$, for FETs fabricated on type II $\mathrm{Ta}_{2} \mathrm{O}_{5}$ device degradation does not occur. Since the main difference between type I and type II $\mathrm{Ta}_{2} \mathrm{O}_{5}$ layers is the much higher level of leakage current observed in the type I layers, this observation suggests that the current leaking through the gate insulator is the cause for the device degradation.

To further investigate the origin of the FET degradation, we have also studied FETs fabricated on bilayers consisting of a $350 \mathrm{~nm}$ thick layer of type $\mathrm{I} \mathrm{Ta}_{2} \mathrm{O}_{5}$ $\left(\mathrm{ZrO}_{2}\right)$ covered with a $25 \mathrm{~nm}$ thin top layer of $\mathrm{ZrO}_{2}$ (type I $\mathrm{Ta}_{2} \mathrm{O}_{5}$ ), so that the organic crystal is in contact with the thin top layer. For these FETs, the shape of the $I_{D}-V_{G}$ curve is similar to that observed in FETs where the thin top layer is not present. These experiments indicate that the details of the device degradation are determined by the thick oxide layer and not by the material directly in contact with the organic crystals. This observation rules out the possibility that a chemical reaction between molecules and dielectric material is causing the device degradation and confirms the role of the leakage current, since in these oxide bi-layers it is the thick layer that determines the magnitude of $I_{\text {leak }}$.

We conclude that damage to the organic crystal induced by current leaking through the gate insulator is the cause for the device degradation [11]. This conclusion is further supported by the absence of degradation in single-crystal FETs fabricated on $\mathrm{SiO}_{2}$, in which the leakage current is undetectably small. It is also consistent with the larger degradation observed in type $\mathrm{I} \mathrm{Ta}_{2} \mathrm{O}_{5}$ FETs as compared to $\mathrm{ZrO}_{2}$ devices, since the leakage current through $\mathrm{ZrO}_{2}$ is typically almost an order of magnitude less than in type $\mathrm{I} \mathrm{Ta}_{2} \mathrm{O}_{5}$.

It is worth noting that degradation occurs even when the leakage current is several orders of magnitude lower than the source-drain current. Specifically, our data quantitatively show that in organic single-crystal FETs gate leakage currents larger than approximately $10^{-9} \mathrm{~A} / \mathrm{cm}^{2}$ [12] systematically result in irreversible device degradation. This conclusion poses a clear constraint on the design of properly functioning single-crystal FETs. It is possibly also relevant for organic thin-film transistors, as it suggests that the gate leakage current is an important factor in determining the device lifetime. 


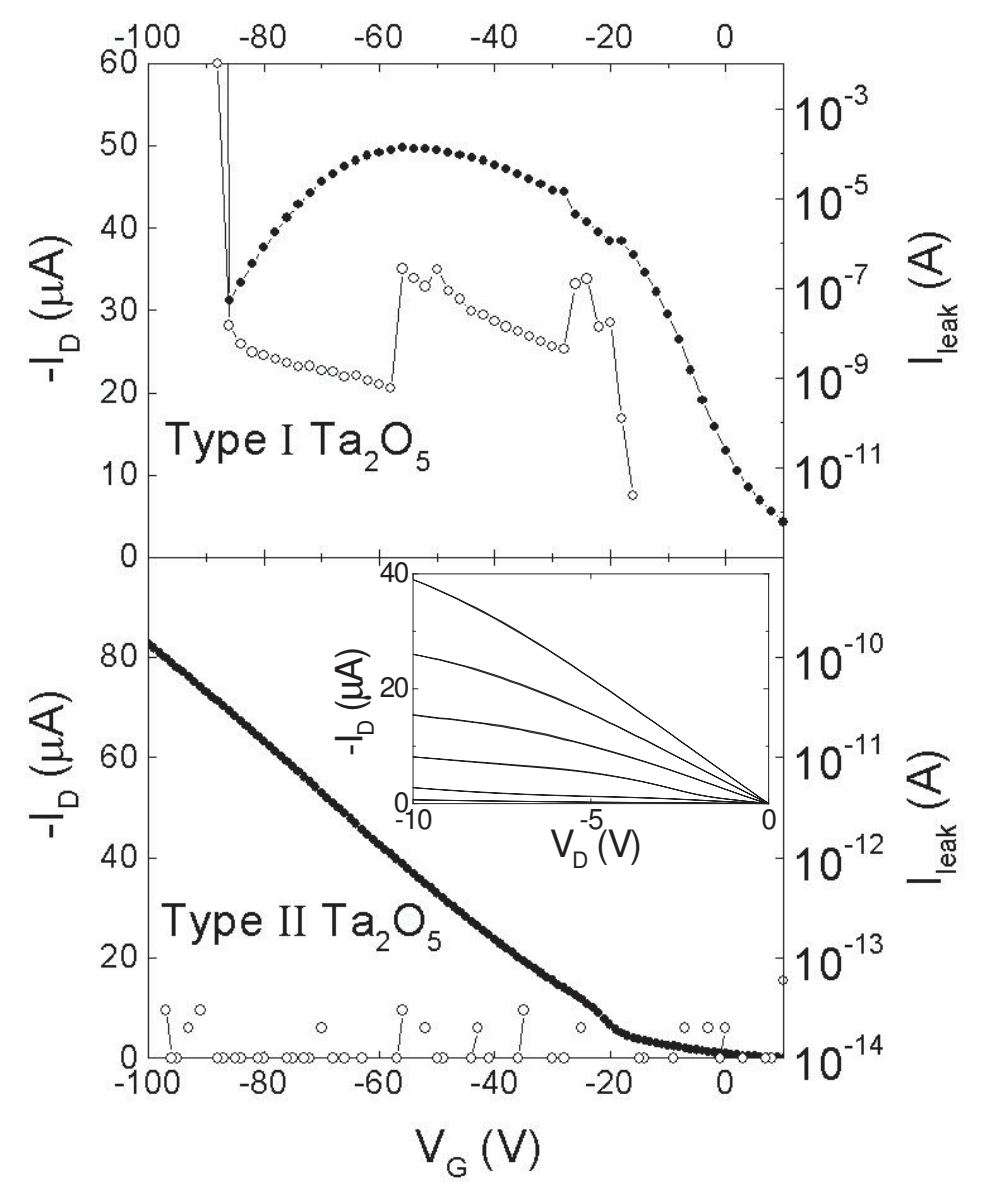

Figure 8.3: $I_{D}-V_{G}$ curves (closed symbols) of two rubrene FETs on type $\mathrm{I} \mathrm{Ta}_{2} \mathrm{O}_{5}$ (upper panel) and type II $\mathrm{Ta}_{2} \mathrm{O}_{5}$ (lower panel) $\left(V_{D}=-10 \mathrm{~V}\right.$ in both cases). The open symbols represent the leakage current. The degradation-induced, non-monotonic behavior of the $I_{D}-V_{G}$ curve observed for the type $\mathrm{I} \mathrm{Ta}_{2} \mathrm{O}_{5}$ FET is absent in the type II $\mathrm{Ta}_{2} \mathrm{O}_{5}$ device. Note that the leakage current in the two devices differs by orders of magnitude, despite the comparable crystal surface area $\left(\sim \mathrm{mm}^{2}\right)$ and $\mathrm{Ta}_{2} \mathrm{O}_{5}$ thickness $(\sim 350 \mathrm{~nm})$. The inset shows that FETs on type II $\mathrm{Ta}_{2} \mathrm{O}_{5}$ exhibit hysteresis-free electrical characteristics.

\subsection{Conclusion}

In conclusion, we have shown that leakage current from the gate electrode causes irreversible degradation of organic single-crystal FETs, even when it is orders of magnitude smaller than the source-drain current. This poses a clear constraint for the design of single-crystal transistors currently used to investigate the intrinsic electronic properties of organic semiconductors. 


\section{References}

[1] G. H. Gelinck, T. C. T. Geuns, and D. M. de Leeuw, Nature Mater. 3, 106 (2004)

[2] D. Voss, Nature 407, 442 (2000)

[3] G. Horowitz, J. Mater. Res. 19, 1946 (2004)

[4] C. D. Dimitrakopoulos, and P. R. L. Malenfant, Adv. Mater. 14, 99 (2002)

[5] H. Klauk, M. Halik, U. Zschieschang, G. Schmid, W. Radlik, and W. Weber, J. Appl. Phys. 92, 5259 (2002)

[6] J. Veres, S. D. Ogier, S. W. Leeming, D. C. Cupertino, and S. M. Khaffaf, Adv. Funct. Mater. 13, 199 (2003)

[7] R. W. I. de Boer, M. E. Gershenson, A. F. Morpurgo, and V. Podzorov, Phys. Stat. Sol. 201, 1302 (2004)

[8] R. W. I. de Boer, T. M. Klapwijk, and A. F. Morpurgo, Appl. Phys. Lett. 83, 4345 (2003)

[9] N. N. Iosad, G. J. Ruis, E. V. Morks, A. F. Morpurgo, N. M. van der Pers, P. F. A. Alkemade, and V. G. M. Sivel, J. Appl. Phys. 95, 8087 (2004)

[10] K. Chu, J. P. Chang, M. L. Steigerwald, R. M. Fleming, R. L. Opila, D. V. Lang, R. B. Van Dover, and C. D. W. Jones, J. Appl. Phys. 91, 308 (2002)

[11] The specific microscopic process responsible for the degradation of the organic material remains to be understood. One possible mechanism is that high-energy electrons leaking through the gate insulator physically break individual molecules at the crystal surface, thus causing the appearance of a very large number of traps in the FET active regions.

[12] A more precise quantification is difficult because an unknown fraction of leakage current flows directly to the source or drain contacts and does not cause damage to the organic crystal. 


\section{Chapter 9}

\section{Influence of the gate dielectric and charge density on the performance of rubrene single-crystal field-effect transistors}

We have performed a comparative study of rubrene single-crystal field-effect transistors fabricated using different materials as gate insulator. For all materials, highly reproducible device characteristics are obtained. The achieved reproducibility permits one to observe that the mobility of the charge carriers systematically decreases with increasing the dielectric constant of the gate insulator, the decrease being proportional to $\epsilon^{-1}$. This finding demonstrates that the mobility of carriers in organic single-crystal field-effect transistors is an intrinsic property of the crystal/dielectric interface and that it does not only depend on the specific molecule used. Additionally, single-crystal transistors with a $\mathrm{Ta}_{2} \mathrm{O}_{5}$ dielectric, in particular, exhibit an unconventional conductivity saturation, or decrease, at high gate fields, corresponding to high charge densities of $0.1-0.2$ holes/molecule. We argue that, among possible explanatory scenarios, the influence of long-range Coulomb interactions provides a physically reasonable explanation.

Parts of this Chapter have been published as A. F. Stassen, R. W. I. de Boer, N. N. Iosad, and A. F. Morpurgo, Appl. Phys. Lett. 85, 3899 (2004) 


\subsection{Introduction}

Recent research effort has led to the successful fabrication of field-effect transistors at the surface of single crystals of organic molecules. Work performed by different groups has resulted in single-crystal devices of very high quality, exhibiting an unprecedented level of reproducibility. For molecules such as tetracene, pentacene and rubrene, essentially identical results (e.g., comparable values for charge carrier mobility) have been obtained in different laboratories and using different device fabrication techniques $[1,2,3,4,5,6]$.

The quality of organic single-crystal Field-Effect Transistors (FETs) opens new opportunities for investigations of both fundamental and applied character. In particular, the use of single-crystalline devices permits to study the intrinsic - not limited by disorder - transport properties of organic semiconductors as a function of carrier density, as recently demonstrated by the observation of an anisotropic mobility in rubrene FETs exhibiting a "metallic-like" temperature dependence $[7,8]$. In addition, the reproducibility of single-crystal FETs permits to investigate in detail how different aspects of the devices influence transistor operation, which is necessary to individuate the ultimate performance limits of organic transistors. In Section 9.2, we demonstrate the influence of the dielectric constant in the gate insulator on the charge carrier mobility in the organic FET channel.

Additionally, owing to the high quality and the high reproducibility of the single-crystal FETs, new FET operation regimes become an interesting and accessible field for proceeding research. In particular, we make use of the compatibility of the electrostatic bonding technique for the fabrication of organic single-crystal FETs with the use of high- $\epsilon$ dielectrics, like $\mathrm{Ta}_{2} \mathrm{O}_{5}$, which allows to reach high charge densities. In Section 9.3 we study high charge density effects in organic semiconductors in the operation regime up to $\sim 0.2$ holes/molecule, i.e. higher densities than used in previous single-crystals OFET experiments.

\subsection{Influence of the gate dielectric on rubrene FET mobility}

In this Section, we report a comparative experimental study of the electrical characteristics of rubrene single-crystal FETs fabricated using $\mathrm{Ta}_{2} \mathrm{O}_{5}, \mathrm{Al}_{2} \mathrm{O}_{3}, \mathrm{SiO}_{2}$, and Parylene $\mathrm{C}$ as gate insulator. For the different dielectrics, field-effect transistors exhibiting stable and hysteresis-free electrical behavior can be reproducibly realized. In all cases, the hole mobility extracted from room-temperature mea- 


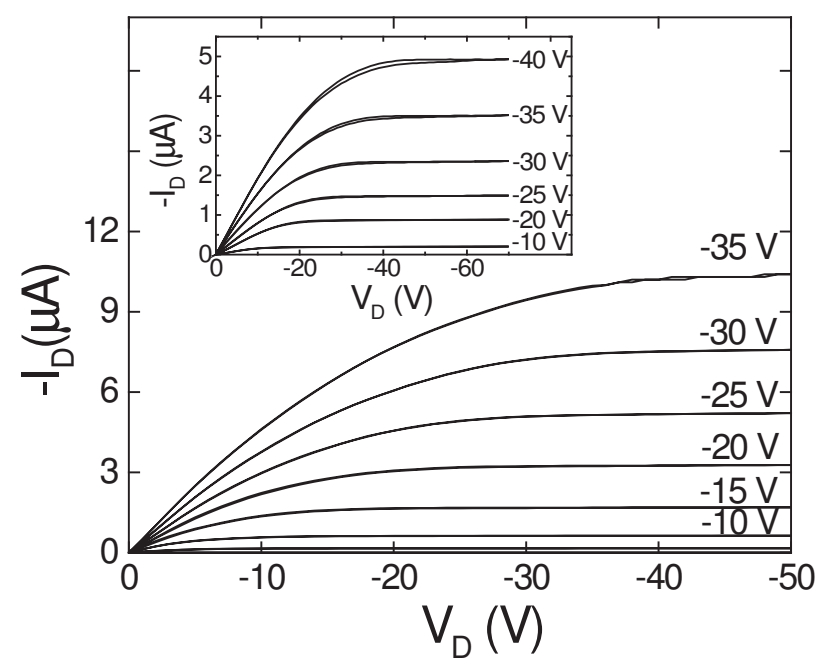

Figure 9.1: Source-drain current versus source-drain voltage measured at different gate voltages for a device fabricated on $\mathrm{SiO}_{2}$, with an $L=1.2 \mathrm{~mm}$ channel length and $W=200 \mu \mathrm{m}$ channel width. The inset shows similar data for a FET fabricated using parylene $\mathrm{C}(L=650 \mu \mathrm{m}, W=340 \mu \mathrm{m})$.

surement of the transistor characteristics is remarkably gate-voltage independent. From these measurements, we find that the mobility $\mu$ decreases from $10 \mathrm{~cm}^{2} / \mathrm{Vs}$ (Parylene $\mathrm{C}, \epsilon=3.15)$ to $1.5 \mathrm{~cm}^{2} / \mathrm{Vs}\left(\mathrm{Ta}_{2} \mathrm{O}_{5}, \epsilon=25\right)$ with increasing the relative dielectric constant $\epsilon$. By comparing our data to those recently reported for transistors fabricated using Parylene $\mathrm{N}\left(\mu=15 \mathrm{~cm}^{2} / \mathrm{Vs} ; \epsilon=2.65\right)[2,9]$ and polydimethylsiloxane (PDMS) air-gap stamps $\left(\mu=20 \mathrm{~cm}^{2} / \mathrm{Vs} ; \epsilon=1\right)[7]$, we conclude that a decrease in mobility with increasing the dielectric constant of the gate dielectric occurs systematically in rubrene single-crystal FETs. This result demonstrates that the mobility measured in organic transistors is not only a property of the specific organic molecule used, but that it intrinsically depends on the organic/dielectric interface.

\subsubsection{Technical aspects}

The devices used in our investigations have been fabricated by means of two different, recently developed techniques. Transistors based on Parylene $\mathrm{C}$ have been built following the processing described in Section 3.2.2, using aqueous colloidal graphite or silver epoxy for the source, drain, and gate electrodes (with colloidal graphite resulting in better performances as compared to epoxy). For these devices, rather thick crystals (typically $100 \mu \mathrm{m}$ or thicker) were used. All other transistors were fabricated by means of electrostatic bonding of much thinner 
(approximately $1 \mu \mathrm{m}$ thick) crystals on doped Si substrates with pre-fabricated FET circuitry, using a process identical to the one described in Ref. [3]. In all cases, the crystals were grown in a horizontal oven similar to the system used in Ref. [10] with argon as carrier gas $(50 \mathrm{ml} / \mathrm{min})$. The majority of the rubrene crystals used in our investigations were needle shaped. As discussed in Ref. [7], these needle-shaped crystals grow preferentially along the crystallographic $b$-axis, which corresponds to the direction of highest hole mobility. Both the parylene gate and the oxide-gate transistors were fabricated with a large source-drain distance (always larger than $300 \mu \mathrm{m}$ and typically $\sim 1 \mathrm{~mm}$ ) to ensure that the measured transport occurred preferentially along this direction and to minimize contact effects on measurements performed in a two-terminal configuration.

In the case of $\mathrm{SiO}_{2}$-based transistors, the gate insulator was a $200 \mathrm{~nm}$ thick, thermally grown oxide layer. In the case of $\mathrm{Ta}_{2} \mathrm{O}_{5}$, a $\mathrm{Nb}$ layer acting as a gate was sputter-deposited onto the Si substrate, followed by a $375 \mathrm{~nm}$ thick layer of $\mathrm{Ta}_{2} \mathrm{O}_{5}$. Sputtering of $\mathrm{Ta}_{2} \mathrm{O}_{5}$ was performed from a metallic Ta target in the presence of oxygen in an argon plasma, with the substrate held at approximately $300^{\circ} \mathrm{C}$, according to the procedure developed in Ref. [11] (and resulting in the "type II" $\mathrm{Ta}_{2} \mathrm{O}_{5}$ investigated in Chapter 8). Finally, $\mathrm{Al}_{2} \mathrm{O}_{3}$ devices were fabricated by sputtering a $25 \mathrm{~nm}$ layer of $\mathrm{Al}_{2} \mathrm{O}_{3}$ on top of a $\mathrm{Ta}_{2} \mathrm{O}_{5}$ layer. The roughness of the three different oxide layers was measured using an atomic force microscope and found to have a root mean square value less than $0.1 \mathrm{~nm}$. Fig. 3.6 shows an optical image of a rubrene single-crystal FET fabricated by means of electrostatic bonding on $\mathrm{SiO}_{2}$.

\subsubsection{Experimentally observed influence of gate dielectric}

Fig. 9.1 shows typical source-drain voltage-current $I_{D}$ versus $V_{D}$ sweeps taken at different values of the voltage applied to the gate electrode $\left(V_{G}\right)$ for a singlecrystal FET on $\mathrm{SiO}_{2}$ (the inset shows similar data for a transistor fabricated using Parylene C). All measurements discussed here were performed in a two-terminal configuration in vacuum ( $10^{-6}$ mbar), at room temperature, using a HP4156A semiconductor parameter analyzer. As shown in Fig. 9.1, essentially no hysteresis is present in the measurements and the $I_{D}$ versus $V_{D}$ are linear down to small applied $V_{D}$ voltages. For electrostatically bonded transistors we performed in a number of cases four-terminal measurements and observed that owing to large length of the FET channel the contact resistance does not significantly affect the value of mobility observed in a two terminal configuration. In the case of Parylene C, the manually deposited contacts are often of lower quality and the application of a large source-drain bias is required to avoid contact limitations to 
(a)

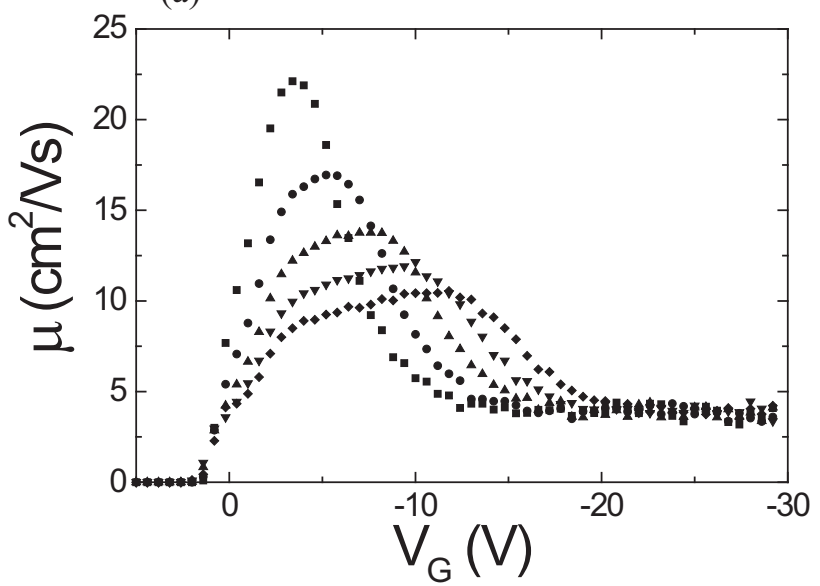

(b)

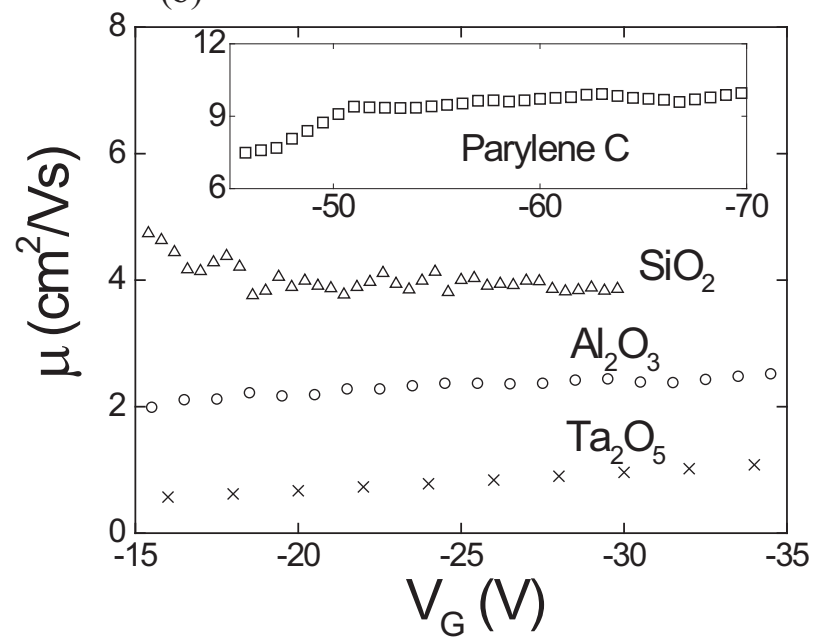

Figure 9.2: (a) Mobility versus gate voltage for a device on $\mathrm{SiO}_{2}$, measured at different values of source-drain voltage $\left(V_{D}=-5,-7.5,-10,-12.5,-15 \mathrm{~V}\right.$, respectively), obtained using Eq. 9.1. Note that in the linear regime $\left(V_{G} \gg V_{D}\right) \mu$ does not depend on $V_{G}$ and $V_{D}$ (the apparent peak at low $V_{G}$ values is an artifact originating from the use of Eq. 9.1 outside the linear regime). (b) $\mu\left(V_{G}\right)$ curves as measured for the four different gate insulators. For device based on parylene $\mathrm{C}$, the suppression of contact effects often requires the use of a rather large value $V_{D}$ (and thus $V_{G}$, to remain in the linear regime).

the current flowing through the devices.

We use the relation ( $W$ is the channel width, $L$ the channel length, and $C_{i}$ the capacitance per unit area)

$$
\mu=\frac{L}{W} \cdot \frac{1}{C_{i}} \cdot \frac{1}{V_{D}} \cdot \frac{\delta I_{D}}{\delta V_{G}}
$$


to extract the value of the mobility from the linear part of the transistor characteristics, as a function of gate voltage. Fig. 9.2a shows the mobility data obtained using this relation for different values of the source drain voltage $\left(V_{D}=-5,-7.5\right.$, $-10,-12.5$, and $-15 \mathrm{~V}$ respectively) for one of the electrostatically bonded transistors with $\mathrm{SiO}_{2}$ gate insulator. The same value of mobility is obtained independent of $V_{G}$ and $V_{D}$ in all cases, as long as the device operates in the linear regime for which Eq. 9.1 applies, i.e. if $V_{G}$ is sufficiently larger than $V_{D}$ (the apparent peak in mobility present at low $V_{G}$ is an artifact due to the violation of this condition). The field-effect mobility extracted from the transistor $I-V$ characteristics as a function of the applied gate voltage is shown in Fig. 9.2b for transistors fabricated using all the different gate insulators.

Fig. 9.2b also shows that the mobility of rubrene single-crystal FETs is different for devices fabricated with the different gate dielectrics. Although all transistors fabricated show a spread in the values of measured mobilities, probably originating from defects induced by the crystal handling during the fabrication process, we found that a large fraction of devices exhibit mobilities in a rather narrow range of values. Specifically, for transistors fabricated using Parylene $\mathrm{C}$ $\mu$ typically ranges between 6 and $10 \mathrm{~cm}^{2} / \mathrm{Vs}$, for $\mathrm{SiO}_{2}$ between 4 and $6 \mathrm{~cm}^{2} / \mathrm{Vs}$, for $\mathrm{Al}_{2} \mathrm{O}_{3}$ devices best $\mu$ values are $2-3 \mathrm{~cm}^{2} / \mathrm{Vs}$, and for $\mathrm{Ta}_{2} \mathrm{O}_{5} 1-1.5 \mathrm{~cm}^{2} / \mathrm{Vs}$. From these data, it is apparent that for all investigated rubrene FETs the measured mobility systematically decreases when increasing the dielectric constant of the gate insulator. This trend is consistent with the results obtained by others on rubrene FETs fabricated using Parylene N $(\epsilon=2.65)$, for which the measure mobility ranges between 10 and $15 \mathrm{~cm}^{2} / \mathrm{Vs}$ [9], and vacuum $(\epsilon=1)$ where the mobility range is $16-20 \mathrm{~cm}^{2} / \mathrm{Vs}[7]$.

Fig. 9.3 summarizes the available data and clearly demonstrates the dependence of $\mu$ on the dielectric constant $\epsilon$. The inset of Fig. 9.3 additionally shows that, when plotted on a log-log scale, the mobility decrease with increasing $\epsilon$ as $\epsilon^{-1}$ over the entire range available (slightly more than one decade). From these data we directly conclude that the mobility of organic FETs cannot be simply considered to be an intrinsic property of the molecular material used but rather that it is intrinsically a property of the organic/dielectric interface. This conclusion is also supported by our measurements on tetracene single-crystal FETs, in which we have observed that the mobility of $\mathrm{SiO}_{2}$ based devices is systematically larger than that of transistors fabricated using $\mathrm{Ta}_{2} \mathrm{O}_{5}$ as a gate insulator. 


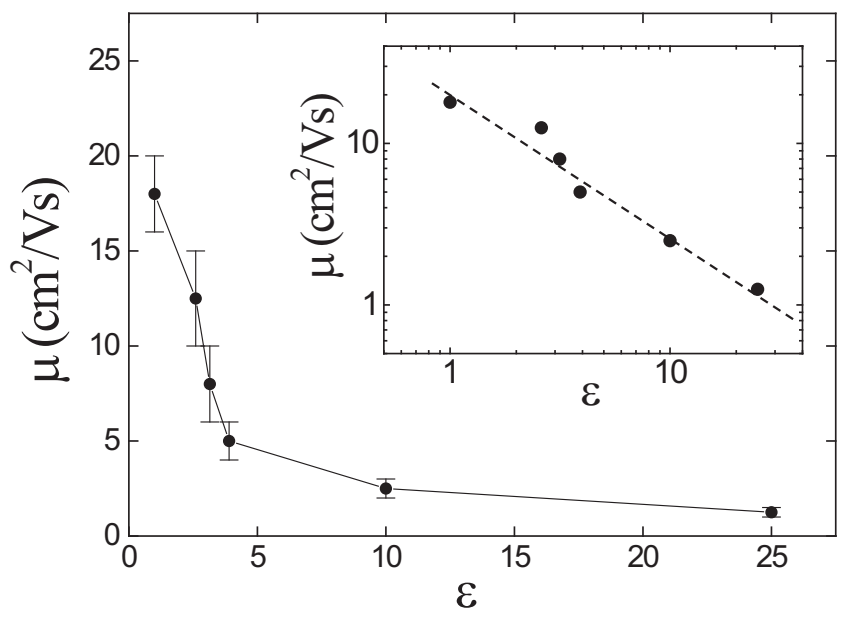

Figure 9.3: Decrease of the mobility with increasing $\epsilon$, as observed in rubrene singlecrystal FETs with different gate insulators. The bars give a measure of the spread in mobility values. Inset: when plotted on a log-log-scale, the available data show a linear dependence with slope -1 (i.e. the variation in $\mu$ is proportional to $\epsilon^{-1}$ ).

\subsubsection{Proposed mechanism of polaronic dressing}

A systematic decrease in $\mu$ with increasing $\epsilon$ has recently been reported for disordered polymeric organic FETs of lower mobility [12]. In that context, the effect has been attributed to the localized nature of the charge carriers in the material and their interaction with the induced polarization in the gate insulator. Specifically, according to the authors of Ref. [12], dipolar disorder in the dielectric, which is stronger the larger the value of the dielectric constant, induces a broadening of the density of states (DOS) at the polymer/insulator interface. This broadening results in a decrease of the DOS at the Fermi energy, which, in a disordered material, causes a lower hopping probability. This lower hopping probability leads to a suppression of the carrier mobility, in agreement with theoretical work by Bässler [13].

In single-crystalline devices, disorder is much weaker than in polymers. Nevertheless, even in the best rubrene single-crystal FETs at room temperature holes are nearly localized by polaronic effects and cannot be described in terms of extended states, as it is the case for conventional inorganic semiconductors. Therefore, also in single-crystal FETs, a (nearly) localized charge carrier at the rubrene/dielectric interface locally polarizes the dielectric. The electrostatic potential generated by the induced polarization exerts an attractive force on the charge carrier itself that increases the tendency towards carrier self-trapping. As 
the attractive force is larger for larger $\epsilon$, this qualitatively explains why the mobility is reduced with increasing $\epsilon$. Stated differently, at the interface between the crystal and the gate insulator, the electrical polarizability of the environment experienced by the charge carriers is determined by the dielectric constant of the insulating material. In this way, the polaronic dressing of charge carriers is enhanced by the presence of a gate insulator with a large dielectric constant. As a consequence of this enhanced polaronic dressing, the mobility is reduced.

The detailed microscopic understanding of the mechanism just proposed clearly requires (and deserves) further experimental and theoretical investigations. From a fundamental perspective, this mechanism is interesting, since it permits to tune polaronic effects in a FET configuration, thus offering a new tool for their study. For instance, experimentally, it will be interesting to look in detail at how the temperature dependence of the mobility evolves from the metallic-like regime $(d \mu / d T<0)[8]$ to the thermally activated regime $(d \mu / d T>0)[14]$ with increasing $\epsilon$ (work is in progress in this direction). Finally, our findings are also relevant for applications, as they clearly demonstrate that the speed of organic transistors can be enhanced by using low- $\epsilon$ gate insulators.

\subsection{High charge density study on rubrene FETs}

The compatibility of the electrostatically bonded FET technique with high- $\epsilon$ dielectrics, in combination with the high quality of the organic single-crystal FETs, allows to investigate high charge density effects in organic semiconductors. Specific interest in this operation regime originates from the expected strong Coulomb interaction between charge carriers in organic molecular crystals, that stems from the narrow bandwidth. Indeed, it is well-known that at a sufficiently high density of chemically-induced carriers, the potassium-doped fullerene $\mathrm{K}_{\mathrm{x}} \mathrm{C}_{60}$ exhibits superconductivity $(\mathrm{x}=3)$ and a Mott-Hubbard insulating state $(\mathrm{x}=4)[15,16]$. Moreover, first-principles calculations on oligo-acenes and -thiophenes have shown that the on-site Coulomb repulsion between charge carriers on one molecule is larger than the electronic bandwidth [17], which is relevant for charge densities close to half filling, i.e. $\sim 0.5$ carriers per molecule.

In this Section we investigate charge transport in rubrene at charge densities up to $\sim 0.2$ holes per molecule, by using a high- $\epsilon \mathrm{Ta}_{2} \mathrm{O}_{5}$ gate dielectric. Whereas at low gate fields, the electrical FET characteristics show the conventional highquality single-crystal OFET behavior, we observe an unexpected reversible saturation, or decrease, of the conductivity at high gate fields, i.e. for $0.1-0.2$ holes per molecule. A comparison with other organic FET measurements is in- 


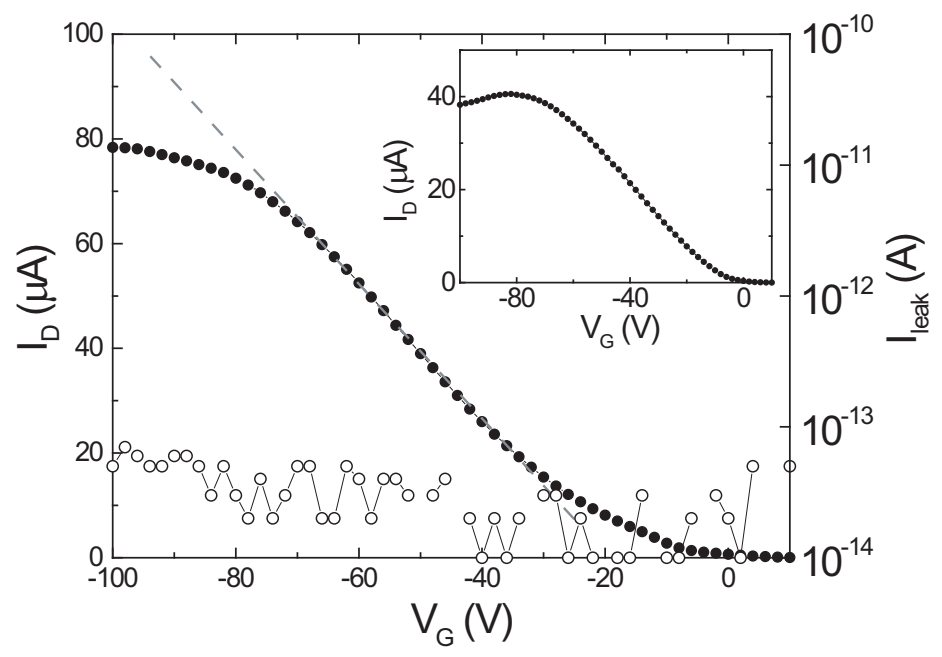

Figure 9.4: $I_{D}-V_{G}$ curve (closed circles) of a rubrene FET on $\mathrm{Ta}_{2} \mathrm{O}_{5}\left(V_{D}=-10 \mathrm{~V}\right)$. For gate voltages $-100 \mathrm{~V}<V_{G}<-70 \mathrm{~V}$, the drain current exhibits an unconventional saturation, implying that the conductivity saturates for increasing $V_{G}$. The open symbols represent the leakage current $I_{\text {leak }}$, which is $<10^{-9} \mathrm{~A} / \mathrm{cm}^{2}$ over the whole measured range. The inset shows the $I_{D}-V_{G}$ curve of a rubrene FET on $\mathrm{Ta}_{2} \mathrm{O}_{5}$ that exhibits an unconventional conductivity decrease when approaching $V_{G}=-100 \mathrm{~V}$.

sufficient to explain the distinct behavior in this, so far, unexplored operation regime. Using simple argumentations and first approximations, we will discuss scenarios that provide a possible explanation for the conductivity saturation or decrease.

\subsubsection{Unconventional FET characteristics}

The fabrication of the rubrene FETs with $\mathrm{Ta}_{2} \mathrm{O}_{5}$ dielectric is described in detail in the previous Section. We use the "type II" $\mathrm{Ta}_{2} \mathrm{O}_{5}$, discussed in Chapter 8, with breakdown field $E_{\mathrm{bd}} \simeq 4-6 \mathrm{MV} / \mathrm{cm}$ and leakage currents $<10^{-9} \mathrm{~A} / \mathrm{cm}^{2}$ at half the breakdown field. Our measurements span the range from 0 to $4 \cdot 10^{13}$ holes $/ \mathrm{cm}^{2}$, corresponding to charge densities up to $\sim 0.2$ carriers per molecule. This density is $\sim 4$ times higher than the charge densities that are reached when using a $\mathrm{SiO}_{2}$ dielectric.

The rubrene FETs exhibit unconventional behavior at high gate fields. Figure 9.4 shows a typical source-drain current $I_{D}$ versus gate voltage $V_{G}$ sweep taken at fixed source-drain voltage $V_{D}$. At voltages $V_{G}=-35$ to $-70 \mathrm{~V}$, the $I_{D}-V_{G}$ relation in Fig. 9.4 is linear, as expected for standard FET behavior with $V_{G}>V_{D}$ [18]. However, at gate voltages larger than $-70 \mathrm{~V}$, there is a clear 


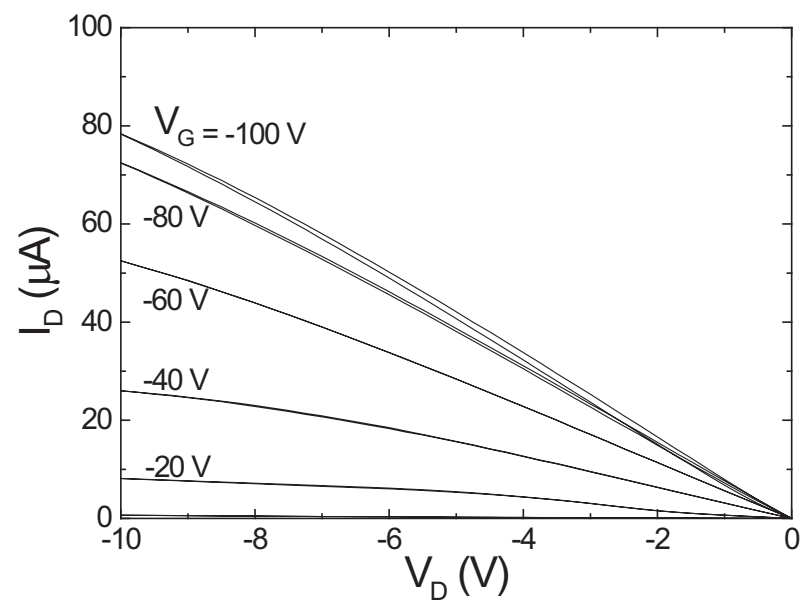

Figure 9.5: Current-voltage characteristics of an organic single-crystal FET, with rubrene as the organic molecule and $\mathrm{Ta}_{2} \mathrm{O}_{5}$ as the gate insulator.

deviation from the linear behavior. The $I_{D}-V_{G}$ curve saturates, indicating a saturation of the FET channel conductivity. Contrary to the degradation discussed in Chapter 8, this behavior is reversible: repeating the $I_{D}-V_{G}$ sweep results in the same curve, with the non-linearity starting at the same value of $V_{G}$. The reversible saturation in conductivity is accompanied by small hysteresis in the $I_{D}-V_{D}$ sweeps, as visible in Fig. 9.5 for the same rubrene FET on $\mathrm{Ta}_{2} \mathrm{O}_{5}$.

The distinctness of the non-linearity at high gate voltages is emphasized by the standard behavior that is observed at lower $V_{G}$. To accentuate the linear regime of the $I_{D}-V_{G}$ behavior (from -35 to $-70 \mathrm{~V}$ ), a line is drawn in Fig. 9.4. Using Eq. 9.1 to extract the value of the mobility from the linear part of Fig. 9.4 gives $\mu=0.3 \mathrm{~cm}^{2} / \mathrm{Vs}$. This mobility is in the range of values commonly found for rubrene on $\mathrm{Ta}_{2} \mathrm{O}_{5}$ [19]. Also the $I_{D}-V_{D}$ sweeps for different $V_{G}$ values in this voltage regime show a behavior that is comparable to previously measured high-quality organic single-crystal FETs, as shown in Fig. 9.5: the $I_{D}-V_{D}$ curves are linear down to small applied $V_{D}$, and essentially no hysteresis is present in the measurements for $V_{G} \leq-60 \mathrm{~V}$.

The observations indicate that the conductivity saturation is caused by a process that is influential at high gate fields, but insignificant at low fields. This is emphasizes by the measurements shown in the inset of Fig. 9.4, where the conductivity does not saturate at increasing gate voltage, but actually decreases when approaching $V_{G}=-100 \mathrm{~V}$. We have investigated two rubrene FETs on type II $\mathrm{Ta}_{2} \mathrm{O}_{5}$, which both show the non-linear behavior at high $V_{G}$. The value at which the non-linearity starts (around $-70 \mathrm{~V}$ for the device in Fig. 9.4) differs 
by less than $10 \mathrm{~V}$ in the two devices. In Fig. 9.4, the rate of sweeping $V_{G}$ during the measurement is $\sim 0.05 \mathrm{~V} / \mathrm{s}$. The conductivity saturation is less pronounced for measurements with a faster sweep rate and can even disappear completely. In Chapter 8, the lower panel panel of Fig. 8.3 illustrates an $I_{D}-V_{G}$ plot in which no significant conductivity saturation is observed. These data were taken on the same rubrene FET as the results shown in Fig. 9.4, but with a sweep rate of $1 \mathrm{~V} / \mathrm{s}$.

\subsubsection{Discussion of explanatory scenarios}

The data we have obtained so far do not provide enough information to determine the origin of the conductivity saturation or decrease at high gate fields. Nevertheless, we can think of several scenarios that are expected to result in such behavior. For example, a decreasing conductivity is expected when approaching complete filling of an energy band [20,21]. This effect is expected to influence charge transport when achieving $0.5-1$ charge carriers per molecule, and since our experiments do not reach that regime, we believe that simple band filling is an unlikely explanation. Also a sharp maximum in the density of states (DOS) as a function of energy does not seem a likely candidate to explain the data, since in first approximation, the DOS is expected to be featureless owing to smoothing by (intrinsic or extrinsic) disorder. We can think of two other possible scenarios, namely long-range Coulomb interactions between holes, and increased trapping of carriers by high applied electrostatic gate fields. In the following, we will go deeper into the two scenarios and discuss the physical consequences of these phenomena in organic FETs. We will argue that, although we cannot exclude the influence of trapping, the effect of Coulomb interactions provides a physically reasonable explanation for the observed reversible conductivity saturation and decrease.

The charge densities reached in our measurements are below the regime where the on-site Coulomb interactions [17], that we mention in the introduction of this Section, influence the conductivity. However, the long-range Coulomb repulsion between charge carriers in rubrene is significant already at lower charge densities. The general equation to calculate the Coulomb energy between two charges $e$ at a distance $r$ is the following:

$$
E_{C}=\frac{e^{2}}{4 \pi \epsilon \epsilon_{0} r}
$$

where $\epsilon$ is the relative dielectric constant of the material in which the charges reside, and $\epsilon_{0}$ is the dielectric constant of vacuum. A hole density of $\sim 0.2$ holes 
per molecule corresponding to an average distance of roughly $2 \mathrm{~nm}$ between the holes. Using Eq. 9.2 as a first approximation, the Coulomb interaction energy can be estimated to be $\sim 0.3 \mathrm{eV}$. In general, to determine the significance of electronelectron interaction effects in a solid, the Coulomb energy is typically compared to the kinetic energy of the charge carriers. However, although calculations give for bulk organic materials bandwidths of $W \leq 0.5 \mathrm{eV}[17,22]$, the electrical transport in our rubrene FETs on $\mathrm{Ta}_{2} \mathrm{O}_{5}$ exhibits a thermally excited temperature dependence, implying that kinetic energy of charge carriers in these devices is much smaller than the calculated bandwidths. Instead, the thermal energy is dominant, and therefore, we compare the value of $E_{C}$ to $k_{B} T$. For increasing charge density, $E_{C}$ grows and becomes larger than $k_{B} T$. We conclude that at a density of $\sim 0.2$ holes per molecule the Coulomb repulsive energy $E_{C} \simeq 0.3 \mathrm{eV}$ is significantly larger than $k_{B} T$ at room temperature.

The charge density dependence of the Coulomb interaction is complicated due to screening of the Coulomb potential by the highly-polarizable $\mathrm{Ta}_{2} \mathrm{O}_{5}$ dielectric. In Eq. 9.2, the dielectric constant $\epsilon$ accounts for electrostatic screening of the Coulomb potential by the organic material surrounding the charge. The high polarizability of the high- $\epsilon \mathrm{Ta}_{2} \mathrm{O}_{5}$ leads to additional screening of the Coulomb potential for a charge carrier at the organic/dielectric interface [23]. The screened Coulomb field can be compared to the summed field of an excess charge on a rubrene molecule and its induced image charge in the dielectric (see Fig. 9.6). The distance between the charge and its image charge roughly equals the rubrene interlayer distance, i.e. $\sim 3 \mathrm{~nm}$, which indicates that the screening is mainly efficient at distances $\gtrsim 3 \mathrm{~nm}$ from the excess charge. Thus, the influence of the highly-polarizable $\mathrm{Ta}_{2} \mathrm{O}_{5}$ reduces the effects of Coulomb interactions for carrier distances larger than $\sim 3 \mathrm{~nm}$, corresponding to densities $<0.1$ holes/molecule, but it does not severely affect the Coulomb repulsion at higher charge densities.

We conclude that simple argumentation suggests significant electron-electron interactions at the charge densities reached in our experiments. In general, significant electron-electron interactions have consequences for both the charge carrier mobility and the capacitance of a FET. Electron-electron interactions are envisioned to reduce the charge carrier mobility due to localization of the conduction carriers by means of charge ordering. Several existing models describe localization effects by charge ordering, like the theory of Wigner solids [24], and models that are committed to charge ordering phenomena in organic charge transfer salts with quarter-filled bands $[25,26,27]$. It goes beyond the scope of this study, though, to discuss whether one of these models provides an appropriate description of the effects discussed in this Section. In addition, electron-electron interactions are also foreseen to reduce the FET capacitance. In case of non-zero electron-electron 


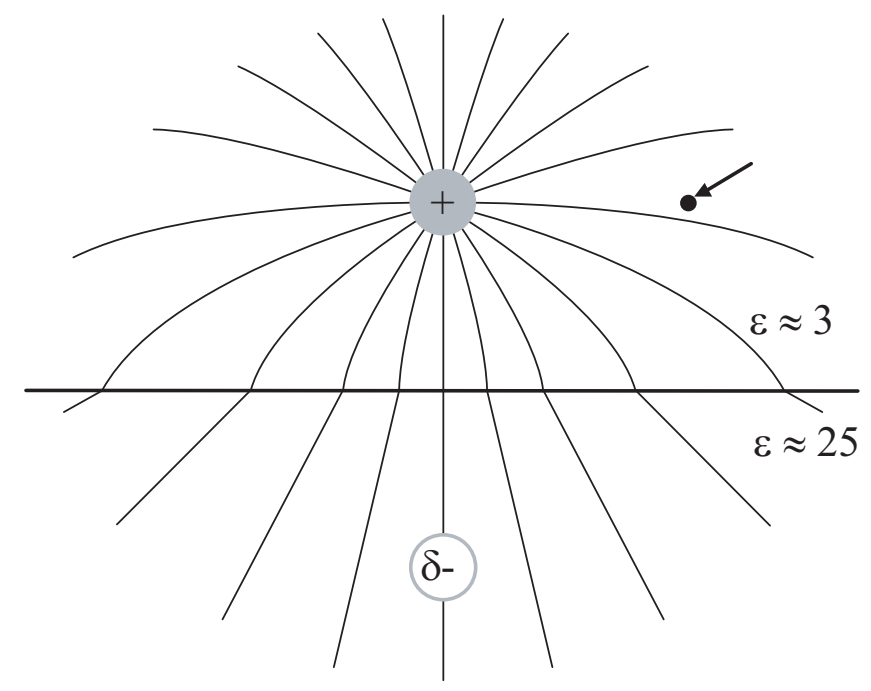

Figure 9.6: Schematic drawing of electric field lines around a charge on an organic molecule, close to the interface of rubrene $(\epsilon=3)$ and $\mathrm{Ta}_{2} \mathrm{O}_{5}$ dielectric $(\epsilon=25)$ [23]. For simplicity, the two materials are considered as homogenous media. The distance between the positive charge $(+)$ and its induced image charge $(\delta-)$ in the dielectric is $\sim 3 \mathrm{~nm}$. The arrow indicates a position that is at $\sim 2 \mathrm{~nm}$ distance from the positive charge, where the Coulomb field of the charge is not severely affected by screening due to the highly-polarizable dielectric.

interactions, the addition of a carrier requires, besides the "standard" charging energy of a capacitor, an extra charging energy that results from the strong Coulomb repulsion of the added carrier with all its surrounding carriers. Consequently, the capacitance of the FET decreases with increasing charge density, due to the growing Coulomb repulsion [28]. Both a reduction in the mobility and a lower FET capacitance contribute to a saturating, or even decreasing, conductivity for increasing electron-electron interactions. The experimentally observed deviation of the FET source-drain current from the anticipated linear relation with increasing $V_{G}$ is in agreement with this prediction.

An alternative explanation for the conductivity saturation (decrease) is provided by increased trapping of charge carriers due to high applied electrostatic gate fields. Traps are known to have a significant limiting effect on the experimental charge carrier mobility. Moreover, hysteresis in the FET characteristics, as observed in Fig. 9.5 for $V_{G}<-60 \mathrm{~V}$, is generally interpreted as due to traps present at the organic/dielectric interface [29, 30, 31]. An interpretation of the distinct high- $V_{G}$ behavior of rubrene FETs in terms of trapping is provided by the appearance of a growing amount of hole trapping states at increasing gate field, resulting in a lower number of mobile holes that contribute to the charge 


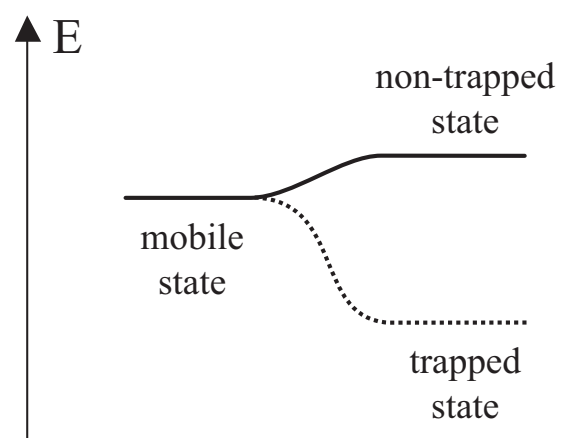

Figure 9.7: An energy diagram illustrating an example of a gate-field sensitive, reversible trapping mechanism. The "mobile state" indicates the energy level of a mobile charge carrier. At the right of it, a gate-field dependent energy level is sketched: at low gate field the level is higher in energy than the mobile state and thus ineffective as a trap ("non-trapped state"), and at high gate field the level shift below the mobile state and is an active trap ("trapped state"). When consecutively reducing the gate field again, the energy level shifts back to the non-trapped state.

transport.

The reproducibility of our measurements indicates that the additional traps at high $V_{G}$ are not permanently induced. In particular, a shift in the threshold voltage, that is a typical sign for the creation of deep traps, is absent in consecutive $I_{D}-V_{G}$ sweeps. This indicates that the unconventional conductivity change involves electronic states that are reproducibly ineffective as traps at low gate fields, and active hole traps at higher fields, requiring a gate-field sensitive and reversible trapping mechanism. For instance, a possible mechanism is provided by states that are situated around or above the energy of mobile holes, where they are ineffective as traps, and shift into the bandgap under the influence of a high gate field, where they become effective carrier traps (see Fig. 9.7).

We are not aware, though, of any publications that report on the existence of such gate-field-sensitive trapping mechanisms. Consequently, the requirement of such unconventional trapping concepts reduces the credibility of the trapping scenario. As opposed to that, the influence of Coulomb repulsion is in first approximation, and using a minimum of assumptions, expected to play a significant role in our measurements. We conclude that, although we cannot exclude the influence of trapping, the effect of Coulomb interactions provides a physically reasonable explanation for the observed reversible conductivity saturation and decrease.

In conclusion, we have experimentally explored charge transport properties of rubrene single-crystal FETs with $\mathrm{Ta}_{2} \mathrm{O}_{5}$ dielectric at charge densities up to 
0.2 holes per molecule and we observe a reversible conductivity decrease close to the highest applied gate fields. We have discussed possible scenarios explaining our results, of which the influence of long-range Coulomb interactions provides a physically reasonable explanation, due to its simplicity of arguments.

\section{References}

[1] V. Podzorov, V. M. Pudalov, and M. E. Gershenson, Appl. Phys. Lett. 82, 1739 (2003)

[2] V. Podzorov, S. E. Sysoev, E. Loginova, V. M. Pudalov, and M. E. Gershenson, Appl. Phys. Lett. 83, 3504 (2003)

[3] R. W. I. de Boer, T. M. Klapwijk, and A. F. Morpurgo, Appl. Phys. Lett. 83, $4345(2003)$

[4] V. Y. Butko, X. Chi, D. V. Lang, and A. P. Ramirez, Appl. Phys. Lett. 83, $4773(2003)$

[5] J. Takeya, C. Goldmann, S. Haas, K. P. Pernstich, B. Ketterer, and B. Batlogg, J. Appl. Phys. 94, 5800 (2003)

[6] R. W. I. de Boer, M. E. Gershenson, A. F. Morpurgo, and V. Podzorov, Phys. Stat. Sol. A 201, 1302 (2004)

[7] V. C. Sundar, J. Zaumseil, V. Podzorov, E. Menard, R. L. Willett, T. Someya, M. E. Gershenson, and J. A. Rogers, Science 303, 1644 (2004)

[8] V. Podzorov, E. Menard, A. Borissov, V. Kiryukhin, J. A. Rogers, and M. E. Gershenson, Phys. Rev. Lett. 93, 086602 (2004)

[9] M. E. Gershenson (private communication)

[10] R. A. Laudise, C. Kloc, P. G. Simpkins, and T. Siegrist, J. Cryst. Growth 187, 449 (1998)

[11] N. N. Iosad, G. J. Ruis, E. V. Morks, A. F. Morpurgo, N. M. van der Pers, P. F. A. Alkemade, and V. G. M. Sivel, J. Appl. Phys. 95, 8087 (2004)

[12] J. Veres, S. D. Ogier, S. W. Leeming, D. C. Cupertino and S. D. Khaffaf, Adv. Funct. Mater. 13, 199 (2003)

[13] H. Bässler, Philos. Mag. 50, 347 (1984)

[14] R. W. I. de Boer (unpublished)

[15] A. F. Hebard, M. J. Rosseinsky, R. C. Haddon, D. W. Murphy, S. H. Glarum, T. T. M. Palstra, A. P. Ramirez, and A. R. Kortan, Nature 350, 600 (1991) 
[16] O. Gunnarsson, E. Koch, and R. M. Martin, Phys. Rev. B 54, 11026 (1996)

[17] G. Brocks, J. van den Brink, and A. F. Morpurgo, Phys. Rev. Lett. 93, 146405 (2004)

[18] S. M. Sze, Physics of Semiconductor Devices (Wiley, New York, 1981)

[19] See Chapter 8 and Section 9.2

[20] R. C. Haddon, A. F. Hebard, M. J. Rosseinsky, D. W. Murphy, S. J. Duclos, K. B. Lyons, B. Miller, J. M. Rosamilia, R. M. Fleming, A. R. Kortan, S. H. Glarum, A. V. Makhija, A. J. Muller, R. H. Eick, S. M. Zahurak, R. Tycko, G. Dabbagh, and F. A. Thiel, Nature 350, 320 (1991)

[21] M. F. Craciun, S. Rogge, M. J. L. den Boer, T. M. Klapwijk, and A. F. Morpurgo, J. Phys. IV 114, 607 (2004)

[22] M. Pope, and C. E. Swenberg, Electronic Processes in Organic Crystals and Polymers, 2nd ed. (Oxford University Press, New York, 1999)

[23] W. D. Jackson, Classical Electrodynamics, 2nd ed., Chapter 4 (Wiley, New York, 1975)

[24] E. Wigner, Phys. Rev. 46, 1002 (1934)

[25] T. Ishiguro, K. Yamaji, and G. Saito, Organic Superconductors (Springer, Berlin, 1998)

[26] R. T. Clay, S. Mazumdar, and D. K. Campbell, Phys. Rev. B 67, 115121 (2003)

[27] M. Dressel, and N. Drichko, Chem. Rev. 104, 5689 (2004)

[28] Due to Coulomb repulsion between the carriers, the more states are filled, the further the unfilled states are pushed upward in energy. In other words, the Coulomb repulsion also affects the density of states (DOS) in the semiconductor as a function of energy, causing a lower DOS around the Fermi level at higher energy. Also this effect can contribute to a lower capacitance at increasing gate field.

[29] M. Matters, D. M. de Leeuw, P. T. Herwig, and A. R. Brown, Synth. Met. 102, 998 (1999)

[30] D. Knipp, R. A. Street, A. Völker, and J. Ho, J. Appl. Phys. 93, 347 (2003)

[31] M. J. Panzer, C. R. Newman, and C. D. Frisbie, Appl. Phys. Lett. 86, 103503 (2005) 


\section{Summary}

\section{Organic single-crystal field-effect transistors Ruth W. I. de Boer}

Organic electronics constitute an innovative field, with interesting applications complementary to the silicon semiconductor technology. From a scientific perspective, there is large interest in the fundamental understanding of electrical transport in organic semiconductors. However, a well-developed microscopic description is still lacking, due to the complicated character of the many-body polaronic-type of charge carriers in molecular compounds.

In this Thesis, we have experimentally studied the intrinsic charge transport properties of organic semiconductors by using organic single-crystal field-effect transistors. The electric field-effect has been frequently used to investigate thin films of organic compounds. Unfortunately, thin-film transistors are not suitable for the study of intrinsic electronic properties of organic conductors, because their characteristics are often strongly affected by imperfections of the film structure and by insufficient purity of organic materials. Thus, for a higher degree of molecular ordering and an improved quality of the FET, we fabricate devices on the surface of a free-standing single crystal of organic molecules.

Starting our work with the characterization of as-grown single crystals, we have systematically studied electrical transport in single crystals of tetracene by means of Space Charge Limited Current (SCLC) spectroscopy and Time of Flight (TOF) measurements. Both types of DC transport measurements show that the room-temperature effective hole-mobility in tetracene reaches values close to $\mu \simeq 1 \mathrm{~cm}^{2} / \mathrm{Vs}$ and show that, within a range of temperatures, the mobility increases with decreasing temperature. The latter observation is generally considered as a signature of the intrinsic electrical properties and its perceptibility in the transport measurements demonstrates the high quality of the crystals.

In a more detailed study of SCLC measurements and theory, we have show that deep traps at the surface of a semiconducting material, underneath the electrical contacts used to inject charge carriers, are an essential ingredient for the proper understanding of SCLC $I-V$ curves, which has been neglected until now. 
The main effect of charge trapped at the surface is to modify the electrostatic profile throughout the entire bulk of the sample, profoundly affecting the behavior of SCLC. By simulating SCLC $I-V$ curves and taking into account the presence of these traps, we can reproduce experimental observations not captured by the conventional theory that only considers the presence of traps in the bulk of the material.

The main source for the surface traps in organic single-crystal devices is damage that is induced during the deposition of electrical contacts, which indicates that material deposition on the surface of an organic crystal is a delicate process. This engenders a considerable complication to the fabrication of high-quality field-effect transistors on organic single crystals. Thus, an important achievement described in this thesis is the development of the electrostatic bonding technique for processing organic single-crystal FETs.

The electrostatic bonding technique is based on adhesion of pre-grown, freestanding crystals to a thermally oxidized $\mathrm{Si}$ wafer on which source and drain electrodes are deposited in advance. This process preserves the quality of the starting crystals, since it eliminates the need for deposition of metals and dielectrics directly onto the organic surface. We find that the mobility of the transistors fabricated in this way reaches room-temperature values of $0.4 \mathrm{~cm}^{2} / \mathrm{Vs}$ for holes in tetracene and $6 \mathrm{~cm}^{2} / \mathrm{Vs}$ for holes in rubrene. The observation of a non-monotonous temperature dependence of the mobility confirms the high quality of single-crystal devices. Moreover, a high level of reproducibility typifies the device characteristics, as well as good agreement with properties of single-crystal FETs fabricated using other techniques.

On account of the high-quality FET fabrication, we report the first observation of ambipolar transport in FETs fabricated on single crystals of copperand iron-phthalocyanine, using gold as a high work-function metal for the source and drain electrodes. In these devices, the room-temperature mobility of holes reaches $0.3 \mathrm{~cm}^{2} / \mathrm{Vs}$ in both materials. The highest mobility for electrons, observed in iron-phthalocyanines, is approximately one order of magnitude lower.

The compatibility of the electrostatic bonding technique with different types of gate insulators allows to investigate the integration of high- $\epsilon$ dielectric materials, like $\mathrm{Ta}_{2} \mathrm{O}_{5}$, in the organic single-crystal FETs. A technological aspect, that is important for both fundamental research and applications, is the observation that a small leakage current through the gate insulator affects the stability of organic single-crystal FETs. We find that, irrespective of the specific organic molecule and dielectric used, leakage current flowing through the gate insulator results in an irreversible degradation of the single-crystal FET performance. The degradation occurs even when the leakage current is several orders of magnitude 
smaller than the source-drain current. The experimental data indicate that a stable and high-quality operation requires the leakage current to be smaller than $10^{-9} \mathrm{~A} / \mathrm{cm}^{2}$.

The combination of the high quality and the high reproducibility of the FET measurements opens new opportunities for investigations. In particular, in combination with the integration of different types of gate insulators, it allows to study how aspects of the dielectric influence transistor operation, and moreover, it makes new operation regimes, like high charge densities, accessible for research.

We have demonstrated that the gate insulator plays a fundamental role in determining the device performance. A comparative study of rubrene singlecrystal FETs, fabricated using different materials as gate insulator, shows that the mobility of the charge carriers systematically decreases with increasing $\epsilon$ of the dielectric gate insulator: $\mu \propto \epsilon^{-1}$. This finding illustrates that the mobility of carriers in organic single-crystal FETs is an intrinsic property of the crystal/dielectric interface and that it does not only depend on the specific molecule used. We propose a mechanism of enhanced polaronic dressing of the charge carriers by the presence of a gate insulator with a large dielectric constant, that provokes a reduction of the mobility.

Additionally, we have investigated hole transport in a rubrene FET at densities up to $\sim 0.2$ holes/molecule, by use of a $\mathrm{Ta}_{2} \mathrm{O}_{5}$ gate dielectric. Close to the highest applied gate fields, i.e. for $0.1-0.2$ holes per molecule, we observe an unconventional reversible saturation, or decrease, of the channel conductivity. A comparison with other organic molecular FET measurements is insufficient to explain the distinct behavior in this, so far, unexplored operation regime. In this Thesis, we have discussed possible scenarios for the unconventional results, of which the influence of long-range Coulomb interactions provides a physically reasonable explanation, owing to its simplicity of arguments.

In short, in this work we have achieved successful fabrication of high-quality single-crystal FETs, exhibiting high mobilities and signs of intrinsic transport. Herewith, we have identified new aspects that influence charge transport in organic semiconductor FETs, and we have performed exploratory measurements in the charge density regime approaching one carrier per molecule. 


\section{Samenvatting}

\section{Organische één-kristal veld-effect transistoren Ruth W. I. de Boer}

De organische elektronica vormt een innovatief vakgebied, met interessante toe-passingen die complementair zijn aan de silicium halfgeleider technologie. Van- uit wetenschappelijk oogpunt is er grote interesse in het fundamentele begrip van elektrisch transport in organische halfgeleiders. Een goed ontwikkelde microscopische beschrijving ontbreekt echter nog steeds, wat te wijden is aan het gecompliceerde karakter van de veel-deeltjes polaronische ladingsdragers in moleculaire materialen.

Dit Proefschrift is het eerste waarin de intrinsieke ladingstransport-eigenschappen van organische halfgeleiders experimenteel bestudeerd zijn door gebruik te maken van organische één-kristal Veld-Effect Transistoren (FETs). Het elektrische veldeffect wordt veel gebruikt om dunne films van organische materialen te onderzoeken. Helaas zijn dunne-film transistoren niet geschikt voor het bestuderen van intrinsieke elektronische eigenschappen van organische geleiders, omdat hun karakteristieken vaak sterk beïnvloed worden door imperfecties in de filmstructuur en door onvoldoende zuiverheid van organische stoffen. Vanwege een betere moleculaire ordening en een hogere FET kwaliteit, fabriceren wij transistoren aan het oppervlak van een vrijstaand één-kristal van organische moleculen.

Ons werk begon met de karakterisering van gegroeide één-kristallen, door systematisch onderzoek van elektrisch transport in tetraceen één-kristallen met behulp van RuimteLadings-Gelimiteerde Stroom (SCLC) spectroscopie en VluchtTijd (TOF) metingen. Beide typen DC transportmetingen tonen aan dat de effectieve gatenmobiliteit in tetraceen bij kamertemperatuur waarden bereikt van bijna $\mu \simeq 1 \mathrm{~cm}^{2} / \mathrm{Vs}$ en ze laten zien dat de mobiliteit, binnen een bepaald temperatuurbereik, stijgt bij dalende temperatuur. Deze laatste observatie wordt over het algemeen beschouwd als een signatuur van de intrinsieke elektrische eigenschappen en de waarneembaarheid ervan in de transportmetingen demonstreert de hoge kwaliteit van de kristallen.

In een meer gedetailleerde studie van SCLC metingen en theorie hebben we aangetoond dat diepe ladingsvallen aan het oppervlak van een halfgeleidend mate- 
riaal, onder de elektrische contacten die gebruikt worden om lading te injecteren, een essentieel ingrediënt vormen voor een degelijk begrip van SCLC $I-V$ curven, dat voorheen genegeerd werd. Het belangrijkste effect van lading die gevangen is aan het oppervlak, is het modificeren van het elektrostatische profiel in de gehele bulk van het materiaal, wat het SCLC gedrag wezenlijk beïnvloed. Met behulp van simulaties van SCLC $I-V$ curven, waarin de aanwezigheid van ladingsvallen aan het oppervlak verwerkt is, kunnen we experimentele observaties reproduceren die niet verklaard worden met de conventionele theorie, waarin alleen ladingsvallen in de bulk van het materiaal in beschouwing worden genomen.

De belangrijkste oorzaak van ladingvallen aan het oppervlak van organische één-kristallen is schade die geïnduceerd wordt tijdens de depositie van elektrische contacten, wat aangeeft dat de depositie van materialen op het oppervlak van een organisch kristal een delicaat proces is. Dit vormt een niet-geringe complicatie voor het fabriceren van kwalitatief hoogstaande veldeffect transistoren op organisch één-kristallen. Daarom is de in dit Proefschrift beschreven ontwikkeling van de elektrostatische bindingstechniek voor het produceren van organische één-kristal FETs een belangrijke prestatie.

De elektrostatische bindingstechniek is gebaseerd op adhesie van vooraf gegroeide, vrijstaande kristallen aan een thermisch geoxideerde Si plak, waarop de source- (voor aanvoer van ladingdragers) en drain- (voor afvoer van ladingsdragers) elektroden vooraf aangebracht zijn. Tijdens dit proces blijft de oorspronkelijke kwaliteit van de kristallen behouden, omdat het de noodzaak elimineert om metalen en diëlektrica direct op het organisch oppervlak te deponeren. In transistoren die op deze manier gefabriceerd worden, meten we bij kamertemperatuur mobiliteiten tot $0.4 \mathrm{~cm}^{2} / \mathrm{Vs}$ voor gaten in tetraceen en $6 \mathrm{~cm}^{2} / \mathrm{Vs}$ voor gaten in rubreen. De observatie van een niet-monotone temperatuursafhankelijkheid van de mobiliteit bevestigt dat de kwaliteit van de één-kristal transistoren hoog is. Bovendien vertonen de transistorkarakteristieken een hoge mate van reproduceerbaarheid, en ook een grote overeenkomst met eigenschappen van één-kristal FETs die op andere manieren geproduceerd zijn.

Dankzij de kwalitatief hoogstaande FET fabricage melden wij de eerste observatie van ambipolair transport in FETs op één-kristallen van koper- en ijzerphthalocyanine, waarbij goud gebruikt is als metaal, met hoge werkfunctie, voor de source- en drain-elektroden. In beide materialen bereikt de gatenmobiliteit bij kamertemperatuur een waarde van $0.3 \mathrm{~cm}^{2} / \mathrm{Vs}$, en de hoogste elektronenmobiliteit, gemeten in ijzer-phthalocyanine, is bij benadering een orde van grootte lager.

De compatibiliteit van elektrostatische bindingstechniek met verschillende diëlektrische isolatielagen tussen de gate-elektrode en de source/drain-elektroden 
maakt het mogelijk om de integratie van materialen met een hoge diëlektrische constante $\epsilon$, zoals $\mathrm{Ta}_{2} \mathrm{O}_{5}$, in de organische FETs te onderzoeken. Een technologisch aspect, dat belangrijk is voor zowel fundamenteel onderzoek als voor toepassingen, is de observatie dat een kleine lekstroom door de isolatielaag de stabiliteit van de organische één-kristal FET aantast. We constateren dat lekstroom die door de isolator stroomt resulteert in een irreversibele degradatie van FET werking, onafhankelijk van welk organisch molecuul of diëlektricum gebruikt wordt. De degradatie treedt zelfs op als de lekstroom enkele ordes van grootte kleiner is dan de transportstroom tussen source- en drain-elektrode. De experimentele data duiden erop dat voor een stabiele en kwalitatief hoogstaande werking vereist is dat de lekstroom kleiner is dan $10^{-9} \mathrm{~A} / \mathrm{cm}^{2}$.

De combinatie van hoge kwaliteit en hoge reproduceerbaarheid van de FET metingen creëert nieuwe onderzoeksmogelijkheden. In het bijzonder maakt het mogelijk, in combinatie met de integratie van verschillende typen isolatielagen, om te bestuderen hoe aspecten van het diëlektricum de transistorwerking beïnvloeden. Bovendien maakt het nieuwe werkgebieden van de FET, zoals het regime van hoge ladingsdragersdichtheden, bereikbaar voor onderzoek.

We hebben aangetoond dat de isolator een fundamenteel bepalende rol speelt in de werking van de FET. Een vergelijkend onderzoek aan rubreen één-kristal FETs, geproduceerd met verschillende typen isolatielagen, laat zien dat de mobiliteit van de ladingsdragers systematisch daalt met groter wordende $\epsilon$ van het diëlektricum: $\mu \propto \epsilon^{-1}$. Deze bevinding illustreert dat de ladingsdragersmobiliteit in organische één-kristal FETs een intrinsieke eigenschap is van het raakvlak tussen het kristal en het diëlektricum, en dat het niet alleen afhangt van het specefieke molecuul dat gebruikt wordt. We opperen een mechanisme van versterkte polaronische omhulling van de ladingdragers door de aanwezigheid van een isolatiemateriaal met een grote diëlektrische constante, die een reductie van de mobiliteit veroorzaakt.

Daarnaast hebben we gatentransport in een rubreen FET onderzocht bij dichtheden tot $\sim 0.2$ gaten/molecuul, door gebruik te maken van een $\mathrm{Ta}_{2} \mathrm{O}_{5}$ isolatielaag. Bij de hoogste aangeboden elektrische velden, d.w.z. bij $0.1-0.2$ gaten per molecuul, nemen we waar dat de geleidbaarheid van het FET kanaal een onconventionele reversibele saturatie, of afname, vertoont. Een vergelijking met andere organische moleculaire FET metingen is onvoldoende om dit uitgesproken gedrag te verklaren, aangezien dit bereik van hoge ladingsdichtheden nog niet eerder onderzocht is. Van de verschillende scenario's die een mogelijke verklaring voor de onconventionele resultaten vormen en die we in dit Proefschrift besproken hebben, is de invloed van Coulomb interacties over lange afstanden een fysisch redelijke verklaring, dankzij de eenvoud van de gebruikte argumenten. 
Samenvattend hebben we in dit werk bereikt dat we met succes één-kristal FETs van hoge kwaliteit kunnen fabriceren, die hoge mobiliteiten en tekenen van intrinsiek transport vertonen. Daarmee hebben we nieuwe aspecten geïdentificeerd die het ladingstransport in organische halfgeleider FETs beïnvloeden, en we hebben de eerste verkennende metingen uitgevoerd in het ladingsdragersbereik dat nadert naar één drager per molecuul. 


\title{
Curriculum Vitae
}

\author{
Ruth Wilhelmine Ireen DE BOER
}

29-11-1976 Born in Roermond, the Netherlands

1989-1995 Grammar school "B. C. Schöndeln", Roermond

1995 Participant in International Chemistry Olympiad 1995

Beijing, China

1995-2000 M.Sc. Applied Physics (cum laude)

Eindhoven University of Technology

1995-1996 "Propaedeuse" (= first year) Applied Mathematics

Eindhoven University of Technology

1996-1998 "Propaedeuse" Chemical Engineering

Eindhoven University of Technology

1999 CERN Summer Student Programme

European Organization for Nuclear Research, Switzerland

2000 M.Sc. research: Electron Spectroscopy on $C_{60}$ and OPV5

(for the degree M.Sc. Applied Physics)

Solid State Physics, University of Groningen

Supervisors: Prof. Dr. G. A. Sawatzky and Prof. Dr. J. H. Wolter

2001-2005 Ph.D. research: Organic Single-Crystal Field-Effect Transistors

Kavli Institute of Nanoscience, Delft University of Technology

Promotor: Prof. Dr. ir. T. M. Klapwijk

Co-promotor: Dr. A. F. Morpurgo 


\section{List of publications}

\section{Journals}

1. Influence of surface traps on space-charge limited current R. W. I. de Boer, and A. F. Morpurgo, Phys. Rev. B 72, 073207 (2005)

2. Ambipolar $\mathrm{Cu}$ - and $\mathrm{Fe}$-Phthalocyanine single-crystal field-effect transistors R. W. I. de Boer, A. F. Stassen, M. F. Craciun, C. L. Mulder, A. Molinari, S. Rogge, and A. F. Morpurgo, Appl. Phys. Lett. 86, 262109 (2005)

3. Influence of the gate leakage current on the stability of organic single-crystal field-effect transistors

R. W. I. de Boer, N. N. Iosad, A. F. Stassen, T. M. Klapwijk, and A. F. Morpurgo, Appl. Phys. Lett. 86, 032103 (2004)

4. Organic single-crystal field-effect transistors

R. W. I. de Boer, M. E. Gershenson, A. F. Morpurgo, and V. Podzorov, Phys. Stat. Sol. A 201, 1302 (2004)

5. Influence of the gate dielectric on the mobility of rubrene single-crystal field-effect transistors

A. F. Stassen, R. W. I. de Boer, N. N. Iosad, and A. F. Morpurgo, Appl. Phys. Lett. 85, 3899 (2004)

6. Space charge limited transport and time of flight measurements in tetracene single crystals: A comparative study

R. W. I. de Boer, M. Jochemsen, T. M. Klapwijk, A. F. Morpurgo, J. Niemax, A. K. Tripathi, and J. Pflaum, J. Appl. Phys. 95, 1196 (2004)

7. Field-effect transistors on tetracene single crystals

R. W. I. de Boer, T. M. Klapwijk, and A. F. Morpurgo, Appl. Phys. Lett. 83, $4345(2003)$

\section{Books}

1. Organic single-crystal field-effect transistors

R. W. I. de Boer, M. E. Gershenson, A. F. Morpurgo, and V. Podzorov, Chapter 14 in Physics of organic semiconductors (Wiley-VCH, Weinheim, 2005) 


\section{Acknowledgements}

Well then, here it is: my Ph.D. thesis, the result of four years of intense work. It has cost me blood, sweat and tears... quite literally actually. But without the help of a number of people, I would never have been able to reach this point. I would like to thank all these people, and that is precisely what these last few pages are meant for.

First of all, I would like to thank my co-promotor Alberto Morpurgo for supervising me during more than four years of Ph.D. research. Your Italian temperament can be inspiring, funny, and intimidating. I admire your broad knowledge of physics and your scientific thoroughness, and although you tried to teach me that as much as possible, I still have a long way to go. Fortunately, there is at least one subject in which I beat you, which is Dutch! I am equally grateful to my promotor Teun Klapwijk. Although my being a woman was regularly brought up, it was no reason for you to give me a milder treatment than "the guys", and I appreciate that. It was a tough learning process, and I was surely not always happy with it, but I gained a lot from it. And if you give a compliment, you give a good compliment: the champagne was a hit!

I acknowledge Prof. Michael Gershenson and Vitaly Podzorov for a very pleasant cooperation. Misha, your enthusiasm during your two visits to Delft was really inspiring, and I really enjoyed our discussions on organic single crystals as well as foreign countries, ranging from Belgium to Nepal, and from Russia to Peru. Additionally, I would like to thank the people from the Organic molecular crystals and thin films group of the Stuttgart University, where I spent a wonderful week in September 2002. Prof. Norbert Karl, you have taught me more than I thought was possible in just one week. Jens Niemax and Jens Pflaum, thank you for providing, besides scientific support, also an evening program: in the bar with a beer and live music.

Good help came also from closer to home: Eindhoven, Leiden and Utrecht. I want to show my gratitude to the faculty of Applied Physics of the Eindhoven University of Technology for offering support. In particular Wim de Jonge, for giving me some self-confidence in a period when I was lacking it - I really appre- 
ciate that. I render thanks to Jeroen van den Brink from the Leiden University for valuable discussion about, among other things, electron-electron interactions, which helped me greatly in writing Chapter 9. Moreover, I acknowledge H. Kooijman from the Utrecht University for X-ray diffraction measurements on phthalocyanine crystals, as discussed in Chapter 7 .

Absoluut onmisbaar in dit gedeelte van mijn proefschift zijn mijn medepromovendi Hon Tin en Frank! Met z'n drieen was het een stuk makkelijker dan alleen. Tino, als kamergenoten hebben we veel lief en leed gedeeld. Ik denk met plezier terug aan onze vele gesprekken over de meest uiteenlopende onderwerpen tijdens dim-sum middagen, filmavonden en meetweekenden. Op de vraag "Wat is de zin van het leven?" zijn uiteindelijk maar twee antwoorden mogelijk: "42" of "Dit is de auto van mijn moeder." En daarom Tino: xièxie!!! Frank, jij deed me regelmatig beseffen dat de wereld groter was dan alleen mijn promotieonderzoek, en daar ben ik je erg dankbaar voor! We moeten nog altijd een proposal schrijven over Rashba spin-orbit interactie in organische kristallen, veel geld binnenhalen en dan in Thailand op het strand gaan liggen. Daar kunnen we in alle rust, biertje erbij, een oplossing bedenken voor het luxe keuze-probleem van onze generatie (immers: more is more... toch?).

Mijn studenten wil ik bedanken: Marinus, Gerdien, Melvin, en Carlijn. Ieder van jullie had zo zijn/haar bijzondere voorliefde - voor het kraken van een Calvéhuis, voor psychologie en zang, voor de Engel of voor Puss in Boots en andere dieren. Ik heb veel van jullie geleerd, en ik hoop dat dat andersom ook zo is. Additionally, I am grateful to the other co-workers of the organic single-crystal FETs project: Arno, Iulian, Anna, Nick, and Cvetanka. Don't drink too many cocktails in Club MED, okay? Of course, I don't forget the old crew members of organic electronics: Eduard, Diederik, Jos and Hugh, you were my "teachers in organics". Diederik, alias Reinaert of Paultje, ik heb bewondering voor de manier waarop jij je promotie wist te combineren met een musical-carrière. Hugh, you showed to me that chemists are not that bad after all! I recall your excitement in the lab, the hiking trip in Italy and many good laughs.

Er zijn nog veel andere mensen uit mijn directe werkomgeving die belangrijk voor me zijn geweest. In het bijzonder Ilona (kriebelvogeltje, maar vooral kwebbelmaatje) en ook Gabri, Maurits, Ruurd, Sebastiaan ("50 Cent") en Steffi, Joost, Martin, en Merlijn. Ja ja, die legendarische studenten-drinkavondjes (gelukkig mochten promovendi ook mee) liepen altijd uit op enkele ziekmeldingen de dag erna en een hoop goeie herinneringen, bijvoorbeeld aan de roze pet! Carlijn wil ik nog even opnieuw noemen. We hebben een enorm leuk jaar samengewerkt (je had mij helemaal niet nodig!), en gedurende die tijd ben je ook een goeie vriendin geworden. Lowlands was geweldig, Alexander de grote tegenvaller, maar 
we hebben de mountaineering-DVD nog in het verschiet.

Ik ben Emile van der Drift en andere medewerkers van DIMES zeer erkentelijk voor hun technische bijstand. Ook wil ik de technische stafleden Jan, Ron, Ben en de medewerkers van glasverwerking bedanken voor assistentie. En het secretariaat: Maria, Monique, Margaret en Daphne - de dames die in een gang vol wetenschappers zorgen voor vrolijkheid, ontspanning en voor al die administratieve zaken waar fysici zich liever niet mee bemoeien. Bovendien gaat mijn dank uit naar alle overige NF- en MED-leden die ik nog niet heb genoemd voor hun ondersteuning.

Graag wil ik hier extra aandacht schenken aan een paar heel speciale, langdurige vriendschappen. Hanneke, jouw steun was in de afgelopen jaren onbetaalbaar! Al zat je helemaal in Wijchen, je leefde mee met al mijn ups en downs. Je bent een heel bijzondere, lieve meid en ik ben echt heel blij voor je dat je weer helemaal gezond bent. Lis en Oc, we spreken elkaar niet elke week, maar ik wil jullie bedanken omdat ik weet dat ik altijd op jullie kan rekenen. En dat is al zo vanaf het "baby journaal" op de verjaardag van meneer John!

Ik heb Eindhoven als stad en universiteit vaak hartstochtelijk verdedigd tegenover de Delftse fietsenmakers. Mijn "Eindhovense vrienden" Paul en Hanneke, Hans en Mieke, Jacomien en Marco en Stijn, Petra en Alfred, Sandra en Frank, Lindy en Dirk, Frank, Birgitt en Michiel, Bas en Jenny, Patrick, Marjolein, Willem en Asnha, en Joep: bedankt voor de ontspannende weekendjes weg, de succes-wenskaarten en e-mailtjes, en voor het aandragen van stellingen tijdens verjaardagsfeesten.

De lijst met namen lijkt voor een buitenstaander misschien onnodig lang, maar toch vind ik dat er nog een paar bij horen. Walter, Anniek en de kleine Olaf. Walter, ik ben bang dat ik onze weddenschap van vier jaar geleden nu toch echt definitief verloren heb. Het is echt hopeloos! Enkele QT-ers: Laurens, Frank, Jorden, Hubert, Floris, Jelle, Ronald en Floor, voor zeer geslaagde bbq's op mijn dakterras, borrelavonden in Veldhoven en squashpartijtjes. En niet te vergeten Margaret (gek mens, je bent een schat!), Annette, Joost (die Schneehexe viel goed!), Ariston Dames 2 (Jaimy, Hanneke, Nicole, Inge, Sasha, Marijke, Thea), Robert en Jennifer, Jasper (dokter Jasper to be).

En tenslotte een hele dikke en dankbare knuffel voor mijn familie. Pap en mam, Kirsten, Renee en Eelco, Guusje en Rinske, voor jullie heb ik niet veel woorden nodig: jullie zijn schatten! Jullie onvoorwaardelijke steun, interesse en oprecht advies was, en is, voor mij heel veel waard!

Ruth de Boer

Delft, 9 september 2005 

